\title{
Review of Searches for Higgs Bosons and Beyond the Standard Model Physics at the Tevatron
}

\author{
Arnaud Duperrin ${ }^{\mathrm{a}}$ \\ CPPM, IN2P3-CNRS, Université de la Méditerranée, F-13288 Marseille, France
}

May 22, 2008

\begin{abstract}
The energy frontier is currently at the Fermilab Tevatron accelerator, which collides protons and antiprotons at a center-of-mass energy of $1.96 \mathrm{TeV}$. The luminosity delivered to the $\mathrm{CDF}$ and $\mathrm{D} \varnothing$ experiments has now surpassed the $4 \mathrm{fb}^{-1}$. This paper reviews the most recent direct searches for Higgs bosons and beyond-the-standard-model (BSM) physics at the Tevatron. The results reported correspond to an integrated luminosity of up to $2.5 \mathrm{fb}^{-1}$ of Run II data collected by the two Collaborations. Searches covered include: the standard model (SM) Higgs boson (including sensitivity projections), the neutral Higgs bosons in the minimal supersymmetric extension of the standard model (MSSM), charged Higgs bosons and extended Higgs models, supersymmetric decays that conserve or violate $R$-parity, gauge-mediated supersymmetric breaking models, long-lived particles, leptoquarks, compositeness, extra gauge bosons, extra dimensions, and finally signaturebased searches. Given the excellent performance of the collider and the continued productivity of the experiments, the Tevatron physics potential looks promising for discovery with the coming larger data sets. In particular, evidence for the SM Higgs boson could be obtained if its mass is light or near $160 \mathrm{GeV}$. The observed (expected) upper limits are currently a factor of 3.7 (3.3) higher than the expected SM Higgs boson cross section at $m_{H}=115 \mathrm{GeV}$ and 1.1 (1.6) at $m_{H}=160 \mathrm{GeV}$ at $95 \%$ C.L.
\end{abstract}

PACS. 1 4.80.Bn, 14.80.Cp, 14.80.Ly, 12.60.Jv, 12.60.Cn, 12.60.Fr, 13.85.Rm

\section{Contents}

2 The Tevatron accelerator . . . . . . . . .

3 The CDF and DØ detectors . . . . . . . . . .

4 Standard model Higgs boson . . . . . . . . . . .

5 Higgs bosons in the MSSM . . . . . . . . . . 13

6 Extended Higgs models . . . . . . . . . . . . . . 19

7 Beyond the standard model . . . . . . . . . . . . 20

8 Charginos and neutralinos . . . . . . . . . . . 20

9 Squarks and gluinos . . . . . . . . . . . 22

10 Gauge mediated SUSY breaking . . . . . . . . . 25

$11 R$-parity violation . . . . . . . . . . . . . 25

12 Long-lived particles. . . . . . . . . . . . . . . . . . 27

13 Leptoquarks . . . . . . . . . . . . . . . . . . . . . . . . . . . .

14 Compositeness . . . . . . . . . . . . . 28

15 Extra gauge bosons . . . . . . . . . . . . . . . . . . . . . 29

16 Large extra dimensions . . . . . . . . . . 30

17 CDF signature-based searches . . . . . . . . . . . 32

18 Conclusion . . . . . . . . . . . . . . 32

19 Acknowledgments . . . . . . . . . . . . 34

\section{Introduction}

The standard model (SM) predicts experimental observables at the weak scale with high precision. De-

a e-mail: duperrin@cppm.in2p3.fr spite the great success of this model, the electroweak symmetry breaking mechanism by which weak vector bosons acquire non-zero masses remains unknown. The simplest mechanism involves the introduction of a complex doublet of scalar fields that generate particle masses via their mutual interactions leading to the so-called SM Higgs boson with an unpredicted mass [1]. Furthermore, the SM fails to explain, for instance, cosmological phenomena like the nature of dark matter in the universe 2. These outstanding issues are strong evidence for the presence of new physics beyond the standard model. Among the possible extensions of the standard model, supersymmetric (SUSY) models [3. 4 provide mechanisms allowing for the unification of the forces and a solution to the hierarchy problem. Particularly attractive are models that conserve $R$-parity, in which SUSY particles are produced in pairs and the lightest supersymmetric particle (LSP) is stable. In supergravity-inspired models (SUGRA) [5], the lightest neutralino $\tilde{\chi}_{1}^{0}$ arises as the natural LSP, which being neutral and weakly interacting could be responsible for the dark matter in the universe.

This paper reports recent experimental results of direct searches for the Higgs boson and beyond-thestandard-model (BSM) physics based on data collected by the CDF and D $\varnothing$ Collaborations at the Fermilab Tevatron collider. The dataset analyzed corresponds 


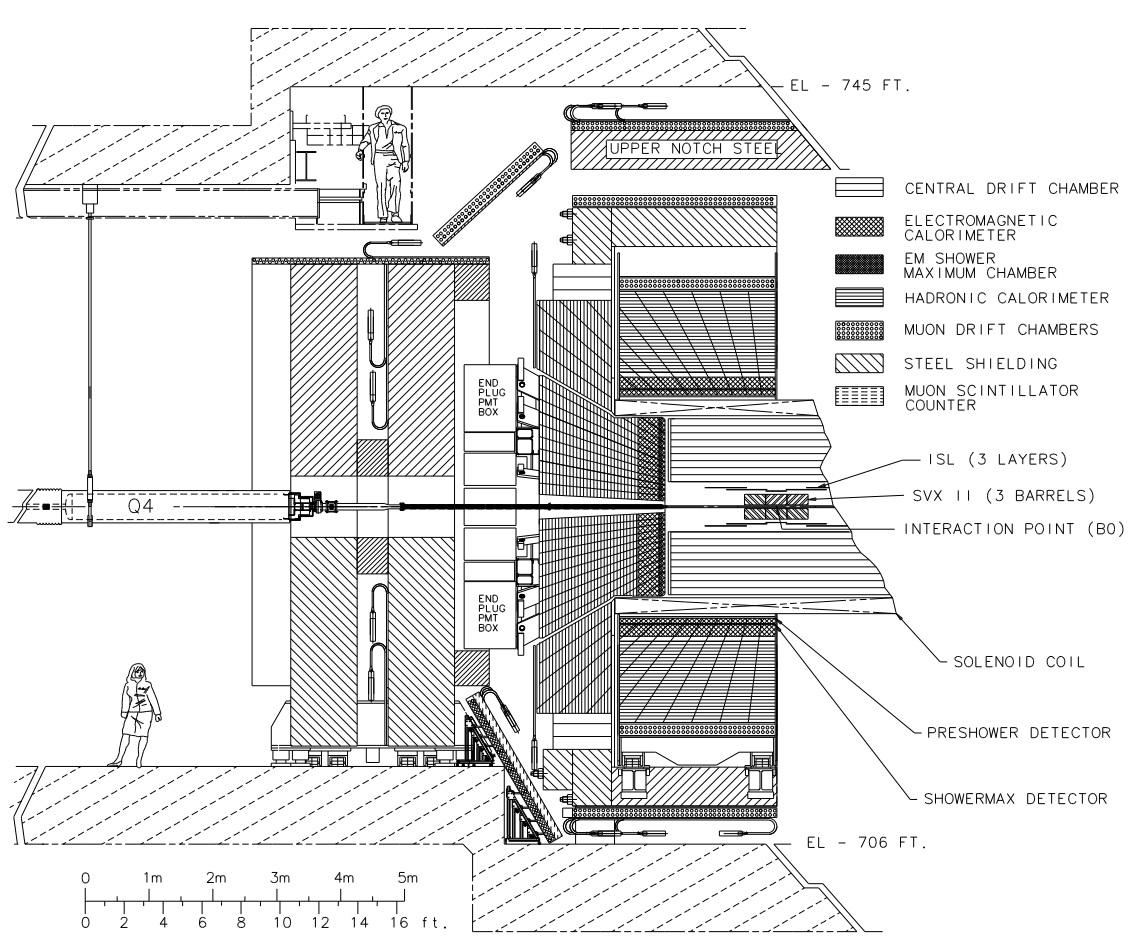

Fig. 1. Elevation view of the $\mathrm{CDF}$ detector in Run II 8 . to an integrated luminosity of up to $2.5 \mathrm{fb}^{-1}$. More details on the analyses can be found in Ref. 6, 7.

\section{The Tevatron accelerator}

The Tevatron is performing extremely well. For Run II, which started in March 2001, a series of improvements were made to the accelerator to operate at a centerof-mass energy of $1.96 \mathrm{TeV}$ with a bunch spacing of 396 ns. Before the 2007 shutdown, monthly integrated and peak luminosities of up to $167 \mathrm{pb}^{-1}$ and $2.6 \times 10^{32}$ $\mathrm{cm}^{-2} \mathrm{~s}^{-1}$, respectively, have been achieved. Since the most recent shutdown, beams with peak luminosity of $3.2 \times 10^{32} \mathrm{~cm}^{-2} \mathrm{~s}^{-1}$ have been delivered, with weekly integrated luminosity and antiproton hourly production rate reaching $58 \mathrm{pb}^{-1}$ and $26 \mathrm{~mA} /$ hours, respectively. The consequence, in terms of the number of interactions per crossing, is that the Tevatron is running in a mode similar to that expected at the Large Hadron Collider (LHC).

The DØ integrated luminosity delivered and recorded since the beginning of Run II is given in Table 1 (with similar values for $\mathrm{CDF}$ ).

Table 1. Run II luminosity delivered by the Tevatron accelerator, and luminosity recorded by the $\mathrm{D} \varnothing$ experiment.

\begin{tabular}{lll}
\hline & Delivered & Recorded \\
\hline Run IIa & $1.6 \mathrm{fb}^{-1}$ & $1.3 \mathrm{fb}^{-1}$ \\
Run IIb & $2.5 \mathrm{fb}^{-1}$ & $2.2 \mathrm{fb}^{-1}$ \\
\hline Total & $4.1 \mathrm{fb}^{-1}$ & $3.5 \mathrm{fb}^{-1}$ \\
\hline
\end{tabular}

\section{The CDF and D $\emptyset$ detectors}

A full description of the CDF (Fig. 1) and D $\varnothing$ (Fig. 2) Run II detectors in operation since 2001 is available in Ref. 8 9 . Both experiments are multipurpose detectors and are in a steady state of running. Detectors take data with an average efficiency of $85 \%$. An upgrade of the detectors to improve their performance for Run IIb was successfully concluded in 2006. The DØ upgrade included the challenging insertion of an additional layer close to the beam pipe of radiation-hard silicon (L0) to improve the tracking performance. CDF and $D \emptyset$ completed calorimeter and track trigger upgrades to significantly reduce the jet, missing energy, muon and electron trigger rates at high luminosity, while maintaining good efficiency for physics.

In the following, the $\mathrm{CDF}$ and $\mathrm{D} \varnothing$ detector components used in the analyses are briefly described. Both experiments use a cylindrical coordinate system around the proton beam axis in which $\theta$ and $\phi$ are the polar and azimuthal angles, respectively, and the pseudorapidity $\eta$ is defined as $\eta=-\ln [\tan (\theta / 2)]$. The transverse momenta and energy of a particle are defined as $p_{T}=p \sin \theta$ and $E_{T}=E \sin \theta$, respectively. In the following, imbalance in transverse momentum is referred to as missing transverse energy or $\mathbb{E}_{T}$. The trigger and data acquisition systems are designed to accommodate the high rates and large data volume of Run II. It comprise three levels of increasing complexity with a rate of accepted events written to permanent storage of about 50-150 Hz. The beam luminosity is determined by using counters located in the forward pseudorapidity region that measure the average number of inelastic $p \bar{p}$ collisions per bunch crossing. 


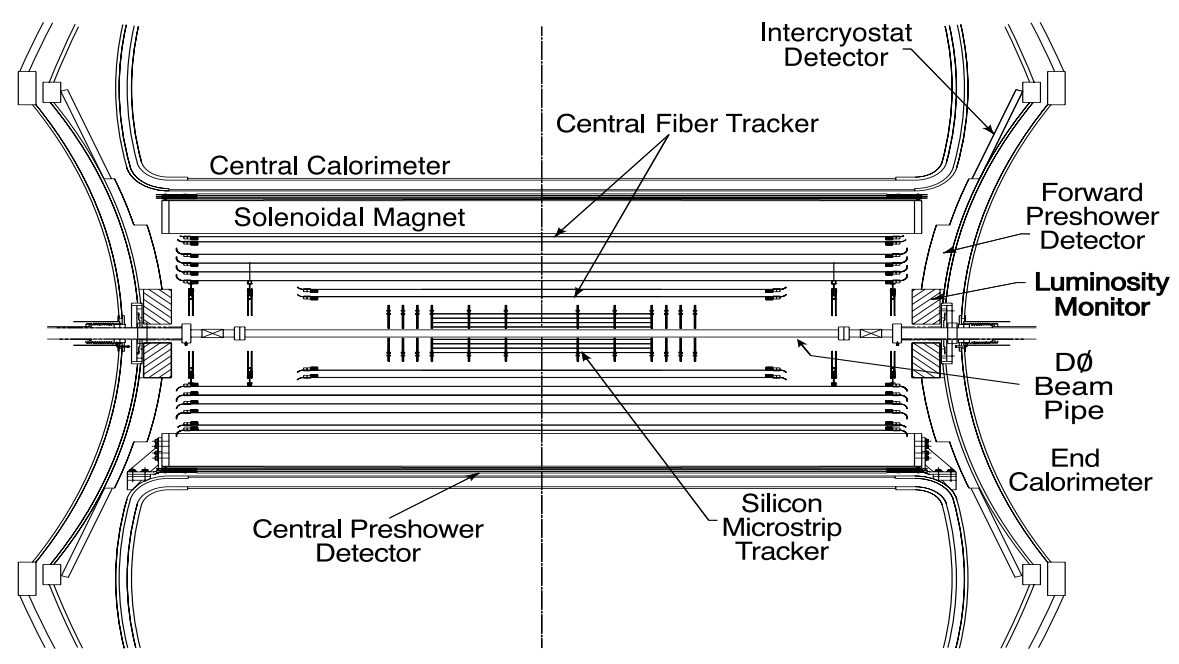

Fig. 2. Cross section of the central tracking system in the $x-z$ plane of the $\mathrm{D} \varnothing$ detector in Run II. Also shown are the locations of the solenoid, the preshower detectors, luminosity monitor, and the calorimeters 9 .

\subsection{CDF}

The tracking system consists of a cylindrical opencell drift chamber and silicon microstrip detectors in a 1.4 $\mathrm{T}$ magnetic field parallel to the beam axis. The silicon detectors [10] provide tracking information for $|\eta|<2$ and are used to detect collision and decay points. The drift chamber [11] surrounds the silicon detectors and covers the central rapidity region $|\eta|<1$. The energies of electrons and jets are measured in calorimeters covering the region $|\eta|<3.6$ and segmented into towers pointing toward the center of the detector. Jets are reconstructed from energy depositions in the calorimeter towers using a jet clustering cone algorithm [12] with a cone size of radius $\Delta R=$ $\sqrt{(\Delta \phi)^{2}+(\Delta \eta)^{2}}=0.4$. Corrections are applied to account for effects that can cause mismeasurements in the jet energy such as non-linear calorimeter response, multiple beam interactions, or displacement of the event vertex from the nominal $z=0$ position. Both the magnitude and direction of the $\mathscr{E}_{T}$ are recomputed after the jet energies have been corrected. Outside the calorimeters, layers of steel absorb the remaining hadrons leaving only muons, which are detected by drift chambers and scintillation counters up to $|\eta|<1.5$.

\section{$3.2 \mathrm{D} \emptyset$}

The central tracking system consists of a silicon microstrip tracker and a central fiber tracker, both located within a $1.9 \mathrm{~T}$ superconducting solenoid. A liquidargon and uranium calorimeter covers pseudorapidities up to $|\eta| \approx 4.2$. The calorimeter has three sections, housed in separate cryostats: the central one covers $|\eta|$ $\lesssim 1.1$, and the two end sections extend the coverage to larger $|\eta|$. The calorimeter is segmented in depth, with four electromagnetic layers followed by up to five hadronic layers. It is also segmented into projective towers of $0.1 \times 0.1$ size in $\eta-\phi$ space. An outer muon system, covering $|\eta|<2$, consists of a layer of tracking detectors and scintillation trigger counters positioned in front of $1.8 \mathrm{~T}$ toroids, followed by two similar layers after the toroids. Jet reconstruction is based on the Run II cone algorithm [13 with a cone size of 0.5 that uses energies deposited in calorimeter towers. Jet energies are calibrated using transverse momentum balance in photon+jet events. The missing transverse energy in an event is based on all calorimeter cells, and is corrected for the jet energy calibration and for reconstructed muons.

\section{Standard model Higgs boson}

The discovery of the Higgs boson is commonly considered to be the highest priority of particle physics today. This fundamental ingredient of the theory has not yet been observed and could be reachable at the Tevatron if the SM Higgs boson mass $m_{H}$ is light or near $160 \mathrm{GeV}$. The goal at the Tevatron is to find evidence for the Higgs boson using the full dataset, expected to correspond to about $7 \mathrm{fb}^{-1}$ by 2010 . A considerable effort has been devoted in recent years to improve the theoretical predictions and they are

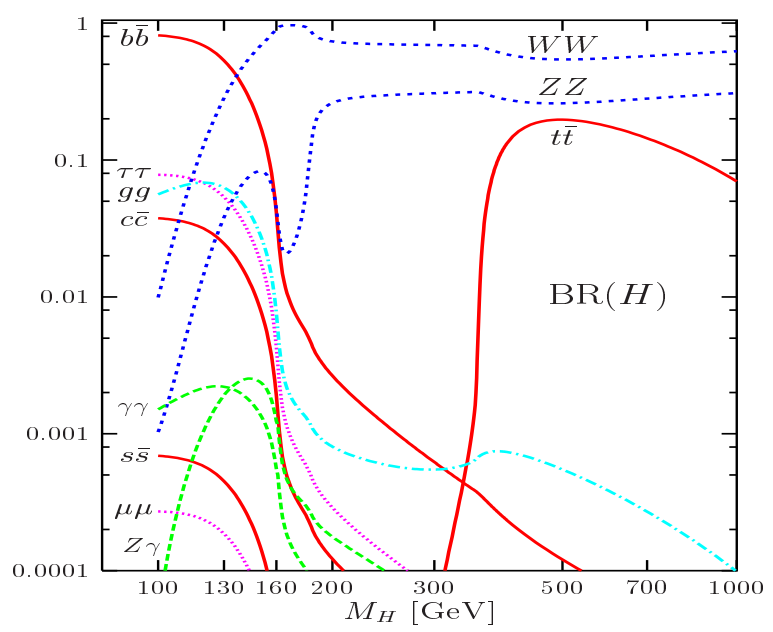

Fig. 3. Standard model Higgs boson branching fractions as a function of its mass [14]. 

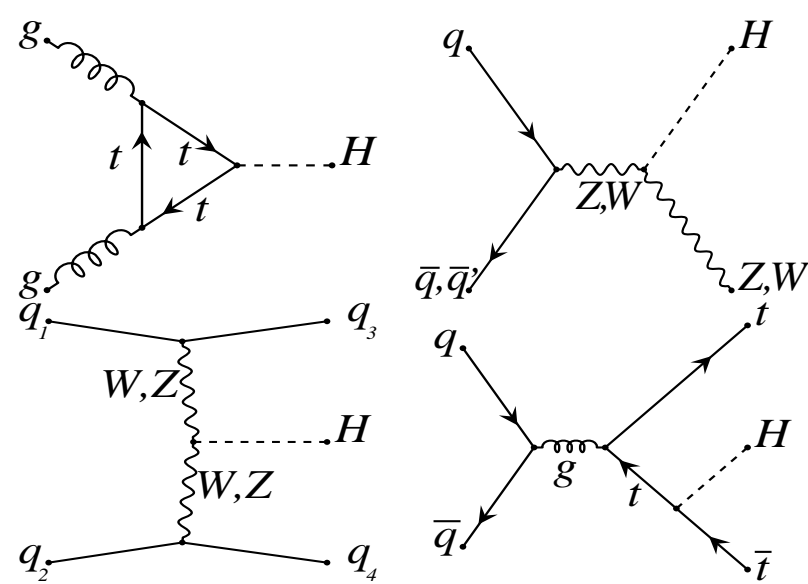

Fig. 4. Main diagrams for Higgs boson production at leading order: (top left) gluon fusion, (top right) Higgs associated production, (bottom left) vector boson fusion, (bottom right) associated production with heavy quark (top).

now known to good precision. The Higgs boson couples preferentially to the heaviest particles. As shown in Fig. 3, the decay mode $H \rightarrow b \bar{b}$ is the dominant one in the mass range $m_{H}<135 \mathrm{GeV}$, with a branching fraction $(\mathrm{Br})$ of $73 \%$ at $m_{H}=115 \mathrm{GeV}$. For Higgs masses above $\approx 135 \mathrm{GeV}$ the main decay mode is into $W W$ pairs, where one of the vector bosons is off-shell below the corresponding kinematic threshold.

The discovery of the Higgs boson is also among the main reasons for the construction of the Large Hadron Collider (LHC) at CERN, which is expected to begin operation in summer 2008. The LHC was designed such that the discovery of the SM Higgs boson, if it exists, would be guaranteed up to $\mathcal{O}(1 \mathrm{TeV})[15$, the highest energy consistent with general theoretical principles. Precision measurements, most notably of the top mass $m_{t}=172.6 \pm 1.4 \mathrm{GeV}[16$ at the Tevatron and of the $\mathrm{W}$ mass $m_{W}=80.398 \pm 0.025 \mathrm{GeV}$ [17,18 at LEP and at the Tevatron, however provide strong indications that the SM Higgs boson should be much lighter than that upper bound, having a mass smaller than $160 \mathrm{GeV}$ at $95 \%$ confidence level (C.L.) [19]. Direct searches for the Higgs boson at LEP in the $e^{+} e^{-} \rightarrow Z^{*} \rightarrow Z H$ reaction provide a lower limit of $114.4 \mathrm{GeV}$ [20, but also revealed several interesting candidate events with masses just above that lower bound. If the direct lower limit from LEP is taken into account to extract an upper bound from precision measurements, the SM Higgs boson mass upper limit becomes $190 \mathrm{GeV}$ at $95 \%$ C.L. Such a mass range is favorable to the Tevatrons reach.

\subsection{Higgs boson production}

The main diagrams for Higgs production are displayed at leading order in Fig. 4. The production cross section of the Higgs boson are summarized in Fig. [5] for $p \bar{p}$ collisions at the Tevatron. They are small, of the order of $0.1 \mathrm{pb}$, not including the decay branching fractions, while typical backgrounds such as $W+b \bar{b}$ or $Z+b \bar{b}$ have

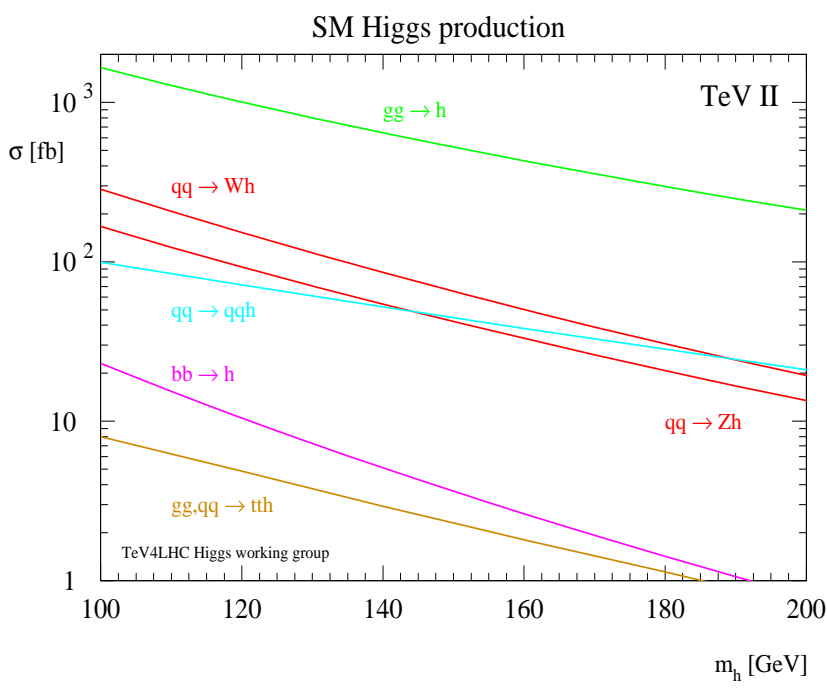

Fig. 5. Standard model Higgs boson production cross sections $(\mathrm{fb})$ at the Tevatron $(\sqrt{s}=1.96 \mathrm{TeV})$ for the most relevant production mechanisms as a function of the Higgs boson mass [21.

cross sections three orders of magnitude larger. The $q \bar{q} \rightarrow q q H$ vector boson fusion (VBF) process is known at next-to-leading order (NLO) in QCD and is marginal at the Tevatron with cross sections between $0.1-0.02 \mathrm{pb}$ for masses $100 \mathrm{GeV}<m_{H}<200 \mathrm{GeV}$ [22. The associated production process $t \bar{t} H$ is also known at NLO in QCD and can be exploited only at the LHC [23]. The bottom fusion $b b \rightarrow H$ process is known at next-tonext-to-leading order (NNLO) in QCD in the five-flavor scheme [24, 25] and has a cross section of $25 \mathrm{fb}$ for $m_{H} \approx 100 \mathrm{GeV}$. The two main production modes at the Tevatron are therefore gluon fusion $g g \rightarrow H+X$ and associated production $q \bar{q} \rightarrow W H+X, q \bar{q} \rightarrow Z H+X$. The associated production processes have cross sections known at NNLO in QCD and NLO for the electroweak corrections with a rather small residual theoretical uncertainty that is less than $5 \%$ 25,26. The gluon fusion process $g g \rightarrow H+X$ is known at NNLO in QCD (in the large top-mass limit) and at NLO in QCD for arbitrary top mass, with an overall residual theoretical uncertainty estimated to be around $10 \%[27$.

All Higgs signals are simulated using PYтніA [28, and CTEQ5L or CTEQ6L 29 leading-order (LO) parton distribution functions. The signal cross sections are normalized to next-to-next-to-leading order (NNLO) calculations [25, 27, and branching fractions from HDECAY 30.

\subsection{SM backgrounds}

The dominant backgrounds to Higgs analyses at low mass comprise $W / Z+$ jets, $t \bar{t}$, single top, and multijet (instrumental) events. The latter are sometimes referred to as "QCD background". At high mass, di-boson processes represent the main contribution to the backgrounds. 


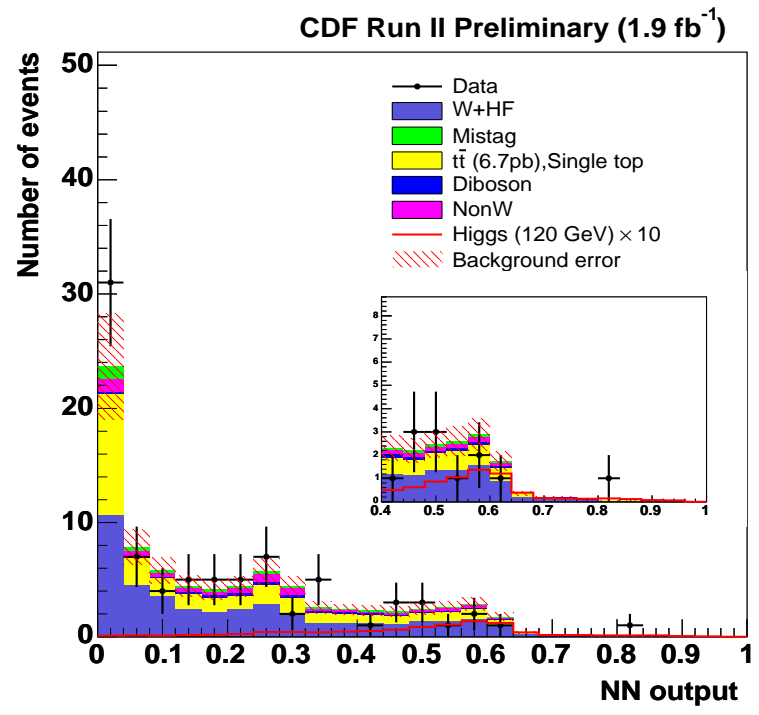

Fig. 6. Neural network output distribution obtained after final selection in the search for associated Higgs boson production ( $W H \rightarrow \ell \nu b \bar{b}$ channel) using $1.9 \mathrm{fb}^{-1}$ of CDF Run II data [42. Two of the jets in the event are required to be identified as containing b-quarks ( $b$-tagging). The background expectations and the observed data are shown. The expected Higgs signals, scaled as indicated, is represented for a neural network trained with a Higgs boson mass of $m_{H}=120 \mathrm{GeV}$. The insert shows a zoom-in the signal region.

For both CDF and $\mathrm{D} \varnothing$, the multijet background is estimated from data, usually in orthogonal samples to those used for the analyses. For CDF, backgrounds from other SM processes are generated using the PYTHIA, ALPGEN [31, MC@NLO 32] and HERWIG [33] programs. For D $\varnothing$, these backgrounds were generated using PYTHIA, ALPGEN and COMPHEP [34, with PYTHIA providing parton-showering and hadronization for all the generators. Background processes are normalized using either experimental data or next-to-leading order calculations from MCFM 35].

\subsection{Search strategy}

At the Tevatron, the most sensitive channels to search for a low mass SM Higgs boson $\left(m_{H}<135 \mathrm{GeV}\right)$ are those from associated production, with $W \rightarrow l \nu$ and $H \rightarrow b \bar{b}$, or $Z \rightarrow l l$ or $\nu \nu$ and $H \rightarrow b \bar{b}$. At high mass, $m_{H} \approx$ $160 \mathrm{GeV}$, the branching fraction is mainly into $W W$ boson pairs, and the leptonic decays of the $W$ are exploited through gluon-gluon fusion $p \bar{p} \rightarrow H \rightarrow W W^{(*)}$.

There are secondary modes that provide additional sensitivity. For intermediate masses around $135 \mathrm{GeV}$, all branching fractions are below $40 \%$; however, $p \vec{p}$ $\rightarrow W H \rightarrow W W W^{*} \rightarrow \ell^{ \pm} \ell^{ \pm}$and $p \bar{p} \rightarrow H \rightarrow \gamma \gamma$ are used to strengthen the discovery potential. At the Tevatron, the decay channel $p \bar{p} \rightarrow H \rightarrow Z Z^{(*)}$ does not significantly contribute due to combination of lower branching ratios for $H \rightarrow Z Z$ and $Z \rightarrow \ell \ell$ in addition to the acceptance for four leptons. Though both Collaborations

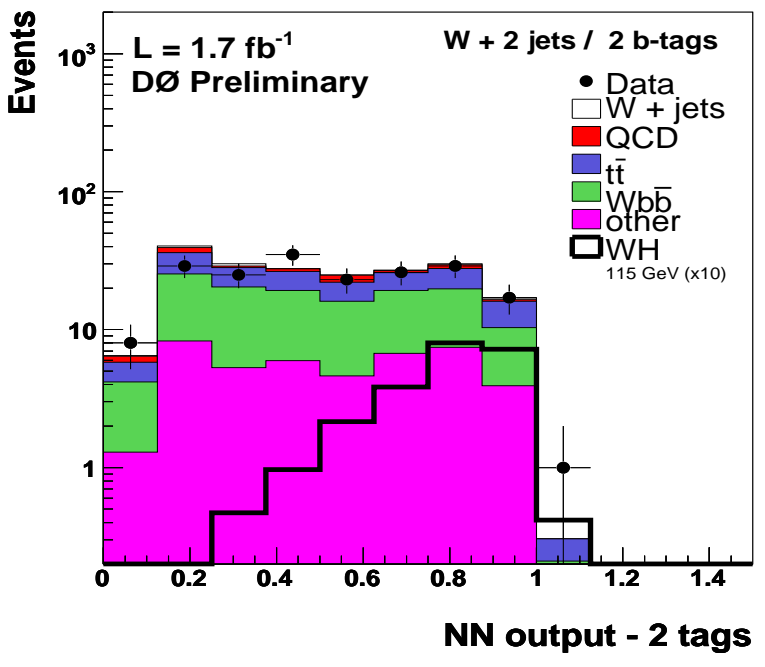

Fig. 7. $D \varnothing$ final analysis variable distribution (neural network output) used by the associated Higgs boson production search in the $W H \rightarrow \ell \nu b \bar{b}$ channel after requiring two $b$-tagged jets in the event 43 . The result is shown for an integrated luminosity of $1.7 \mathrm{fb}^{-1}$ of data. The background expectations and the observed data are shown. The expected Higgs signals, scaled as indicated, is represented for a neural network trained with a Higgs boson mass of $m_{H}=115 \mathrm{GeV}$.

do intend to add such analysis in the future, this mode has not yet been studied for the current combination. At low mass, a $5 \%$ contribution from the addition of the $H \rightarrow \tau \tau$ decay mode is included. The CDF experiment contributes to the most recent SM Higgs combination with this new analysis searching for the Higgs bosons decay to a tau lepton pair, in three production channels: gluon fusion, associated production, and VBF (details are given later).

\subsection{1 b-identification:}

At low mass, the searches primarily focus on the dominant $H \rightarrow b \bar{b}$ decay, which leads to the presence of $b$ jets for the signal. The sensitivity of the Higgs boson searches is significantly increased by using a lifetimebased heavy-flavor tagging algorithm ( $b$-tagging) which computes a probability for a jet to be light-flavored based on the impact parameters of the tracks in the jet. Another method is based on secondary vertex reconstruction.

For instance, D $\varnothing$ combines in a neural network (NN) discriminant 36, several kinematic variables sensitive to transversely-displaced jet vertices and jet tracks with large transverse impact parameters relative to the hard-scatter vertices. The NN is trained to identify heavy-flavor quark decays and reject jets arising from light-flavor quarks or gluons. By adjusting the minimum requirement on the $b$-tagging $\mathrm{NN}$ output, a spectrum of increasingly stringent $b$-tagging operating points is achieved, each with a different signal efficiency and purity. The analyses are usually separated 

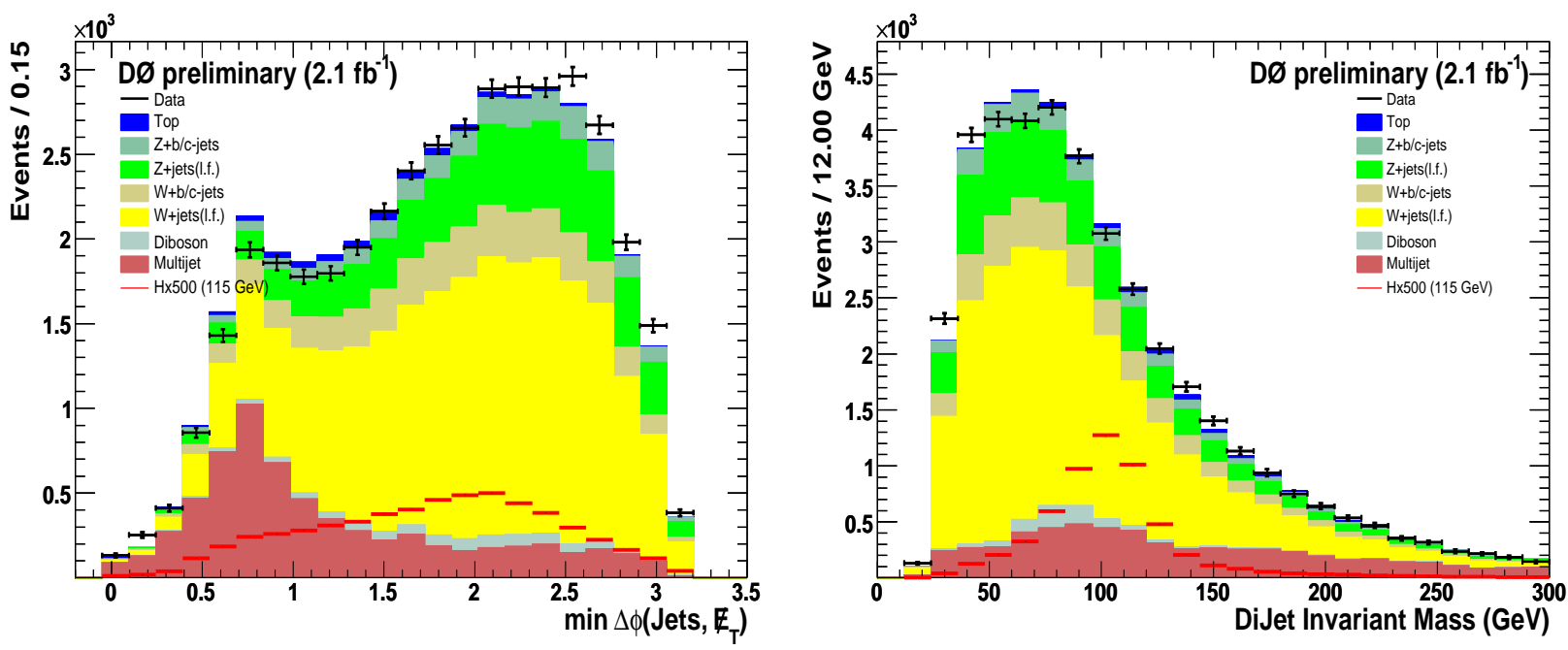

Fig. 8. Distributions of the $\Delta \phi_{\min }\left(\mathbb{E}_{T}\right.$,jets) (left), the minimum of the differences in azimuth between the direction of $E_{T}$ and the direction of any jet, and of the dijet invariant mass of the two leading jets (right), in the analysis sample before $b$-tagging for the $\mathrm{D} \varnothing$ search in the $p \bar{p} \rightarrow Z H \rightarrow \nu \bar{\nu} b \bar{b}$ channel using $2.1 \mathrm{fb}^{-1}$ of data [46. The various background contributions (SM and multijet) are shown. Distributions for a signal with a Higgs boson mass of $115 \mathrm{GeV}$ are also shown, scaled as indicated.

into those where two of the jets are $b$-tagged with a loose tagging requirement and those where only one jet is tagged with a tight requirement. Typically, the $b$-tagging tight (loose) operating point is selected such that, for jets with $p_{T}$ of $\approx 50 \mathrm{GeV}, 0.5 \%$ (1.5\%) of the light-flavored jets are tagged while the tagging efficiency for $b$-quark jets is $50 \%(60 \%)$.

\subsubsection{Advanced analysis techniques:}

After kinematic selection and $b$-tagging, the remaining backgrounds in a Higgs boson search would ideally be due solely to the associated production of a $W$ or $Z$ boson with a pair of $b$ quarks. The Higgs boson would then appear as a $b \bar{b}$ resonance over a broad continuum. The significance of a Higgs boson signal is therefore directly related to the mass resolution of a system of two $b$ quark jets, and thus to the jet energy resolution. The dijet mass is however not the only feature which allows discrimination between signal and background. Other, more subtle, differences in the kinematic properties of the signal and the backgrounds can be used. Because no single variable provides sufficient discriminating power, advanced analysis techniques have to be used, such as neural networks or decision trees 37 . The matrix element approach, which makes full use of the event properties at leading order and in which there is already experience in the CDF and $\mathrm{D} \varnothing \mathrm{Col}-$ laborations, can also be introduced to enhance the discriminating power of, for example, a neural network. The performance of even the most elaborate multivariate analysis, however, critically depends on an accurate understanding of the characteristics of the signal and background processes.

\subsubsection{Standard model background understanding:}

One of the major goals of the searches is to first carry out a detailed assessment of the generators used for the simulation of those processes, by confronting them with measurements, which are becoming increasingly more precise as the datasets grow.

Monte Carlo simulations, mostly based on fixedorder matrix elements, are used to extrapolate these measurements into the Higgs signal regions. In order to validate the whole analysis chain, the associated production of vector bosons, $W Z$ and $Z Z$, can be used. With $W Z \rightarrow \ell \nu b \bar{b}$ and $Z Z \rightarrow \ell \ell / \nu \nu b \bar{b}$, the final states are actually almost identical to those considered in the search for the Higgs boson, up to the mass difference between the $Z$ boson and the Higgs boson. Observation of these reactions will therefore be among the main goals of the coming year, and their detailed analysis will pave the way for a definitive calibration of the Higgs boson searches, with a caveat that $Z \rightarrow b \bar{b}$ branching fraction is a factor of 3 or less than the $H \rightarrow b \bar{b}$. The next-to-leading order (NLO) $Z Z$ cross section at the Tevatron is $1.4 \pm 0.1 \mathrm{pb}$ [35], an order of magnitude above some of the expected SM Higgs production cross sections. Additionally, this process forms an irreducible background to Higgs searches in the $Z H$ channel. For instance, the CDF Collaboration recently submitted for publication the first measurement at a hadron collider of the cross section for $Z$ boson pair production in the leptonic decay channels with a significance of 4.4 standard deviations based on $1.9 \mathrm{fb}^{-1}$ of data [38. The measured cross section is $\sigma(p \bar{p} \rightarrow Z Z)=$ $1.4_{-0.6}^{+0.7}$ (stat. + syst. $)$ pb. The D $\varnothing$ Collaboration also conducted such a measurement based on $2.2 \mathrm{fb}^{-1}$ of data. Using the final state decay $Z Z \rightarrow \ell \ell \nu \bar{\nu}$, D $\varnothing$ observes a signal with a $2.4 \sigma$ significance and measures a 


\section{Double Vertex Tag (Signal Region)}

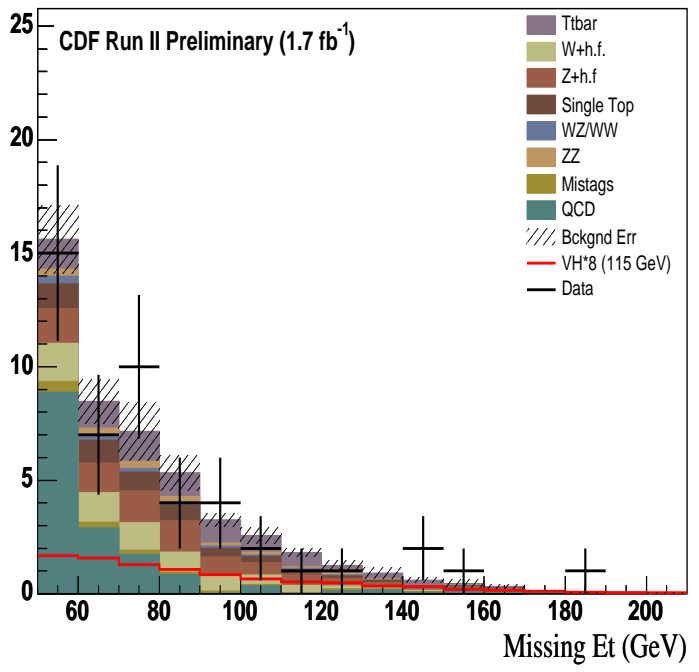

Double Vertex Tag (Signal Region)

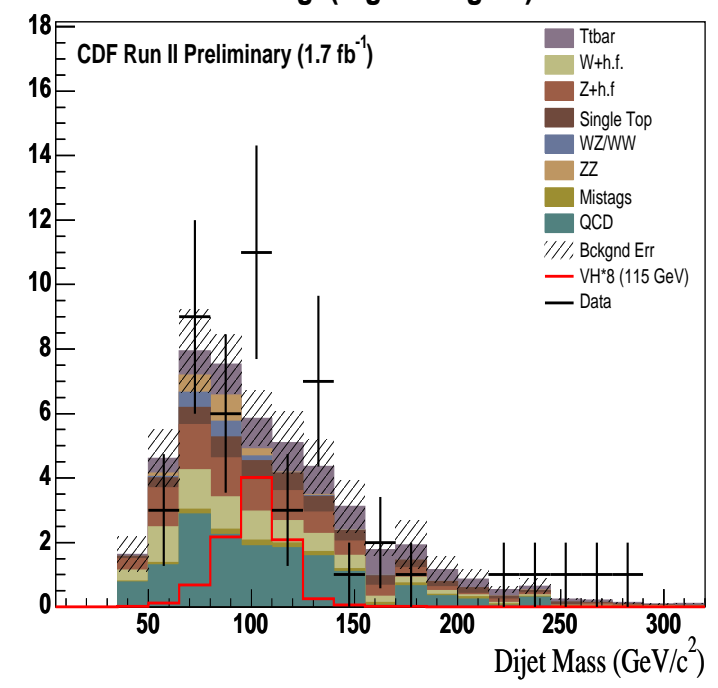

Fig. 9. Distributions of the $\mathbb{E}_{T}$ (top) and dijet invariant mass (bottom) in the signal region after requiring 2 $b$-tagged jets for the CDF search in the $p \bar{p} \rightarrow Z H \rightarrow \nu \bar{\nu} b \bar{b}$ channel using $1.7 \mathrm{fb}^{-1}$ of data 47]. The expected Higgs signal at $m_{H}=115 \mathrm{GeV}$ is scaled as indicated.

cross section $\sigma(p \bar{p} \rightarrow Z Z)=2.1 \pm 1.1$ (stat.) $\pm 0.4($ sys.) $\mathrm{pb}$ [39].

\subsection{Associated production}

Associated $V H$ production $(V=W, Z)$ can be distinguished from multijet and electroweak backgrounds by exploiting the leptons and/or the missing transverse energy in the final state.

\subsection{1 $p \bar{p} \rightarrow W H \rightarrow \ell \nu b \bar{b}:$}

The final state from $p \bar{p} \rightarrow W H$ production, where the $W$ boson decays leptonically, provides an ideal trigger signature to collect as many of the produced Higgs bosons as possible. The most recent published $W H$ searches have been performed with integrated luminosities of $955 \mathrm{pb}^{-1}$ for CDF [40 and $440 \mathrm{pb}^{-1}$ for $\mathrm{D} \varnothing$ [41. The latest updates with $1.9 \mathrm{fb}^{-1}$ from CDF 42 (winter 2008) and $1.7 \mathrm{fb}^{-1}$ from DØ 43. (summer 2007) use similar search strategies. The high- $p_{T}$ electron or muon is required to be isolated with $E_{T}$ (or $p_{T}$ ) greater than $20 \mathrm{GeV}$ and events with more than one isolated lepton are vetoed. Selected events must also display a significant missing transverse energy $\left(\mathbb{E}_{T}>20 \mathrm{GeV}\right)$. QCD events with false $W$ signatures are estimated with the data by computing the ratio of isolated to non-isolated leptons in a control region. Both $\mathrm{CDF}$ and $\mathrm{D} \varnothing$ use neural-network discriminants to separate the signal from the SM background events. As an illustration for these searches, Fig. 6] and Fig. 7 show some distributions of the final variables used by the CDF and $D \varnothing$ experiments for limit setting. The $D \varnothing$ search requires at least one tight $b$-tagged jet or exactly two loose $b$-tagged jets in the event, and two $b$-tagged jets are required in the CDF search. At $m_{H}=115 \mathrm{GeV}$, the ratio between the expected (observed) cross section limit and the SM value is 8.2 (7.3) for $\mathrm{CDF}$ and 9.1 (11.1) for $\mathrm{D} \varnothing$.

\subsection{2 $p \bar{p} \rightarrow Z H \rightarrow \nu \bar{\nu} b \bar{b}:$}

Triggering on $p \bar{p} \rightarrow Z H \rightarrow \nu \bar{\nu} b \bar{b}$ is more challenging than on the charged leptons from $W$ or $Z$ decays. This is because only hadronic jets are visible in the final state, which makes it difficult to distinguish it from standard multijet production via the strong interaction. The distinctive feature is the missing transverse energy carried by the neutrinos from the $Z$ decay. However, standard multijet events can also exhibit missing transverse energy due to fluctuations in the jet energy measurement by the calorimeter, or to semileptonic decay in heavy flavor jets. Complex topological triggers have therefore been designed, combining measurements of jet energies and directions, and missing transverse energy. The D $\varnothing$ Collaboration has published a result based on $260 \mathrm{pb}^{-1}$ of data [44 and the CDF Collaboration recently submitted for publication a search corresponding to an integrated luminosity of $1 \mathrm{fb}^{-1}$ [4]. Both experiments updated these results for the winter 2008 conferences with $2.1 \mathrm{fb}^{-1}$ [46] and $1.7 \mathrm{fb}^{-1}$ [4] of data for $\mathrm{D} \varnothing$ and $\mathrm{CDF}$, respectively.

One of the largest backgrounds in the $\mathbb{E}_{T}+$ jets channel involves heavy flavor multijet production. Although the probability for multijet events to create artificial missing energy at a significant level is small, the huge cross section of multijet production renders this background overwhelming at the initial stages of the analysis. Currently, severe selection criteria are used (mainly $E_{T}>50 \mathrm{GeV}$ ) in order to practically eliminate that background, thus introducing substantial inefficiencies. The normalization and shape of multijet events are obtained from the data to take into account all relevant instrumental effects and biases. This multijet control sample is extracted in the data sample 


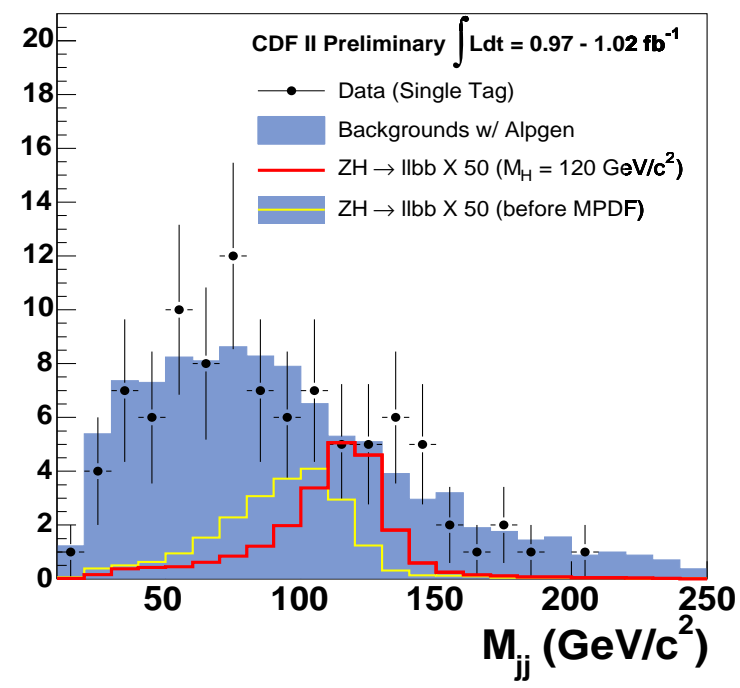

Fig. 10. Distributions of the dijet invariant mass in the signal region after requiring $2 b$-tagged jets for the CDF search in the $p \bar{p} \rightarrow Z H \rightarrow \ell \ell b \bar{b}$ channel using $\approx 1 \mathrm{fb}^{-1}$ of data [48. A correction (MPDF) is applied to reassign missing energy to the jets since the dominant source of $\mathbb{E}_{T}$ are jet energy mismeasurement. The expected Higgs signal at $m_{H}=115 \mathrm{GeV}$ is scaled as indicated.

where the $\mathbb{E}_{T}$ is aligned with the second jet. The distributions of the minimum of the differences in azimuth between the direction of the $\mathbb{E}_{T}$ and the direction of any jet, and the dijet invariant mass of the two leading jets, are shown in Fig. 8 for the $D \varnothing$ experiment. It is seen that the combination of the multijet and SM background provides a good description of the data in the pre-tag sample.

Advantage of the large branching fractions for $H \rightarrow$ $b \bar{b}$ is used by requiring the two leading jets to be $b$ tagged. Figure 9 shows the distributions of $\mathbb{E}_{T}$ and dijet invariant mass for the CDF analysis after all selection requirements are imposed. In the case of $W H \rightarrow$ $\ell \nu b \bar{b}$ production where the primary lepton from the $W$ boson decay falls outside of the detector acceptance and is not identified, the final state $W H \rightarrow \ell \nu b \bar{b}$ is the same as the $Z H \rightarrow \nu \bar{\nu} b \bar{b}$. The $W H \rightarrow \ell \nu b \bar{b}$ events contribute to significantly enhance the $\mathbb{E}_{T}+$ jets analysis sensitivity. Finally, a boosted decision tree technique was used for the $\mathrm{D} \varnothing$ search and a NN discriminant for the CDF search to calculate the cross section limit. For a $115 \mathrm{GeV}$ Higgs boson mass and requiring two $b$-tagged jets in the event, the observed/expected limits on the cross section of combined $Z H \rightarrow \nu \bar{\nu} b \bar{b}$ and $W H \rightarrow \ell \nu b \bar{b}$ production are 8/8.3 (7.5/8.4) times larger than the SM value for $\mathrm{CDF}(\mathrm{D} \varnothing)$.

\subsection{3 $p \bar{p} \rightarrow Z H \rightarrow \ell \ell b \bar{b}:$}

Searches for the Higgs boson from the process $p \bar{p} \rightarrow Z H$ $\rightarrow \ell \ell b \bar{b}$ in both $e^{+} e^{-}$and $\mu^{+} \mu^{-}$channels have been carried out by CDF [48] and Dø [4] in $1 \mathrm{fb}^{-1}$ of

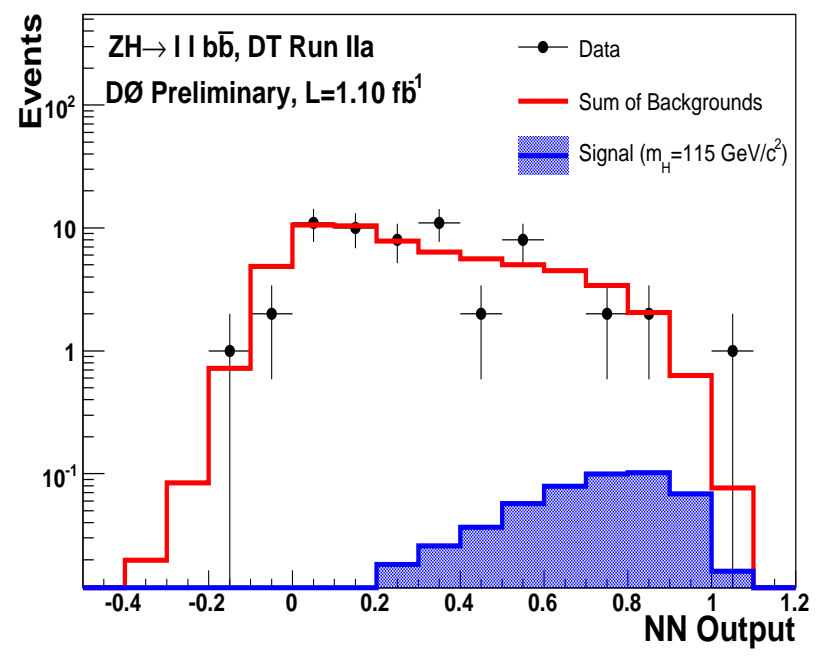

Fig. 11. Distributions of the neural net output variable after requiring $2 b$-tagged jets for the $\mathrm{D} \varnothing$ search in the $p \bar{p} \rightarrow Z H \rightarrow \ell \ell b \bar{b}$ channel using $1.1 \mathrm{fb}^{-1}$ of data 49 . The total predicted background, data and expected Higgs signal at $m_{H}=115 \mathrm{GeV}$ are shown.

data. The $\mathrm{D} \varnothing$ experiment has published a result based on $0.45 \mathrm{fb}^{-1}$ [50. These channels have a small background of mostly $Z+$ jets events due to the requirement of two leptons and a $Z$ mass constraint, but suffer from a smaller $Z$ branching fraction. To maintain signal efficiency and improve discrimination, the experiments employ neural networks trained to separate $Z H$ events from the main $Z+$ jets background and the kinematically different $t \bar{t}$ background. To improve sensitivity, the data are divided into single and double $b$ tagged channels (double $b$-tagged only for CDF); the results are shown in Fig. 10 and Fig. 11. The expected (observed) limits from the data as a ratio compared to the expected SM cross section are 20.4 (17.8) for the D0 analyses, and 16 (16) for the CDF search, at $m_{H}=115 \mathrm{GeV}$.

\subsection{4 $p \bar{p} \rightarrow W H \rightarrow W W W^{(*)} \rightarrow \ell^{ \pm} \ell^{ \pm}:$}

In the SM, the Higgs boson predominantly decays to a $W W^{(*)}$ pair for Higgs masses above $135 \mathrm{GeV}$. Although the scenario $W H \rightarrow W W W^{(*)} \rightarrow \ell^{ \pm} \ell^{ \pm}+X$ is not the most sensitive search for high masses, this process provides a unique experimental signature with two like-charge leptons from $W$ decays. Furthermore, in some scenarios with anomalous couplings, such as fermiophobic Higgs models, the branching fraction to $W W^{(*)}$ may be close to $100 \%$ for Higgs masses down to $\approx 100 \mathrm{GeV}[51,52,53$. The D $\varnothing$ Collaboration has searched for fermiophobic Higgs $\left(h_{f}\right)$ and published [54] results with $380 \mathrm{pb}^{-1}$ in the ee channel, $370 \mathrm{pb}^{-1}$ in the $e \mu$ channel, and $360 \mathrm{pb}^{-1}$ in the $\mu \mu$ channel, with the variations related primarily to different trigger requirements. Upper limits are set on $\sigma\left(p \bar{p} \rightarrow W h_{f}\right) \times$ $\operatorname{Br}\left(h_{f} \rightarrow W^{+} W^{-}\right)$between 3.2 and 2.8 pb for Higgs boson masses from 115 to $175 \mathrm{GeV}$ at $95 \%$ C.L. Recently, 

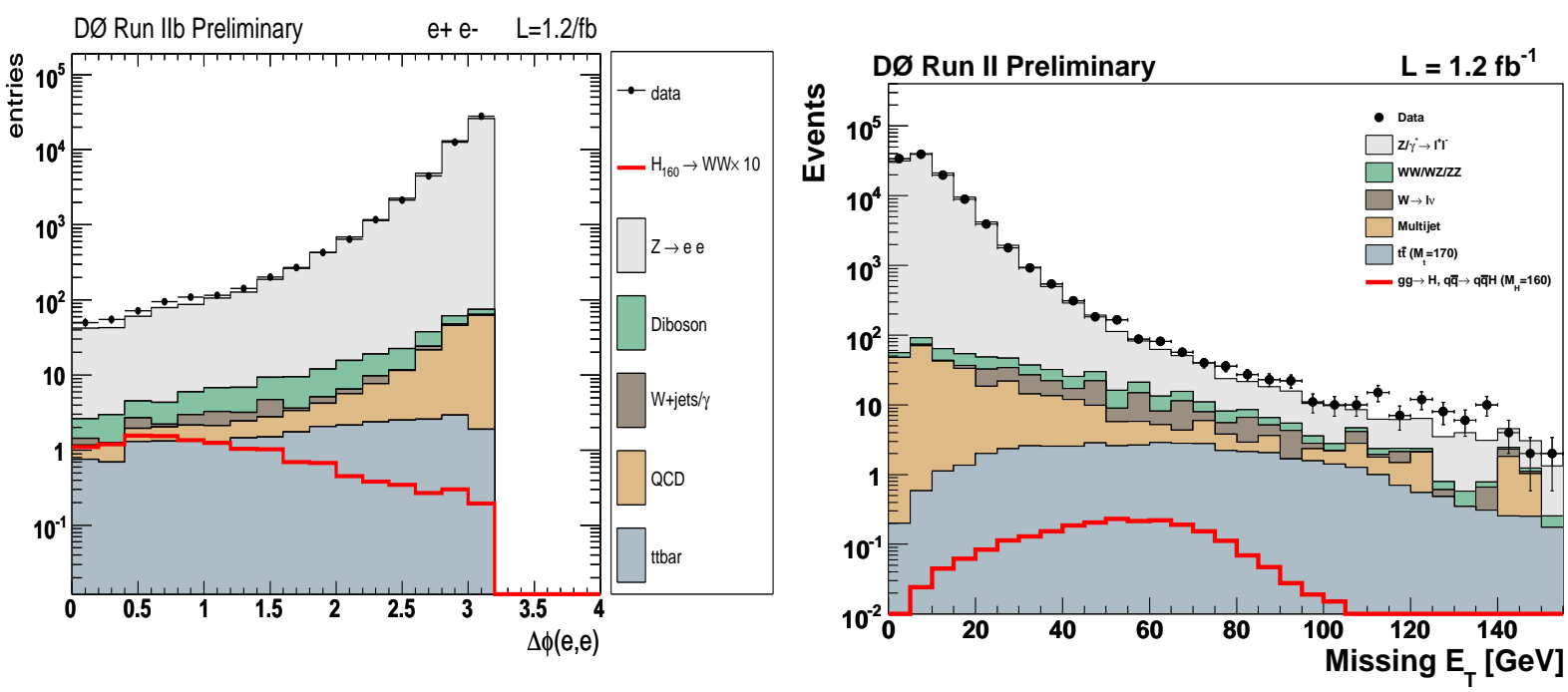

Fig. 12. Distributions of the opening angle $\Delta \phi_{e_{1} e_{2}}$ for the ee system (left) and $\mathbb{E}_{T}$ for the $\mu \mu$ system (right) at pre-selection level for the $\mathrm{D} \varnothing$ search in the $p \bar{p} \rightarrow H \rightarrow W W^{(*)} \rightarrow \ell \nu \ell^{\prime} \nu$ channel using $1.2 \mathrm{fb}^{-1}$ of data 61. The expected Higgs signal at $m_{H}=160 \mathrm{GeV}$, the predicted background, and the data events are shown.

Table 2. The numbers of signal events expected for a Higgs boson mass $m_{H}=160 \mathrm{GeV}$, events expected from SM backgrounds, and data events observed, for the CDF experiment using $2.4 \mathrm{fb}^{-1}$ of data [59. The SM Higgs boson production and decay are assumed to be $g g \rightarrow H \rightarrow W W^{*} \rightarrow l^{+} l^{-} \nu \nu$, where $l^{ \pm}=e, \mu$, or $\tau$. The final state $e$ trk $(\mu t r k)$ require an electron (a muon) and an additional track $(t r k)$.

\begin{tabular}{lccccccccrr}
\hline Category & $\begin{array}{c}\text { Higgs } \\
\left(m_{H}=160 \mathrm{GeV}\right)\end{array}$ & $W W$ & $W Z$ & $Z Z$ & $t \bar{t}$ & DY & $W \gamma$ & $W+$ jets & Total & Data \\
\hline$e$ e & 1.7 & 55.1 & 6.4 & 7.1 & 3.6 & 33.6 & 33.6 & 29.5 & $169 \pm 14$ & 171 \\
$e \mu$ & 3.8 & 131.7 & 3.9 & 0.4 & 8.7 & 27.4 & 29.4 & 34.1 & $235 \pm 21$ & 240 \\
$\mu \mu$ & 1.6 & 43.4 & 4.9 & 5.8 & 3.3 & 23.1 & 0.0 & 5.2 & $86 \pm 8$ & 83 \\
$e$ trk & 1.6 & 45.5 & 3.1 & 2.9 & 3.3 & 13.1 & 8.0 & 13.3 & $89 \pm 7$ & 107 \\
$\mu$ trk & 1.0 & 24.8 & 2.0 & 2.0 & 1.9 & 7.1 & 1.5 & 7.9 & $47 \pm 4$ & 60 \\
\hline Total & 9.5 & 300.3 & 20.6 & 18.2 & 20.8 & 104.0 & 72.3 & 90.0 & $626 \pm 54$ & 661 \\
\hline
\end{tabular}

CDF presented a preliminary result using $1.9 \mathrm{fb}^{-1}$ of data [55]. This search expects $0.46(0.19)$ event for a fermiophobic Higgs boson mass of 110 (160) GeV, assuming SM production cross section. The expected background is $3.23 \pm 0.69$ events, while 3 events are observed in the data. From these results, CDF sets limits of $2.2 \mathrm{pb}$ for $m_{h_{f}}=110 \mathrm{GeV}$ and $1.4 \mathrm{pb}$ for $m_{h_{f}}=160 \mathrm{GeV}$ at $95 \%$ C.L.

For these searches, the main physics background is $W Z \rightarrow \ell \nu \ell \ell$ production. The irreducible physics background, which comes from non-resonant $W W W$ triple vector boson production has a very low cross section (as does $t \bar{t}$ ). As the channel involves two neutrinos in the final state, the reconstruction of the Higgs mass is not feasible and the potential Higgs signal appears as an excess in the number of observed events with two like-charge leptons over the predicted SM background. In the absence of such an excess, upper cross section limits are set by the CDF [55] (DØ [56]) search with $1.9(1.1) \mathrm{fb}^{-1}$ of about $33(20)$ times above the SM Higgs boson cross section at $m_{H}=160 \mathrm{GeV}$.

\subsection{Gluon fusion}

The largest production cross section for the whole Higgs mass range of interest is the gluon fusion process due to the large top Yukawa couplings and the gluon densities. At the Tevatron, however, the gluon fusion process becomes relevant with a clear experimental signature only at high mass $\left(m_{H} \approx 160 \mathrm{GeV}\right)$, where the branching fraction is mainly into $W W$ boson pairs leading to a favorable final state with two leptons and two neutrinos. At low mass $\left(m_{H} \lesssim 135 \mathrm{GeV}\right)$, due to the large branching fraction of the Higgs boson into $b \bar{b}$, the gluon fusion Higgs production mode cannot be disentangled from the multijet background.

\section{$4.5 .1 p \bar{p} \rightarrow H \rightarrow W W^{(*)}:$}

At the Tevatron, the decay mode $p \bar{p} \rightarrow H \rightarrow W W^{(*)}$ provides the largest sensitivity for the SM Higgs boson search at a Higgs boson mass of $m_{H} \approx 160 \mathrm{GeV}$. Upper limits on the cross section times branching fraction in the leptonic decay modes $H \rightarrow W W^{*} \rightarrow \ell \ell^{\prime}\left(\ell, \ell^{\prime}=\right.$ 


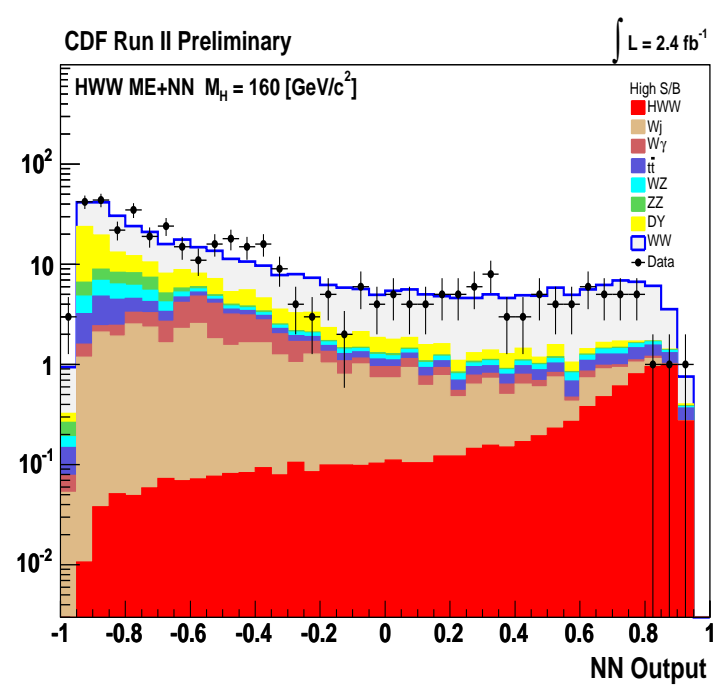

Fig. 13. Neural network template used as a final discriminant to search for the SM Higgs boson in the $p \bar{p} \rightarrow H \rightarrow W W^{(*)} \rightarrow \ell \nu \ell^{\prime} \nu$ decay channel using $2.4 \mathrm{fb}^{-1}$ of CDF data 59. The signal is shown for $m_{H}=160 \mathrm{GeV}$.

$e, \mu, \tau)$ from previous Run IIa data have already been published by the CDF (DØ) Collaboration with 360 $\mathrm{pb}^{-1}$ [57] $\left(325 \mathrm{pb}^{-1}[58]\right)$. The most recent data available from Run IIb have been recently analyzed and preliminary results have been presented by CDF 59 and $\mathrm{D} \varnothing 6061$ using $2.4 \mathrm{fb}^{-1}$ and $2.3 \mathrm{fb}^{-1}$ of data, respectively. In all final states, two isolated leptons of opposite sign originating from the same primary vertex are required. The background is dominated by $Z / \gamma^{*}$ and multijet events in which the leptons are typically back-to-back. It is therefore suppressed by requiring some missing transverse energy and with a cut on the opening angle $\Delta \phi_{\ell \ell}$ which is smaller for the signal than for the background due to the spin-correlation between the final state leptons in the decay of the spin-0 Higgs boson. Figure 12 shows the $\Delta \phi_{\ell \ell}$ and the $\mathbb{E}_{T}$ distributions at pre-selection level for the $\mathrm{D} \varnothing$ search.

After final selection, the CDF search finds 661 candidates with an expectation of $626 \pm 54$ background events and 9.5 signal events for a SM Higgs at $m_{H}=$ $160 \mathrm{GeV}$. Table 2 provides a detailed breakdown of the signal and background contributions in each final state. The presence of neutrinos in the final state prevents reconstruction of the Higgs boson mass. In order to maximize the signal sensitivity, a combined matrix element method and neural network approach are utilized to distinguish signal from background processes. An example of neural network template is shown in Fig. 13 for $m_{H}=160 \mathrm{GeV}$. The median expected $95 \%$ C.L. limit at a Higgs mass of $160 \mathrm{GeV}$ is $2.5_{-0.7}^{+1.0}$ times the SM prediction at NNLL, while the observed limit is 1.6 times the SM prediction. To improve the separation of signal from backgrounds, a neural network is also used for the $D \emptyset$ search in each of the three di-lepton channels. The $\mathrm{D} \varnothing$ expected and observed upper limits relative to the SM Higgs boson

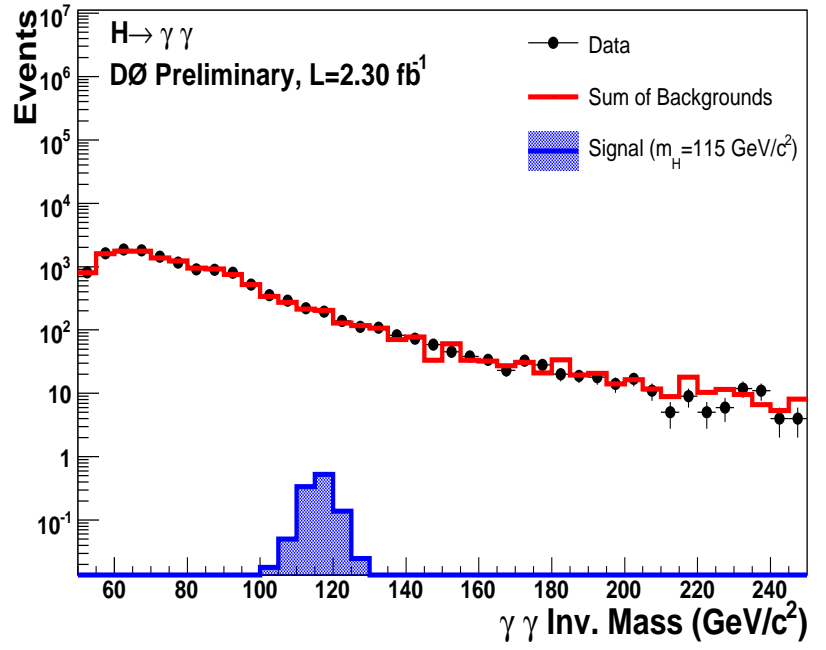

Fig. 14. Final variable distributions (diphoton invariant mass) for the $\mathrm{D} \varnothing$ Higgs search analysis in $p \bar{p} \rightarrow H \rightarrow \gamma \gamma$ final state using $2.3 \mathrm{fb}^{-1}$ of Run II data 63 . The total predicted background, observed number of event and expected Higgs signal at $m_{H}=115 \mathrm{GeV}$ are shown.

cross section prediction are 2.4 and 2.1 , respectively, for $m_{H}=160 \mathrm{GeV}$.

4.5.2 $p \bar{p} \rightarrow H \rightarrow \gamma \gamma:$

In the SM, the diphoton decay of the Higgs boson are suppressed at tree level and the branching fraction for this decay is $0.22 \%$ for a $130 \mathrm{GeV}$ Higgs boson mass. However, in some models beyond the SM, this decay can be enhanced significantly; some examples can be found in Refs. 51,52,53 and section 6 of this review. The DØ experiment has recently submitted for publication a search for a narrow resonance decaying into two photons using $1.1 \mathrm{fb}^{-1}$ of data 62. This result has been updated with $2.3 \mathrm{fb}^{-1} 63$ and the SM Higgs is used as a possible signal model to set upper limits on the production cross section times branching fraction $(H \rightarrow \gamma \gamma)$ for different assumed Higgs bosons masses. There are three major sources of background. The first source comes from Drell-Yan events where both electrons are misidentified as photons due to tracking inefficiencies, and is estimated with Monte Carlo simulations. The second source is from direct QED diphoton events and is also estimated using simulation. Finally, the background from $\gamma+$ jet and jet+jet events, where the jets are mis-identified as photons, is obtained from data.

The invariant mass of the two photon candidates in the interval $50 \mathrm{GeV}<m_{\gamma \gamma}<250 \mathrm{GeV}$, shown in Fig. 14, is used as input to the limit setting program. This search contributes to improve the global sensitivity in the difficult region around $m_{H} \approx 130 \mathrm{GeV}$, with a $\sigma \times B r$ ratio to SM of about 45 . 


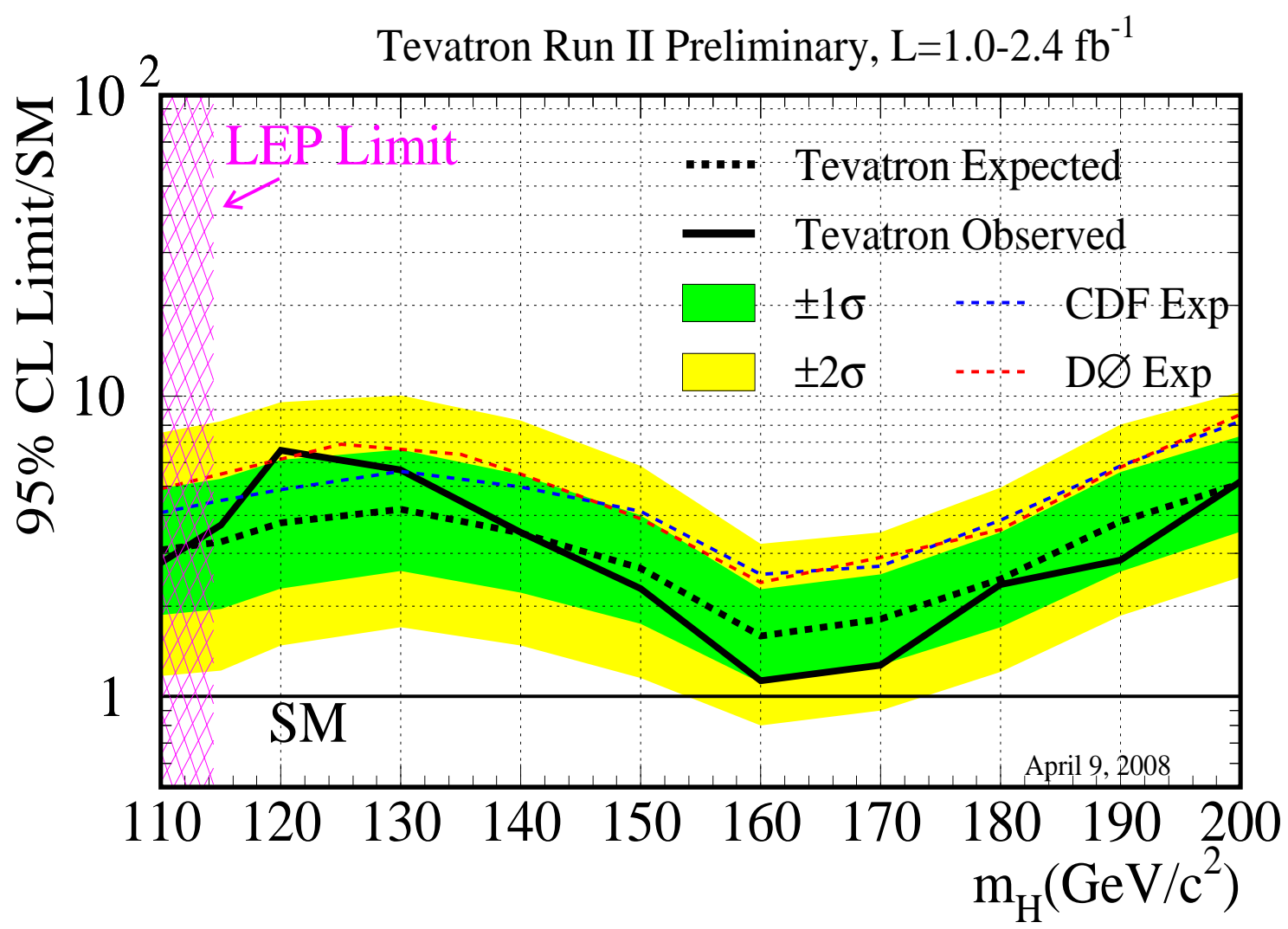

Fig. 15. The new Tevatron combination 66 presented at the winter 2008 conferences showing the upper bound on the SM Higgs boson cross section as a function of the Higgs boson mass. The contributing production processes include associated production $\left(W H \rightarrow \ell \nu b \bar{b}, Z H \rightarrow \ell \ell / \nu \nu b \bar{b}, W H \rightarrow W W^{+} W^{-}\right)$or gluon fusion $\left(H \rightarrow W^{+} W^{-}, H \rightarrow \gamma \gamma\right)$ or vector boson fusion, and $H \rightarrow \tau \tau$ produced in several modes. The limits at $95 \%$ confidence level (C.L.) are shown as a multiple of the SM cross section. The solid curve shows the observed upper bound, while the dashed curves show the expected upper bounds assuming no signal is present. Analyses are conducted with integrated luminosities ranging from $1.0 \mathrm{fb}^{-1}$ to $2.4 \mathrm{fb}^{-1}$ recorded by each experiment. The bands indicate the $68 \%$ and $95 \%$ probability regions where the limits can fluctuate in the absence of signal. The expected upper limits obtained from the CDF and D $\varnothing$ experiments are also shown. The region excluded by the LEP experiments is also shown 20].

\section{6 $H+X \rightarrow \tau^{+} \tau^{-}+2$ jets}

The CDF Collaboration recently conducted a search with about $2 \mathrm{fb}^{-1}$ of data using the $\tau$ decay mode of the Higgs boson 64. Several processes are considered: Higgs production in association with a vector boson $(W / Z)$, in which the vector boson decays into 2 jets ( $\mathrm{Br}$ are $67 / 70 \%$ for $W / Z$ ), vector boson fusion production in which the 2 jets coming from the proton and antiproton tend to have a large rapidity value and, finally, gluon fusion production. The analysis requires at least 2 jets, one hadronic tau with $p_{T}>15 \mathrm{GeV}$ and one leptonic tau identified as an isolated central electron (or muon) with $p_{T}>10 \mathrm{GeV}$. In this analysis, the final variable for setting limits is a combination of several neural network discriminants.
The expected (observed) sensitivity is roughly about $50 \%(30-40 \%)$ compared to that of searches for associated Higgs production. The expected cross section limit is 24 times the SM cross section for $m_{H}=$ $120 \mathrm{GeV}$. Combined with all other analyses, this result improves the global low mass Higgs search sensitivity.

\subsection{Combined upper limits}

All individual channels of both Tevatron experiments are combined to maximize the sensitivity to the Higgs boson. The combination of $\mathrm{D} \varnothing$ results based on 0.44 $\mathrm{fb}^{-1}$ of data has been published [41.

All new results included in this review have been used for the combination presented at the winter 2008 


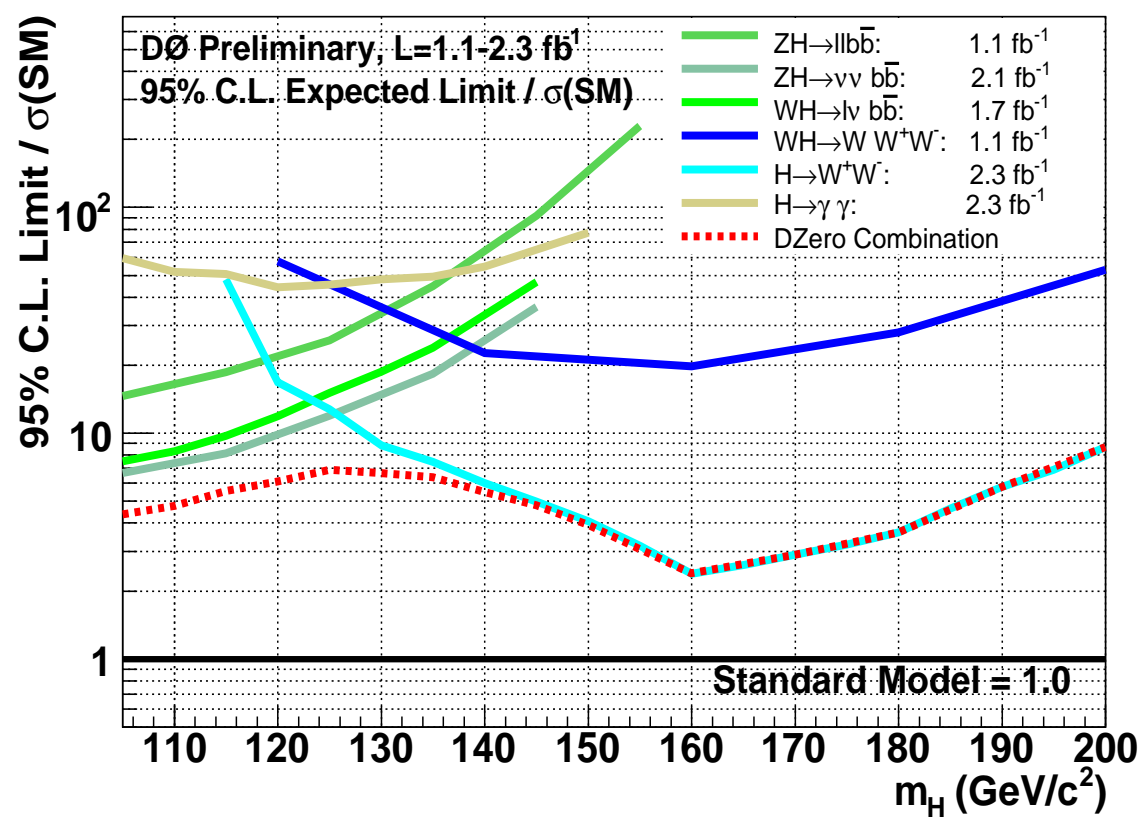

Fig. 16. Expected $95 \%$ C.L. cross section ratios for the individual $W H / Z H / H, H \rightarrow b \bar{b} / \gamma \gamma / W^{+} W^{-}$analyses in the $100 \mathrm{GeV}<m_{H}<200 \mathrm{GeV}$ mass range for $\mathrm{D} \varnothing$ alone using 1.1-2.3 $\mathrm{fb}^{-1}$ of data 68 .

conferences. These results have an improved sensitivity compared to the previous Tevatron combination presented in December 2007 65. The new Tevatron combination (21 April 2008, see Ref. 66]) includes all results from searches for a SM Higgs boson produced in association with vector bosons $(p \bar{p} \rightarrow W H \rightarrow \ell \nu b \bar{b}$, $p \bar{p} \rightarrow Z H \rightarrow \nu \bar{\nu} b \bar{b}, p \bar{p} \rightarrow Z H \rightarrow \ell \ell b \bar{b}$ and $p \bar{p} \rightarrow W H$ $\left.\rightarrow W W W^{*} \rightarrow \ell^{ \pm} \ell^{ \pm}\right)$or through gluon-gluon fusion $\left(p \bar{p} \rightarrow H \rightarrow W W^{(*)}\right.$ and $\left.p \bar{p} \rightarrow H \rightarrow \gamma \gamma\right)$ or vector boson fusion, and $H \rightarrow \tau \tau$ produced in several modes, in data corresponding to integrated luminosities ranging from $1.0-2.4 \mathrm{fb}^{-1}$ at CDF 67 and $1.1-2.3 \mathrm{fb}^{-1}$ at D $\varnothing 68$. This is the first time that searches for Higgs bosons decaying to two photons or two tau leptons are included in the combination.

\subsubsection{Method used for the combination}

To simplify their combination, the searches are separated into twenty nine mutually exclusive final states (thirteen for CDF, sixteen for DØ). In addition, several types of combinations are performed using the Bayesian 67] and Modified Frequentist 69] approaches to check that the final result does not depend on the details of the statistical method used for the combination. The Modified Frequentist approach is sometimes called the LEP $C L_{s}$ method, which is based on the log-likelihood ratio $(L L R)$ test statistic

$$
\begin{gathered}
\operatorname{LLR}(n)=-2 \ln (Q), \\
Q=L(s+b) / L(b)=\frac{e^{-(s+b)}(s+b)^{n}}{n !} / \frac{e^{-b}(b)^{n}}{n !},
\end{gathered}
$$

where $s$ and $b$ are the expected numbers of signal and background events while $n$ is the number of data events. For all channels, the $L L R$ values per bin are added in order to use the shape information of the final discriminating variable and to combine the different channels. In addition, the $C L_{s}$ method has been extended to involve a fit of the nuisance parameters for each event, which maximizes the sensitivity in the context of large background, small signals and large uncertainties [70. Both $C L_{s}$ and Bayesian methods use Poisson likelihoods and agree within $\sim 10 \%$. They rely on distributions of the final discriminants like NN output, matrix-element likelihoods, or dijet mass and not only on the event counting. The uncertainty on the expected number of events as well as the shape of the discriminant variables are included in the systematic uncertainties.

\subsubsection{Systematic uncertainties}

The correlations of systematic uncertainties between channels, rates and shapes, signals and backgrounds, within and between experiments are considered. Many sources are measured using data. Sometimes they are large but correspond to backgrounds with small contribution. Furthermore, the uncertainties are constrained by fits to the nuisance parameters and do not necessarily affect the result significantly.

The following dominant sources of systematic uncertainties are taken into account in deriving the final results. Each experiment has a luminosity uncertainty of $\approx 6 \%$, of which $\approx 4 \%$ is correlated. Depending on the channel, the range of uncertainties from SM cross sections used to normalize the simulation vary between $4 \%$ and $20 \%$. The uncertainty needed to describe heavy flavor $(\mathrm{HF})$ fractions in $W / Z+$ jets and the effect of the NLO HF cross section normalisation 
are evaluated to be $20 \%-50 \%$, depending on how the background is estimated, from data or simulation. The scale factor used to adjust the $b$-tagging efficiencies in the simulation results in $4 \%-25 \%$ uncertainties, depending on the number of tagged jets required. The uncertainty from the jet energy scale ranges between $1 \%$ and $20 \%$, depending on the background process. The uncertainties resulting from the modelling of QCD multijet background are dominated by statistics in the samples from which the estimates are derived and can reach $30 \%$. The uncertainties from lepton identification and reconstruction efficiencies ranges from negligible to $13 \%$. Finally, the uncertainty from the trigger efficiency is less than $5 \%$.

\subsubsection{Combined results}

The cross section limits on SM Higgs boson production $\sigma \times \operatorname{Br}(H \rightarrow X)$ obtained by combining $\mathrm{CDF}$ and $\mathrm{D} \varnothing$ results with up to $2.4 \mathrm{fb}^{-1}$ of data [66 are displayed in Fig. 15. The result is normalized to the SM cross section, where a value of one would indicate a Higgs mass excluded at 95\% C.L. The observed (expected) upper limits are a factor of $3.7(3.3)$ higher than the expected SM Higgs boson cross section at $m_{H}=115 \mathrm{GeV}$ and $1.1(1.6)$ at $m_{H}=160 \mathrm{GeV}$.

The combined $\mathrm{CDF}$ and $\mathrm{D} \varnothing$ result represents a $40 \%$ improvement in expected sensitivity over each single experiment. The observed (expected) limits on the SM ratios are 5.0 (4.5) for CDF and 6.4 (5.5) for $\mathrm{D} \varnothing$ at $m_{H}=115 \mathrm{GeV}$, and $1.6(2.6)$ for $\mathrm{CDF}$ and $2.2(2.4)$ for $\mathrm{D} \varnothing$ at $m_{H}=160 \mathrm{GeV}$. The sensitivity of each individual analysis from $\mathrm{D} \varnothing$ alone is given in Fig. 16 As shown in this figure, the associated production and gluon fusion processes play an important complementary role to improve the sensitivity for the intermediate mass region around $m_{H}=135 \mathrm{GeV}$, which is the most difficult mass to probe at Tevatron.

\subsection{SM Higgs boson prospects}

It is expected that the sensitivity needed to reach a $95 \%$ C.L. limit exclusion, at a mass $m_{H} \approx 160 \mathrm{GeV}$, will be reached by the end of 2008 by the combined Tevatron experiments.

Since the first CDF and DØ combination in 2006, a lot of progress has been made, resulting in better sensitivity in all channels (i.e., neural network $b$-tagger, improved selections, matrix-element techniques). Many of these improvements led to an equivalent gain of more than twice the luminosity, which means that the sensitivity has progressed faster than one would expect from the square root of the luminosity gained.

Recent projections in sensitivity have been made based on achievable improvements of the current analyses. These include progress on the existing improved lepton identification efficiency, heavy-flavor taggers and $b$-tagging enhancement from the DØ layer L0, upgraded trigger acceptance, increased usage of advanced analysis techniques, jet resolution optimization, reduced systematics, and inclusion of additional channels.

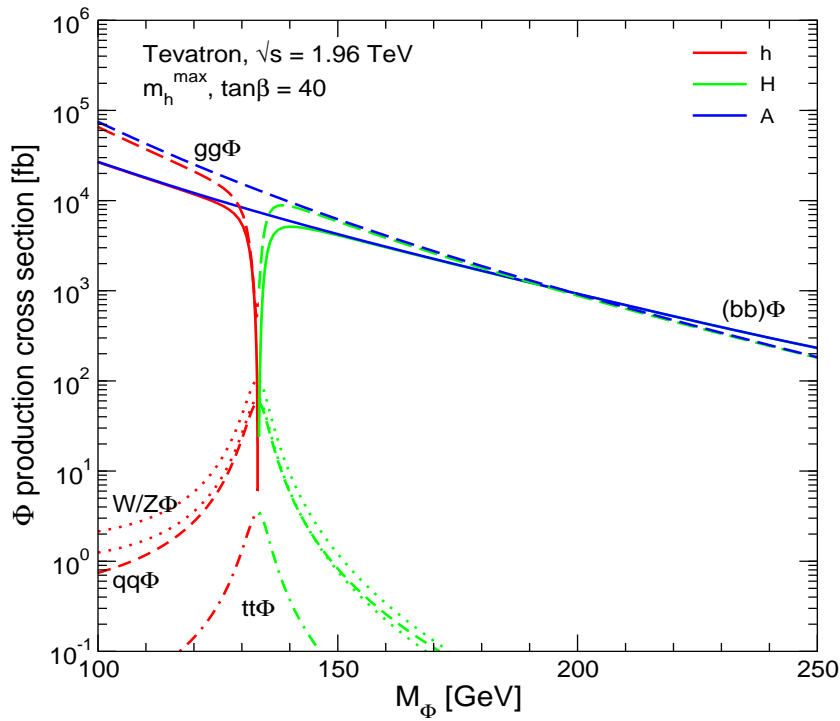

Fig. 17. MSSM Higgs boson production cross section as function of $m_{\phi}(\phi=h, H, A)$ for various production modes at $\tan \beta=40$ in the $m_{h}^{\max }$ scenario [21].

With the Tevatron running well, up to $\approx 6$ SM Higgs bosons events per day are produced per experiment, and the CDF and DØ Collaborations constantly improve their ability to find them. Combining CDF and $\mathrm{D} \varnothing$, about $4 \mathrm{fb}^{-1}$ could be sufficient to exclude the SM Higgs boson for $m_{H}=115 \mathrm{GeV}$ and $m_{H}=160 \mathrm{GeV}$ at $95 \%$ C.L. Assuming $7 \mathrm{fb}^{-1}$ of data analyzed by the end of the Tevatron running, all SM Higgs boson masses -except for the real mass value- could be excluded at 95\% C.L. up to $180 \mathrm{GeV}$.

\section{Higgs bosons in the MSSM}

In the minimal supersymmetric extension of the standard model, two Higgs doublets are necessary to cancel triangular anomalies and to provide masses to all particles. After electroweak symmetry breaking, the MSSM predicts 5 Higgs bosons. Three are neutral bosons: $h$, $H$ (scalar) and $A$ (pseudo-scalar), and two are charged bosons: $H^{+}$and $H^{-}$.

An important prediction of the MSSM is the theoretical upper limit $m_{h} \lesssim 135 \mathrm{GeV}$ on the mass of the lightest Higgs boson once the radiative loop corrections have been taken into account 71,72. All other relations between the Higgs masses and coupling are also significantly modified by the radiative corrections, which are dominated by the top- and stop-loop contributions [71,73. For large masses of the pseudo-scalar boson $A$, the light scalar Higgs becomes SM-like. The main difference between the MSSM Higgs bosons and the SM Higgs boson is the enhancement of the production cross section by a factor proportional to $\tan ^{2} \beta$, where $\tan \beta=v_{2} / v_{1}$ is the ratio of the vacuum expectation values associated with the two neutral components of the scalar Higgs fields. In contrast to the SM Higgs boson, the widths of the MSSM Higgs bosons do not exceed several tens of $\mathrm{GeV}$ in most of the scenarios. 


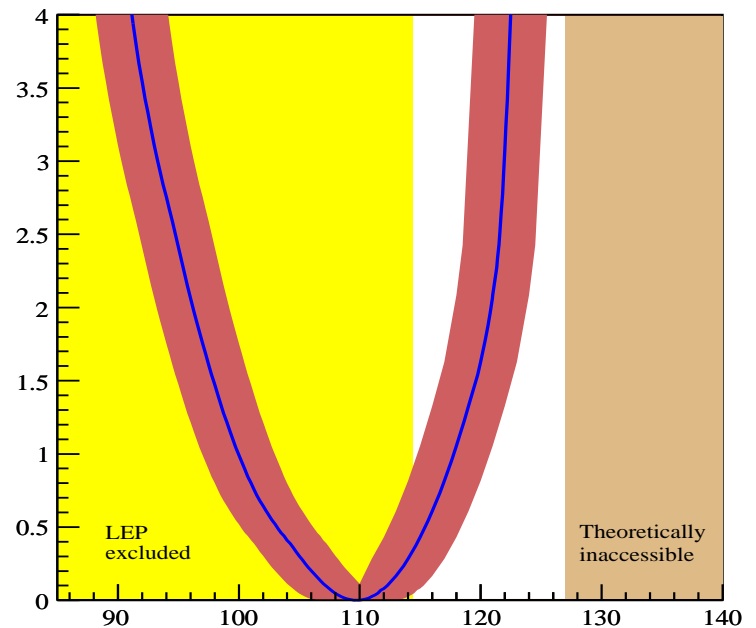

Fig. 18. Scan of the lightest Higgs boson mass versus $\Delta \chi^{2}$ derived from precision electroweak measurements in the context of a constrained MSSM model. The band around the $\Delta \chi^{2}$ curve represents the total theoretical uncertainty from unknown higher-order corrections and the dark shaded area on the right is theoretically inaccessible. The top mass $m_{t}=170.9 \pm 1.8$ was used for this analysis [8].

At tree level, only the mass $m_{A}$ and $\tan \beta$ are necessary to parameterize the Higgs sector in the MSSM. For $\tan \beta>1$, decays of $h$ and $A$ to $b \bar{b}$ and $\tau^{+} \tau^{-}$pairs are dominant with branching fraction of about $90 \%$ and $8 \%$, respectively. Although the branching fraction into $\tau$ 's is much smaller than the branching fraction into $b$ 's, the $\tau$ mode results in a much cleaner signature than the $b$ mode, as the latter suffers from a huge heavy-flavor multijet background which is poorly modeled by simulation.

Although most of the experimental searches at Tevatron assume $C P$ conservation $(C P C)$ in the MSSM sector, $C P$-violating $(C P V)$ effects can lead to sizable differences for the production and decay properties of the Higgs bosons compared to the $C P C$ scenario 74 . An observation of a new $C P V$ mechanism may yield insight into the observed abundance of matter over anti-matter in the universe.

\subsection{Search strategy}

At the Tevatron, $C P$ invariance is assumed for the searches. Both experiments have presented results on searches for neutral Higgs bosons in the two most promis ing final states:

$$
\begin{gathered}
p \bar{p} \rightarrow b \bar{b} \phi(h / H / A) \rightarrow b \bar{b} b \bar{b}, \\
p \bar{p} \rightarrow \phi(h / H / A) \rightarrow \tau^{+} \tau^{-} .
\end{gathered}
$$

The first process (11) corresponds to a neutral Higgs boson decaying into $b \bar{b}$ and produced in association with bottom quarks. The fourth $b$ is not required in the search, since a large fraction of the cross section produces a $b$-jet that does not pass the jet $E_{T}$ threshold. The second topology investigated at the Tevatron
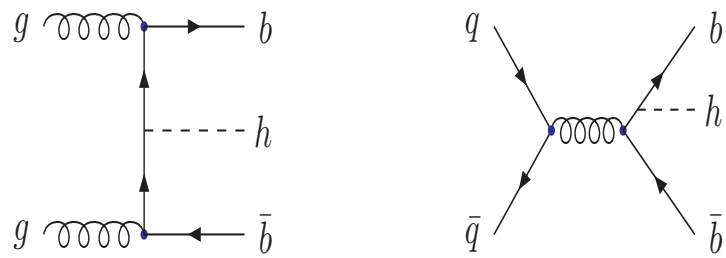

Fig. 19. Feynman diagrams contributing to the LO $g g \rightarrow$ $b \bar{b} h$ and $q \bar{q} \rightarrow b \bar{b} h$ MSSM Higgs boson production in association with bottom quarks.

is the gluon fusion process (2) where only the $\tau^{+} \tau^{-}$ mode is promising due to the overwhelming $b \bar{b}$ background.

The search strategy for charged MSSM Higgs bosons depends on their mass. For masses $m_{H^{ \pm}}<m_{t}-m_{b}$, the charged Higgs can be produced in the decay of the top quark $t \rightarrow b H^{+}$, which, in addition to the SM $t \rightarrow b W^{+}$decay, leads to the relevant production mode at the Tevatron:

$$
p \bar{p} \rightarrow t \bar{t} \rightarrow b H^{+} \bar{b} W^{-}
$$

The charged Higgs may decay to a variety of channels, with $H^{+} \rightarrow \tau^{+} \nu_{\tau}$ dominating for large values of $\tan \beta$. For values of the charged Higgs mass larger than the top mass, the dominant mode is charged Higgs production in association with a top and a bottom quark.

\subsection{Benchmark scenarios}

The choice of mechanism for mediating SUSY breaking and of the soft SUSY breaking terms governs the main phenomenological features of SUSY models. However, more than one hundred free parameters remain in the MSSM, rendering a complete scan virtually impossible. Several benchmark scenarios with simplifying assumptions have been therefore developed to interpret the experimental results.

The preliminary limits from CDF and D $\varnothing$ are available in the $\left(\tan \beta, m_{A}\right)$ plane and are usually summarized for two SUSY scenarios [75]. The $m_{h}^{\max }$ scenario is designed to maximize the allowed values of $m_{h}$ and therefore yields conservative exclusion limits. The nomixing scenario differs by the value (set to zero) of the parameter $X_{t}$ which controls the mixing in the stop sector, and hence leads to better limits.

Moreover, if one demands that the values of the bottom and $\tau$ Yukawa couplings remain in the perturbative regime up to energies of the order of the unification scale, the region $\tan \beta \gg 50$ in the MSSM is theoretically disfavoured [21]. In recent studies [76], negative values of the Higgsino mass parameter $(\mu)$ are also disfavoured.

The MSSM Higgs production cross section is shown in Fig. 17for $\tan \beta=40$ in the $m_{h}^{\max }$ scenario. At large $\tan \beta$, the pseudoscalar $A$ boson becomes degenerate with either the light $(h)$ or heavy $(H)$ scalar bosons 


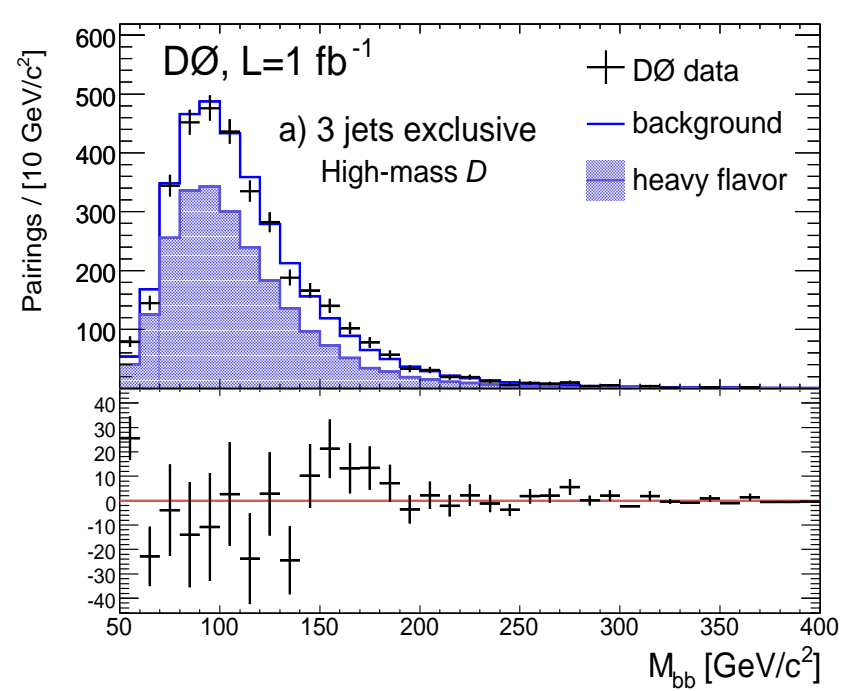

Fig. 20. Invariant mass for the high-mass likelihood region for the exclusive three-jet sample used to search for $p \bar{p} \rightarrow b \phi(h / H / A) \rightarrow b b \bar{b}+X$ in $1 \mathrm{fb}^{-1}$ of $\mathrm{D} \varnothing$ data 85 . The data are compared to the sum of total background processes (solid line) after all selections. The shaded region represents the heavy flavor component $(b \bar{b} b, b \bar{b} c, c \bar{c} b)$. The ratio between the data and the total background expectation is also shown.

and the bottom-Higgs coupling is enhanced. A production cross section in the $10 \mathrm{pb}$ range for the $(b b) \phi$ process is expected and could be observed at Tevatron.

Direct searches at LEP have placed lower mass limits on both the lightest scalar and pseudoscalar Higgs bosons at $m_{h, A}>93 \mathrm{GeV}$ at $95 \%$ C.L. 77 .

Similarly to the statistical analysis of precision electroweak measurements for the SM Higgs boson [19, a fit is performed in the context of a constrained MSSM model 78. The result is given in Fig. 18 as a one parameter scan in the lightest Higgs boson mass. The predicted value $m_{h}=110_{-10}^{+8}$ (exp.) \pm 3 (theo.) $\mathrm{GeV}$ agrees with the direct experimental lower limit from LEP of $114.4 \mathrm{GeV}[20$ and the upper theoretical bound.

\subsection{MSSM neutral Higgs bosons}

\subsection{1 $p \bar{p} \rightarrow b \phi(h / H / A) \rightarrow b b \bar{b}+X$}

At tree level (see Fig. 19), the cross section [21,79,80, for production of MSSM neutral Higgs bosons in association with bottom quarks is almost entirely dominated by the process $g g \rightarrow b \bar{b} h$, with only a small contribution from $q q \rightarrow b \bar{b} h$.

A search for $p \bar{p} \rightarrow b \phi(h / H / A) \rightarrow b b \bar{b}+X$ has been previously published by $\mathrm{D} \varnothing$ based on $260 \mathrm{pb}^{-1}$ [81. The results have been updated with $880 \mathrm{pb}^{-1}$ by the DØ Collaboration 82 and $980 \mathrm{pb}^{-1}$ by the CDF Collaboration 83]. For the winter 2008 conferences, CDF has presented a result with $1.9 \mathrm{fb}^{-1}$ of data 84 . The DØ Collaboration has just released for publication an improved analysis based on $1 \mathrm{fb}^{-1}$ of data [85].

In this analysis, CDF and $\mathrm{D} \varnothing$ search for an event signature of at least three b-jets with $p_{T}$ greater than
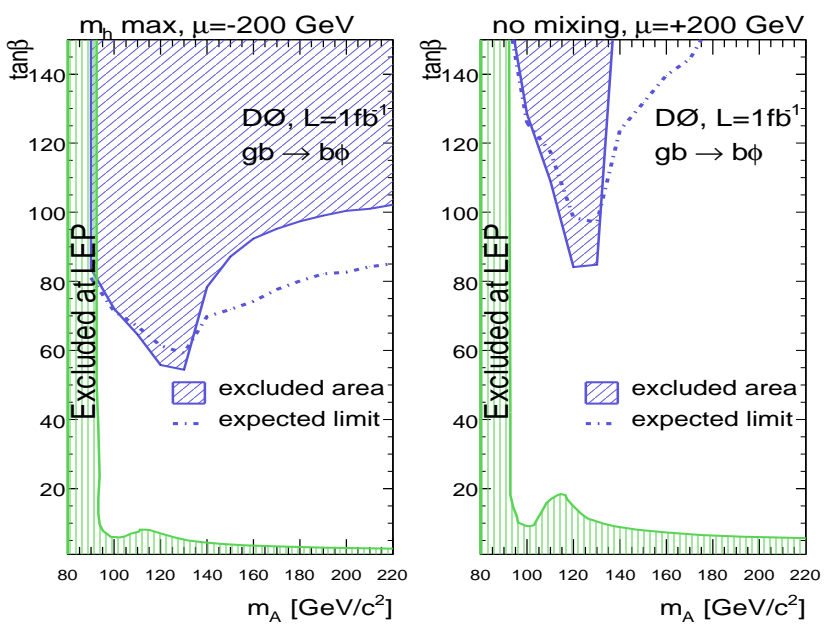

Fig. 21. D $\varnothing$ observed and expected $95 \%$ C.L. limits on $\tan \beta$ as a function of $m_{A}$ using $1 \mathrm{fb}^{-1}$ of data [85, assuming $\tan ^{2} \beta$ cross section enhancement. The effect of the Higgs width is included. The results are given for two scenarios: $m_{h}^{\max }$ with $\mu=-200 \mathrm{GeV}$, and no-mixing with $\mu=+200 \mathrm{GeV}$. The width of $\phi$ is larger than $70 \%$ above $\tan \beta=100$ in the $m_{h}^{\max }$ scenario with $\mu=-200 \mathrm{GeV}$. The exclusions from LEP are also displayed 77 .

$15 \mathrm{GeV}$. The events are triggered by using silicon tracking and jet requirements. The dijet mass spectrum of the two leading jets is used to separate the Higgs signal from background events.

A combination of data and simulation is used to model the background shape. The background in the three-tag sample is essentially all QCD heavy flavor multijet production. The sample consists of a mix of events: at least two real $b$-jets with the additional tagged jet being any of a mistagged light jet $(\approx 30 \%$ are $b \bar{b} j$ events where $j$ denotes a light parton: $u, d, s$ quark or gluon), a $c$-tag $(\approx 20 \%$ are $b \bar{b} c+b c \bar{c}$ events), or another $b$-jet $(\approx 50 \%$ are $b \bar{b} b$ events). In the three-jet sample, the double $b$-tagged events are found to be predominantly made of two real $b$-jets. This data sample of two $b$-tagged jets is exploited to predict the expected triple $b$-tagged background shape. Both CDF and D $\varnothing$ use only the shape, and not the normalization, of the final discriminating variable.

All Higgs signal events are simulated using the leading order PYTHIA 28] event generator. The cross section is corrected using next-to-leading order calculations from MCFM [35] for the Higgs $+b$ process. In addition, D $\varnothing$ corrects the signal acceptance to NLO. Weights obtained with MCFM are applied to the signal samples as function of $p_{T}$ and $\eta$ of the leading $b$-jet which is not from the decay of the Higgs boson.

A likelihood discriminant $(D)$ based on six kinematical variables built from the two leading jet-pair combinations is used by the $\mathrm{D} \varnothing$ search to separate the signal from the background. The invariant mass distribution for the exclusive three-tag sample is shown in Fig. 20 for the high-mass optimized likelihood cuts. To further increase the sensitivity, the analysis is also optimized in the four-jet and five-jet exclusive samples. 


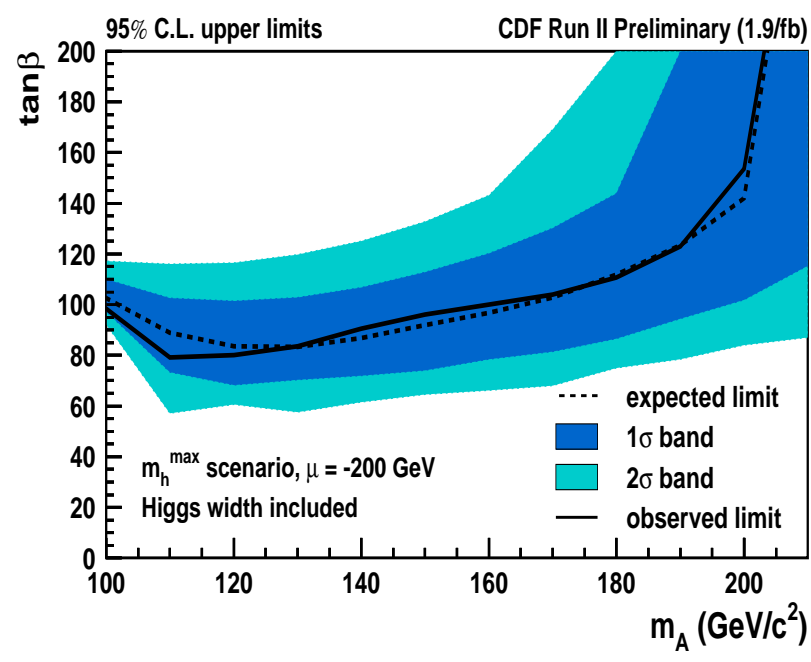

Fig. 22. The MSSM exclusion limit at $95 \%$ C.L. obtained by the CDF experiment with $1.9 \mathrm{fb}^{-1}$ of data on searches for neutral Higgs bosons produced in association with bottom quarks and decaying into $b \bar{b}$, projected onto the $\left(\tan \beta, m_{A}\right)$ plane assuming standard model cross section times branching fraction $(90 \%)$ with $\tan ^{2} \beta$ enhancement 84 . The error bands indicate the $\pm 1 \sigma$ and $\pm 2 \sigma$ range of the expected limit.

The CDF search uses a binned maximum-likelihood fit of two-dimensional templates in the mass of the two leading jets versus a variable sensitive to the flavor of the jet (based on the mass of the tracks forming the displaced vertex in the jets). The event selection efficiency varies as a function of the mass of the Higgs boson and is typically below $1 \%$.

The results have been interpreted in the context of MSSM models since no significant excess has been observed. Limits are placed on $\tan \beta$ versus the pseudoscalar mass $m_{A}$. The D $\varnothing$ and CDF exclusion contours, shown in Fig. 21 and Fig. 22, respectively, are based on the following approximate formula 86 :

$$
\begin{gathered}
\sigma(b \bar{b} A) \times \mathrm{BR}(\phi \rightarrow b \bar{b})= \\
\sigma(b \bar{b} A)_{\mathrm{SM}} \frac{\tan ^{2} \beta}{\left(1+\Delta_{b}\right)^{2}} \times \frac{9}{\left(1+\Delta_{b}\right)^{2}+9}
\end{gathered}
$$

where $\sigma(b \bar{b} A)_{\mathrm{SM}}$ denotes the value of the corresponding SM Higgs boson production cross section for a Higgs boson mass equal to $m_{A}$. The dependence of the exclusion bounds in the $\left(\tan \beta, m_{A}\right)$ plane on the parameters entering through the most relevant supersymmetric radiative corrections has been investigated. The loop effects, incorporated into the $\Delta_{b}$ parameter in the formula above, are discussed in Ref. 21. The bottom line is that their inclusion can enhance the cross section by $\approx \tan ^{2} \beta$ depending upon the MSSM scenario and significantly modify the bounds obtained. Negative values of $\mu$ will result in stronger limits on $\tan \beta$ since the $\Delta_{b}$ parameter is proportional to the product of $\tan \beta$ and $\mu$. In addition, $\mathrm{CDF}$ and $\mathrm{D} \varnothing$ take into account the effect of the Higgs boson width which is calculated with FEYNHIGGS 72 and included in the simulation as a function of the mass and $\tan \beta$ by convoluting a
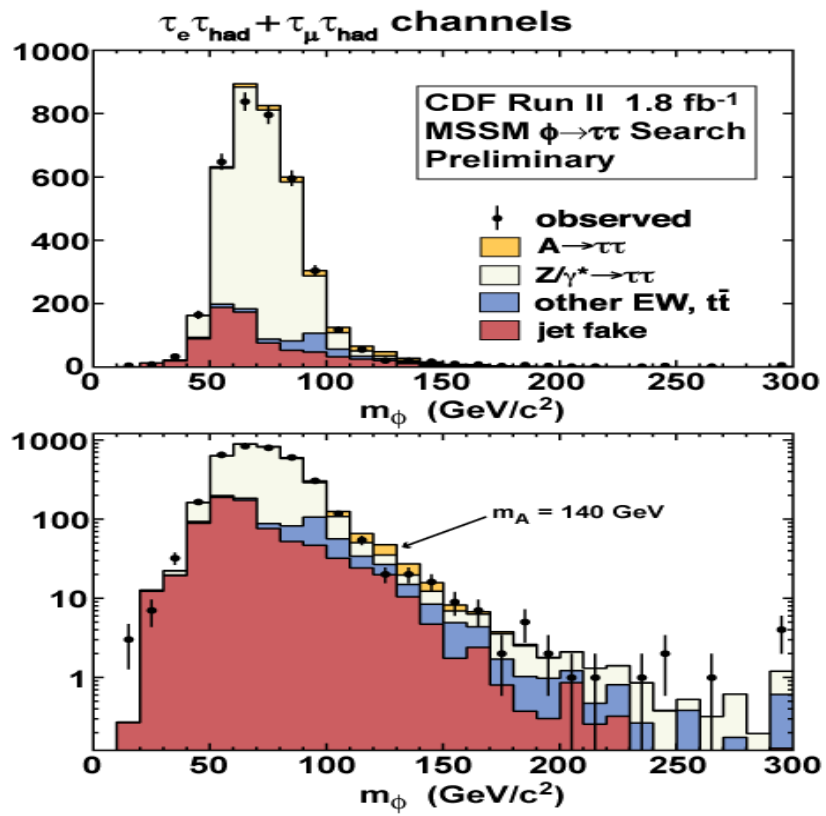

Fig. 23. Partially reconstructed ditau mass $\left(M_{v i s}=\right.$ $\sqrt{p_{T \ell}+p_{T_{\tau}}+E_{T}}$ ) from the CDF search for neutral MSSM Higgs boson production in the $\tau^{+} \tau^{-}$final state using $1.8 \mathrm{fb}^{-1}$ of data 90. Data (points with error bars) and expected backgrounds (filled histogram) are compared. The expected contribution from a signal at $m_{A}=140 \mathrm{GeV}$ is shown.

relativistic Breit-Wigner function with the NLO cross section. Currently the interpretation of these results within the MSSM framework is carried out using the program FEYNHIGGS. The cross section of FEYNHIGGS are based on a rescaling of the SM cross section by the corresponding MSSM factors of the Yukawa couplings. In future version of these analyses, comparisons with exact NLO calculations of the MSSM cross section for $g g \rightarrow$ Higgs should be considered using, for instance, the HIGLU program 87 .

In the $m_{h}^{\max }$ scenario with $\mu$ negative, the enhanced production through loop effects allows exclusion of $\tan \beta$ values greater than $60-100$ over the mass range 90 $210 \mathrm{GeV}$ for $m_{A}$.

The observed limits are within 2 standard deviations of the expectations over the mass region from 90 to $210 \mathrm{GeV}$, with the largest excess occurring around $160 \mathrm{GeV}$ and $180 \mathrm{GeV}$ in the CDF and D $\varnothing$ Collaboration searches, respectively.

\subsection{2 $p \bar{p} \rightarrow \phi(h / H / A) \rightarrow \tau^{+} \tau^{-}$}

The channels with $\tau^{+} \tau^{-}$final states have smaller signal branching fractions, but the searches do not suffer from the large multijet backgrounds that affect $\phi \rightarrow b \bar{b}$. In addition, compensations between large corrections in the Higgs production and decay reduce the impact of radiative corrections [21].

The published Run II CDF 88, (Dø 89]) results use 310 (348) $\mathrm{pb}^{-1}$ of data. The CDF Collaboration 


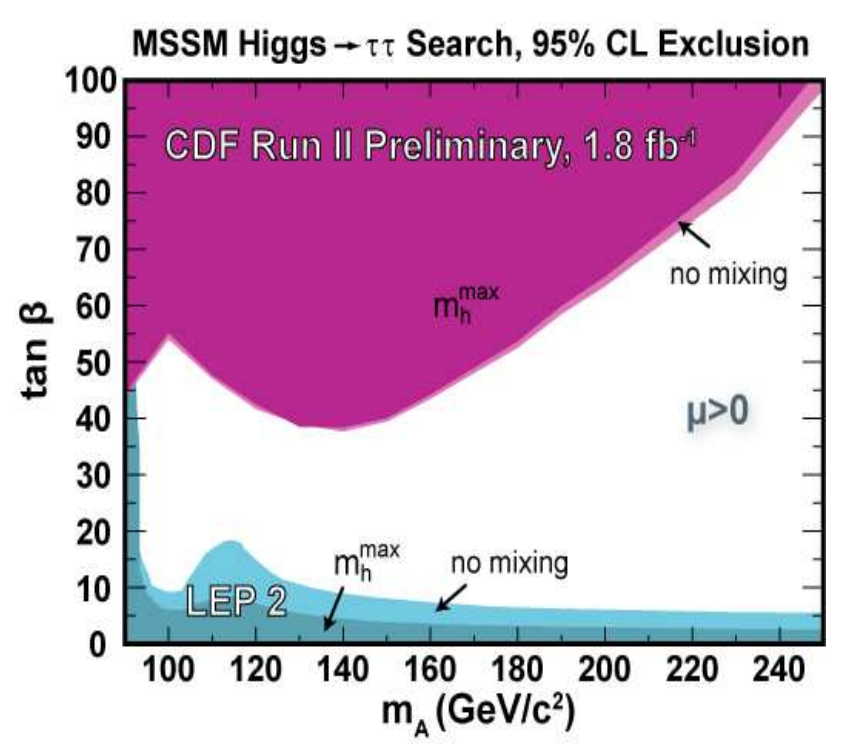

Fig. 24. MSSM neutral Higgs boson preliminary results in the $p \bar{p} \rightarrow \phi(h / H / A) \rightarrow \tau^{+} \tau^{-}$channels using $1.8 \mathrm{fb}^{-1}$ of CDF data 90 . The excluded regions in the $\left(\tan \beta, m_{A}\right)$ plane are shown for $\mu>0$. The Tevatron excluded domains in the $m_{h}^{\max }$ and no-mixing scenario are similar. Also shown are the regions excluded by LEP for these two scenarios 92 .

has recently released a new preliminary analysis with $1.8 \mathrm{fb}^{-1}$ [90. The $\mathrm{D} \varnothing$ Collaboration has just submitted for publication the result corresponding to $1 \mathrm{fb}^{-1}$ of data 91 .

Both CDF and DØ searches for inclusive production of neutral MSSM Higgs bosons are performed in three final states: $\tau_{e} \tau_{h}, \tau_{\mu} \tau_{h}$, and $\tau_{e} \tau_{\mu}$, where $\tau_{e}, \tau_{\mu}$, and $\tau_{h}$ are notations which stand for $\tau \rightarrow e \nu_{e} \nu_{\tau}, \tau \rightarrow$ $\mu \nu_{\mu} \nu_{\tau}$, and $\tau \rightarrow$ hadrons $\nu_{\tau}$, respectively. The decay products in $\tau_{h}$ appear as narrow jets with low track and $\pi^{0}$ multiplicity. The dominant and irreducible background in the final sample of selected events is from $Z / \gamma^{*}$ production with subsequent decays to $\tau$ pairs. Other sources of backgrounds are $W+$ jets, di-bosons, and fake jets. The DØ search uses neural networks to improve tau lepton identification. These neural networks make use of input variables that exploit the tau signature such as longitudinal and transverse shower shapes and isolation in the calorimeter and the tracker.

The CDF search probes for a possible Higgs signal by using a binned likelihood ratio of the partially reconstructed mass of the ditau system $\left(m_{v i s}\right)$, defined as the invariant mass of the visible tau decay products and the $\mathbb{E}_{T}$. Figure 23 shows $m_{v i s}$ in the $\tau^{+} \tau^{-}$ final state based on $1.8 \mathrm{fb}^{-1}$ of data collected by the CDF experiment. Since the data are consistent with a background only observation, limits are derived on the cross section for Higgs boson production times the branching fraction into tau leptons. The background contributions are allowed to float within limits set by Gaussian constraints corresponding to the systematic uncertainties. The corresponding excluded regions in the $\left(\tan \beta, m_{A}\right)$ plane are shown in Fig. 24] for the case

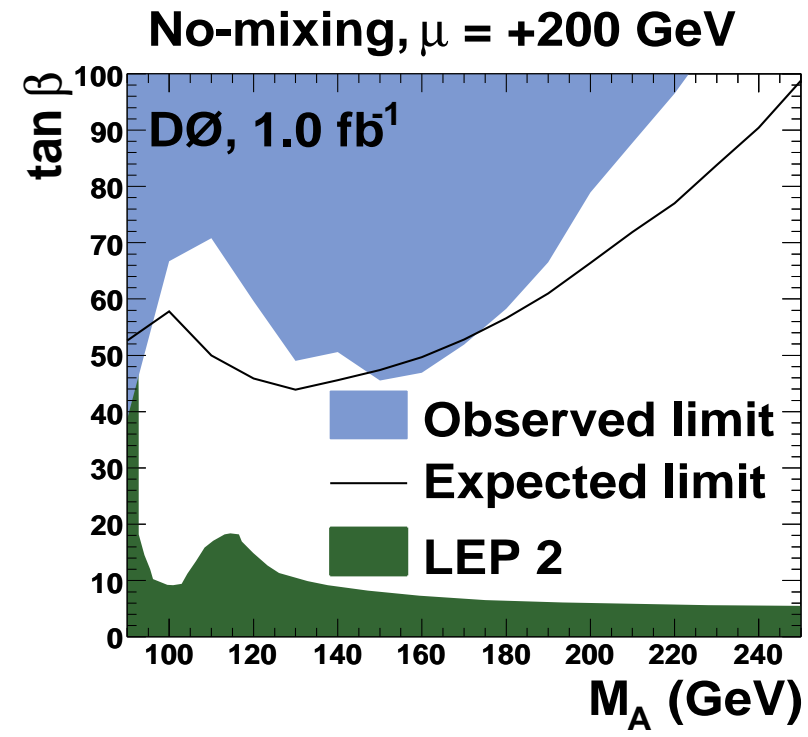

Fig. 25. D $\varnothing$ MSSM exclusion limits at 95\% C.L. obtained with $1 \mathrm{fb}^{-1}$ of data on searches for neutral MSSM Higgs bosons decaying to tau pairs 91 . The $\left(\tan \beta, m_{A}\right)$ plane is shown for the no-mixing scenario. The excluded region at LEP is also represented [77].

$\mu>0$. The dependence of the $\tan \beta$ bounds on the sign of $\mu$ can be as large as $\Delta \tan \beta \sim 30$ for the $m_{h}^{\max }$ scenario, while in the case of the no-mixing scenario its effect is smaller, of the order of $\Delta \tan \beta \sim 10$ [21]. The cross sections are taken from [86] and are obtained from SM calculations and scaling factors $\sigma_{\mathrm{MSSM}} / \sigma_{\mathrm{SM}}$ accounting for the modified Higgs couplings.

The $\mathrm{D} \emptyset$ search based on $1 \mathrm{fb}^{-1}$ of data exploits the full $m_{v i s}$ spectrum for the likelihood ratio limit calculation. Limits on $\tan \beta$ as a function of $m_{A}$ are derived for the $m_{h}^{\max }$ and no-mixing scenarios, where only positive values of $\mu$ are considered. The result corresponding to the no-mixing scenario is represented in Fig. 25. A sensitivity of $\tan \beta \approx 50$ for $m_{A}$ below $180 \mathrm{GeV}$ is obtained. The difference between the observed and expected limits are within two standard deviations or slightly above for $m_{A}>250 \mathrm{GeV}$. It is mainly caused by a data excess in the $\mu \tau_{h}$ channel for $m_{v i s}>160 \mathrm{GeV}$. The distributions of $m_{v i s}$ in the three final states are shown in Fig. 26 .

The combination of the LEP and Tevatron searches for neutral Higgs bosons is expected to probe vast regions of the $\left(\tan \beta, m_{A}\right)$ plane by the end of the Run II. In the no-mixing scenario shown in Fig. 24] and Fig. 25, the lower limits on $\tan \beta$ obtained at LEP 92 will slightly increase because of the assumed top mass $m_{t}=174.3 \mathrm{GeV}$, which is higher than the currently measured value $m_{t}=172.6 \pm 1.4 \mathrm{GeV}$ [16]. The Tevatron results are not sensitive to the precise value of the top mass. The upper limits will also extend with the growing data samples, together with the improvement of the CDF and D $\varnothing$ searches. The projected sensitivity on the excluded domain would potentially allow exclu- 


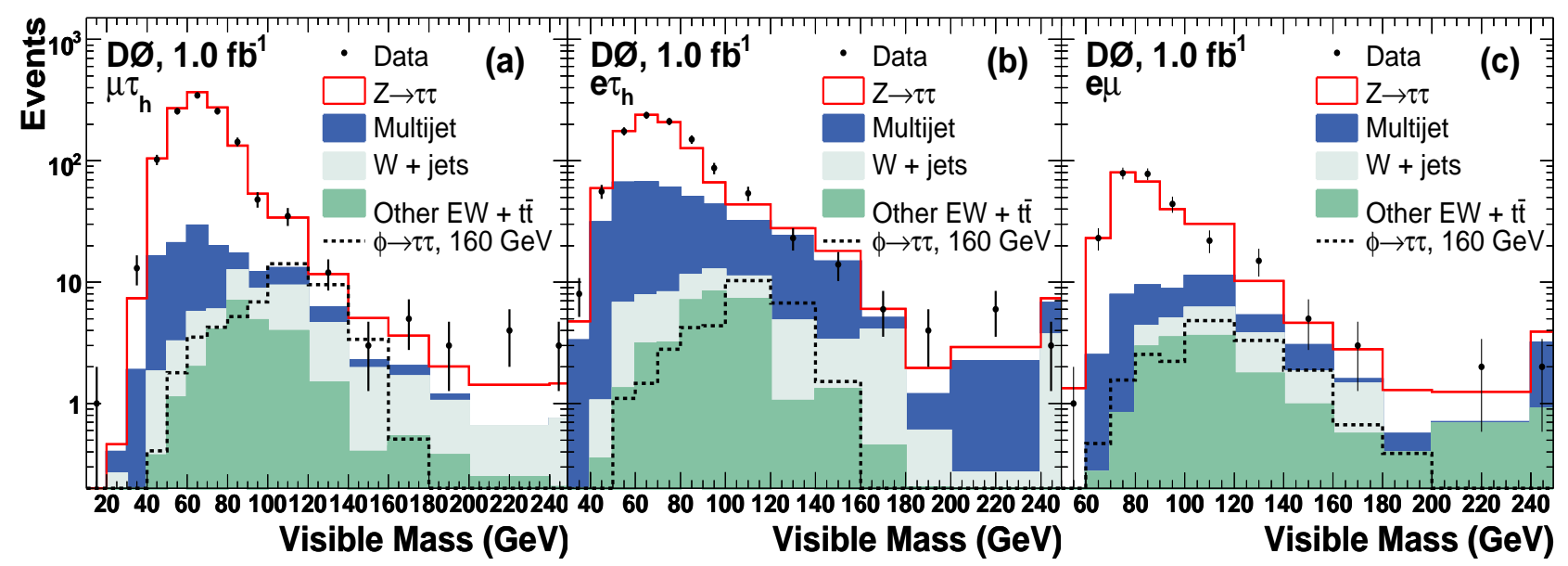

Fig. 26. D $\varnothing$ visible mass $\left(m_{v i s}\right)$ distributions used to search for neutral MSSM Higgs bosons decaying to tau pairs in the (a) $\mu \tau_{h}$, (b) $e \tau_{h}$, and (c) $e \mu$ channels with $1 \mathrm{fb}^{-1}$ of data [91. The signal $\phi \rightarrow \tau^{+} \tau^{-}$corresponds to $m_{\phi}=160 \mathrm{GeV}$ and is normalized to a cross section of $3 \mathrm{pb}$. The highest bin includes the overflow.

sion of $\tan \beta>20$ for values of $m_{A}$ up to few hundred $\mathrm{GeV}$.

\subsection{Charged Higgs bosons}

\subsection{1 $t \rightarrow b H^{+}$}

At the Tevatron, direct production of single charged Higgs bosons is expected to have negligible rate, and the direct production of $\mathrm{H}^{+} \mathrm{H}^{-}$via the weak interaction is expected to have a relatively small cross section, on the order of $0.1 \mathrm{pb}$ [73. However, more significant production could be obtained in the decay of the top quark $t \rightarrow b H^{+}$, which would compete with the SM process $t \rightarrow b W^{+}$. The only recent search for $t \rightarrow b H^{+}$ at Run II is from the CDF Collaboration, which has published a result based on $193 \mathrm{pb}^{-1}$ of data 93 . The CDF search excludes the top quark branching fraction to a charged Higgs boson and $b$-quark $B R\left(t \rightarrow H^{+} b\right)>$ 0.4 at $95 \%$ C.L. in the region $80 \mathrm{GeV}<m_{H^{ \pm}}<$ $160 \mathrm{GeV}$, assuming $B R\left(H^{+} \rightarrow \tau^{+} \nu_{\tau}\right)=1$. Another search, interpreted in the context of the MSSM model, was for anomalous production of high transverse momentum tau leptons in the decay products of pairproduced top quarks using $335 \mathrm{pb}^{-1}$ of data taken with the CDF detector in Run II 94. An upper limit on $B R\left(t \rightarrow H^{+} b\right)>0.34$ at $95 \%$ C.L. is set for a charged Higgs mass of $120 \mathrm{GeV}$.

\subsubsection{Doubly charged Higgs bosons}

Doubly charged Higgs bosons $H^{ \pm \pm}$are predicted in many scenarios, such as left-right symmetric models [95, Higgs triplet models and little Higgs models 96 . 97.

Limits on doubly charged Higgs bosons have been published in the $e e, e \mu$, and $\mu \mu$ channels by the CDF 98 (DØ [99]) experiment based on $240 \mathrm{pb}^{-1}\left(113 \mathrm{pb}^{-1}\right)$

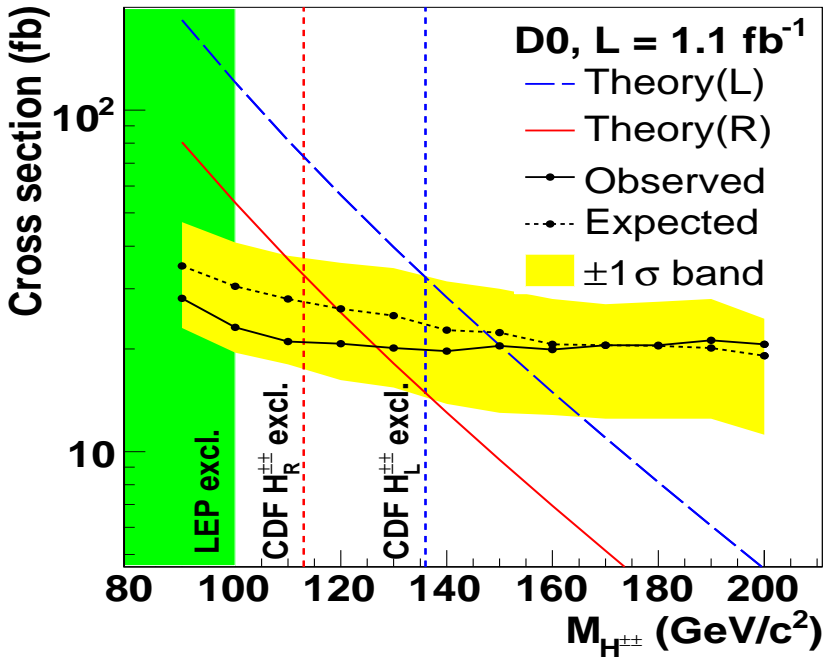

Fig. 27. Cross section limit as a function of the doubly charged Higgs mass $M_{H^{ \pm \pm}}$at the $95 \%$ C.L. in the $\mu^{+} \mu^{+} \mu^{-} \mu^{-}$final state using $1 \mathrm{fb}^{-1}$ of data collected by the $\mathrm{D} \varnothing$ detector at Run II [101]. The mass regions excluded by CDF 98 and LEP 102 are also shown. The $\pm 1 \sigma$ uncertainty on the expected limit is represented by the band.

of data collected at Tevatron Run II. The search for $p \bar{p} \rightarrow H^{++} H^{--} \rightarrow \ell^{+} \tau^{+} \ell^{-} \tau^{-}$final states has also been performed by CDF [100] with $350 \mathrm{pb}^{-1}$. The DØ Collaboration has recently submitted for publication a search for $H^{ \pm \pm}$in the $\mu^{+} \mu^{+} \mu^{-} \mu^{-}$final state using $1 \mathrm{fb}^{-1}$ of data 101, and sets lower bounds for right$\left(H_{R}^{ \pm \pm}\right)$and left-handed $\left(H_{L}^{ \pm \pm}\right)$bosons at $126 \mathrm{GeV}$ and $150 \mathrm{GeV}$, respectively, at $95 \%$ C.L. This result is shown in Fig. 27, In addition, CDF has published [103, with $292 \mathrm{pb}^{-1}$ of data the case where the doubly charged Higgs boson lifetime is long $(>3 \mathrm{~m})$, such that it decays outside the detector. The lower mass bound on long-lived doubly charged $H_{L}^{ \pm \pm}$and $H_{R}^{ \pm \pm}$bosons are 


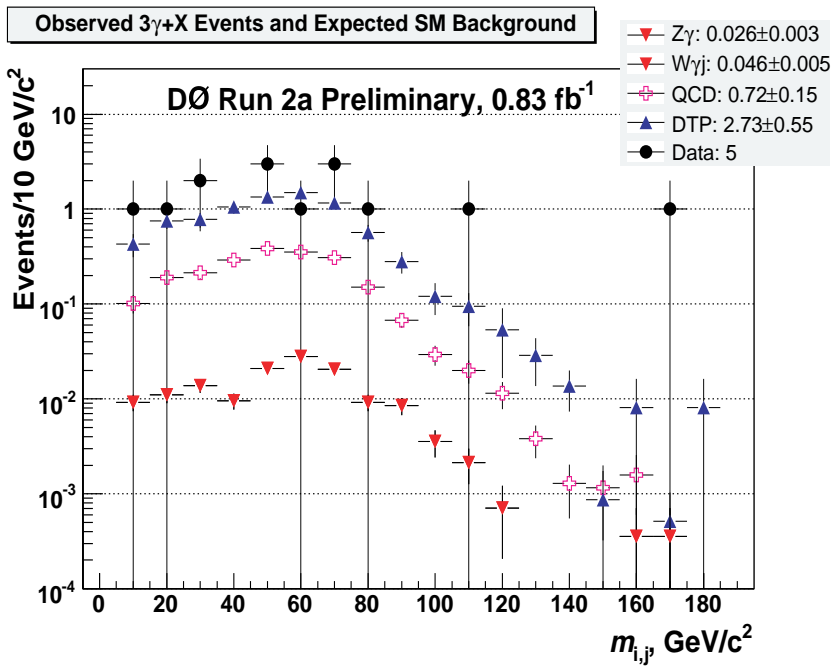

Fig. 28. Distribution of two-body invariant mass for $\gamma \gamma \gamma+$ $X$ events observed in $0.83 \mathrm{fb}^{-1}$ of $\mathrm{D} \varnothing$ data along with the expected SM background [104]. The $2.73 \pm 0.55$ events are estimated from direct triphon production (DTP). This analysis is used to search for fermiophobic Higgs via the process $p \bar{p} \rightarrow h_{f} H^{ \pm} \rightarrow h_{f} h_{f} \rightarrow \gamma \gamma \gamma(\gamma)+X$.

$133 \mathrm{GeV}$ and $109 \mathrm{GeV}$, respectively. When the two states are degenerate in mass, the limit is increased to $m_{H^{ \pm \pm}}<146 \mathrm{GeV}$ at $95 \%$ C.L.

\section{Extended Higgs models}

In a more general framework, one may expect deviations from the SM predictions to result in significant changes to the Higgs boson discovery signatures. One such example is the so-called "fermiophobic" Higgs boson [51,52,53, which has suppressed couplings to all fermions.

Experimental searches for fermiophobic Higgs at LEP and Tevatron have yielded negative results so far. In fermiophobic models, the decay $H^{ \pm} \rightarrow h_{f} W^{*}$ can have a larger branching fraction than the conventional decays $H^{ \pm} \rightarrow t b, \tau \nu$. This would lead to double $h_{f}$ production. Searches have been conducted via the process $p \bar{p} \rightarrow h_{f} H^{ \pm} \rightarrow h_{f} h_{f} W^{ \pm} \rightarrow \gamma \gamma \gamma(\gamma)+X$ by the D $\varnothing$ experiment using $0.83 \mathrm{fb}^{-1}$ of data 104 . Figure 28 shows the distribution of the diphoton invariant mass in data and from the expected backgrounds, where each event contributes three histogram entries since they are three possible photon-photon combinations. This analysis select 5 events in the data. The $2.73 \pm$ 0.55 background events from direct triphon production (DTP), i.e., direct diphoton production (DDP) along with the FSR/ISR photon, are estimated by scaling the corrected number of diphoton events observed in data with the rate at which one would expect to observe a third photon in DDP processes from PYTHIA. The background from events in which jets or electrons were misidentified as photons is estimated in data and represents $0.8 \pm 0.15$ event. In absence of excess, a limit

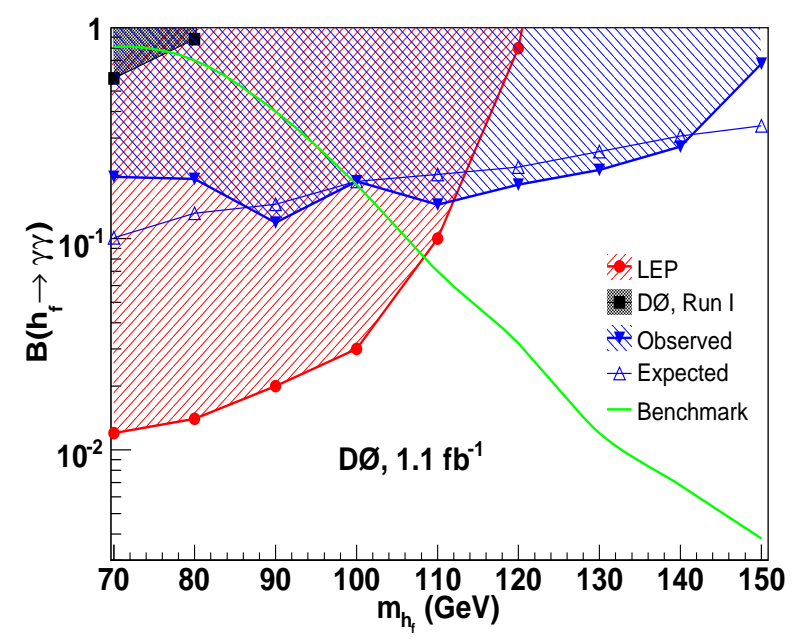

Fig. 29. Excluded branching fractions $B\left(h_{f} \rightarrow \gamma \gamma\right)$ as function of the fermiophobic Higgs boson mass $m_{h_{f}}$ for the D $\varnothing$ search with $1.1 \mathrm{fb}^{-1}$ of data 62 . The signal is the sum of $p \bar{p} \rightarrow V V \rightarrow h_{f}$ and $p \bar{p} \rightarrow h_{f} V$ processes. The theoretical curve uses the benchmark scenario assuming that the coupling $h_{f} V V(V=W, Z)$ has the same strength as in the SM and that all fermion branching fractions are exactly zero.

is set at $m_{h_{f}}>80 \mathrm{GeV}$ for $m_{H^{ \pm}}<100 \mathrm{GeV}$ and $\tan \beta=30$ at $95 \%$ C.L.

Another D $\varnothing$ search for fermiophobic Higgs bosons has been recently submitted for publication using 1.1 $\mathrm{fb}^{-1}$ of data 62 . This analysis searches for inclusive production of diphoton final states via Higgsstrahlung $p \bar{p} \rightarrow h_{f} V \rightarrow \gamma \gamma+X$ and vector boson fusion $p \bar{p} \rightarrow V V$ $\rightarrow \gamma \gamma+X$ processes $(V=W, Z)$. The benchmark model used to set mass limits assumes that the coupling $h_{f} V V$ has the same strength as in the SM and that all fermion branching fractions are exactly zero. The study shown in Fig. 29 excludes fermiophobic Higgs bosons of mass up to $100 \mathrm{GeV}$ at the $95 \%$ C.L. and represents the most stringent limits to date at a hadron collider.

Technicolor models 105 provide an alternative dynamical explanation of electroweak symmetry breaking through a new strong gauge interaction acting on new fermions, called "techni-fermions". The D $\varnothing$ Collaboration published a search corresponding to 390 $\mathrm{pb}^{-1}$ of data 106 in the final state containing one electron and two jets coming from the decay of vector techni-mesons $\left(p \bar{p} \rightarrow \rho_{T} / \omega_{T}\right)$ to a $W$ boson and a techni-pion $\pi_{T}$, followed by the decays $W \rightarrow e \nu$ and $\pi_{T} \rightarrow b \bar{b}, b \bar{c}$, or $c \bar{c}$. As no significant excess in the data was observed, limits have been set. For instance, a mass of $m_{\rho_{T}} \approx 210 \mathrm{GeV}$ is excluded for the corresponding $m_{\pi_{T}} \approx 120 \mathrm{GeV}$ at $95 \%$ C.L. Similarly, CDF presented a search for technicolor particles decaying into $b \bar{b}, b \bar{c}$ or $b u$ and produced in association with $W$ bosons using $1.9 \mathrm{fb}^{-1}$ of data 107. Events matching the $W+2$ jets signature are selected by requiring the electron or muon to be isolated with $E_{T}$ or $p_{T}>$ $20 \mathrm{GeV}, \not_{T}>20 \mathrm{GeV}$, and at least one $b$-tagged jet. The number of tagged events and the invariant mass 
distributions of $W+2$ jets and dijet events are consistent with the SM expectations. For $m_{\rho_{T}} \approx 250 \mathrm{GeV}$, the excluded mass range is $135 \mathrm{GeV}<m_{\pi_{T}}<145 \mathrm{GeV}$ at $95 \%$ C.L.

\section{Beyond the standard model}

Beyond the elucidation of the mechanism of electroweak symmetry breaking, there are many compelling and well-motivated models that can be tested at the Tevatron. But what are the CDF and D $\varnothing$ Collaborations looking for at Tevatron? By far, the most widely studied theory beyond the SM involves new particles predicted by low energy supersymmetry. Searches are therefore often divided into SUSY and non-SUSY categories. This succinct summary covers the following topics:

- Extension beyond the Poincaré group, i.e., supersymmetry, such as searches for electroweak gauginos with leptonic decay, and squark and gluino production resulting in multijet $+\mathbb{E}_{T}$ topologies.

- Existence of a new symmetry leading to massive particles with a lifetime comparable to the typical transit time through the detector.

- Particle substructure or compositeness, such that history repeats itself, leading to e.g., leptoquark particles or excited fermion states.

- An enlarged gauge group resulting in exotic $Z^{\prime}$ or $W^{\prime}$ bosons.

- An increase in the number of spacial dimensions, i.e., extra-dimension models with real Kaluza-Klein gravitons produced in association with a jet, or virtual Kaluza-Klein gravitons exchanged in the production of fermion or vector-boson pairs.

- A search for an excess in data without a specific model in mind. These are grouped into so-called signature-based searches.

\section{Charginos and neutralinos}

Charginos and neutralinos are respectively the charged and neutral partners of gauge and Higgs bosons. The primary search modes are pair production of charginos $\left(\chi_{1}^{+} \chi_{1}^{-}\right)$or associated chargino-neutralino $\left(\chi_{1}^{ \pm} \chi_{2}^{0}\right)$ production, where $\chi_{1}^{ \pm}$is the lightest chargino and $\chi_{2}^{0}$ is the second lightest neutralino. A search for SUSY can therefore be performed via the associated production of charginos and neutralinos, where the $\chi_{1}^{ \pm}$and $\chi_{2}^{0}$ are assumed to decay either via exchange of vector bosons or via sleptons into SM fermions while the lightest neutralino remains undetected.

The CDF and DØ Collaborations have searched in the trilepton final state. Published results are based on $320 \mathrm{pb}^{-1}$ for $\mathrm{D} \varnothing 108$ and $1.1 \mathrm{fb}^{-1}$ for CDF 109, 110 , 111. The eel channel was updated to $1.7 \mathrm{fb}^{-1}$ by $\mathrm{D} \varnothing$ and, in combination with results in four other trilepton search channels based on approximately $1 \mathrm{fb}^{-1}$ of data, new limits on the associated production of charginos and neutralinos have been set by the $\mathrm{D} \varnothing$ Collaboration

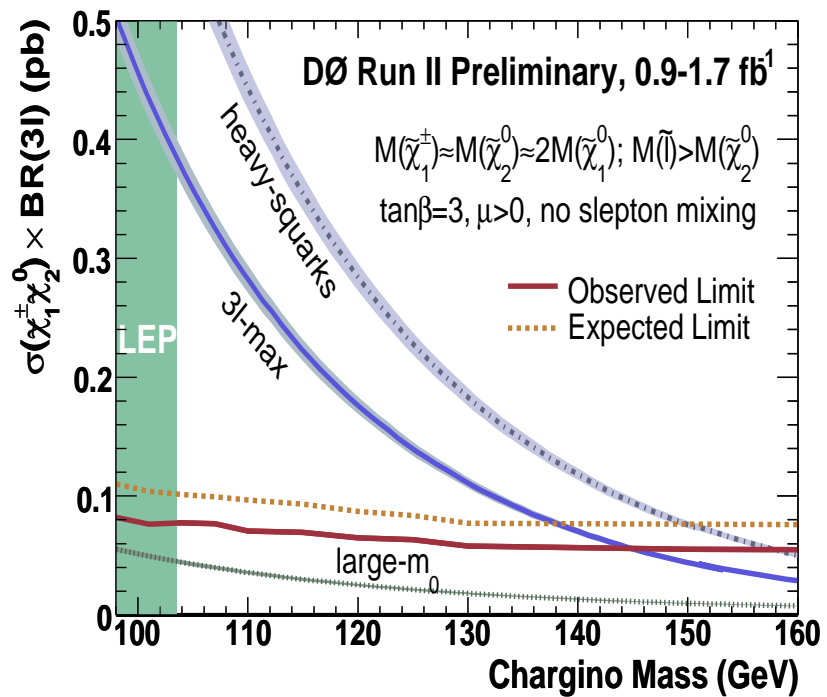

Fig. 30. DØ 95\% C.L. limits on the total cross section for associated chargino and neutralino production with leptonic final states as a function of the chargino $\left(\chi_{1}^{ \pm}\right)$mass, in comparison with the expectation for several SUSY scenarios using $0.9-1.7 \mathrm{fb}^{-1}$ of data [112. The line corresponds to observed minimal SUGRA limit. PDF and renormalization/factorization scale uncertainties are shown as shaded bands. The lower mass limit at $103.5 \mathrm{GeV}$ is from LEP searches 114 .

112. The CDF results were updated with $2 \mathrm{fb}^{-1}$ of data $[113$.

The trilepton final state has long been suggested to be one of the most promising channel for discovery of SUSY at a hadron collider. However, these searches suffer from a cross section below $0.5 \mathrm{pb}$ with leptons that are difficult to reconstruct due to their low transverse momenta, rendering the analyses challenging. Furthermore, many channels need to be combined to achieve sensitivity. The selection consists of two well identified and isolated electrons $(e)$ or muons $(\mu)$ with a $p_{T}$ cut above $\approx 10 \mathrm{GeV}$. An additional isolated track provides sensitivity to the third lepton $(l)$ and, by not requiring explicit lepton identification, efficiency is maximized. The presence of neutrinos and neutralinos in the final state results in some missing transverse energy. Finally, since very few SM processes are capable of generating a pair of isolated like-charge leptons, the same analysis is performed with this looser criterion.

The results are interpreted in minimal SUGRA inspired scenarios (mSUGRA) where gravity mediates SUSY breaking from the grand unification theory (GUT) scale to the electroweak scale. With $R$-parity conservation (see section 11), mSUGRA can be completely characterized by five parameters: a common scalar mass $\left(m_{0}\right)$, a common gaugino mass $\left(m_{1 / 2}\right)$, a common trilinear coupling value $\left(A_{0}\right)$, the ratio of the vacuum expectation values of the two Higgs doublets $(\tan \beta)$, and the sign of the Higgsino mass parameter $(\mu)$. Direct searches at LEP set a lower limit on the mass of the chargino $\chi_{1}^{ \pm}$at $103.5 \mathrm{GeV}$ for sneutrino masses larger than $200 \mathrm{GeV}$ [114. 


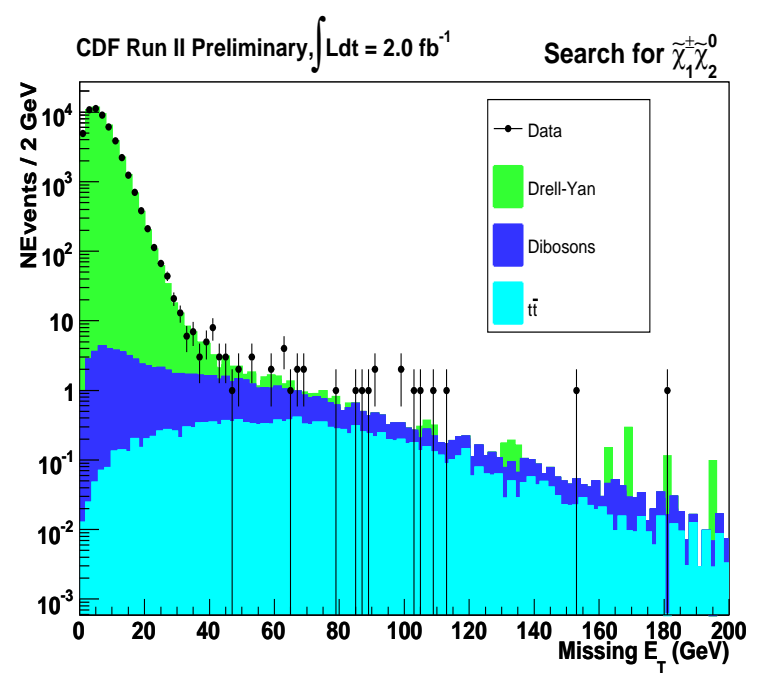

Fig. 31. $\mathbb{E}_{T}$ distribution for di-lepton events with invariant mass $76 \mathrm{GeV}<m_{\ell \ell}<106 \mathrm{GeV}$. This control region is used to test SM predictions of the CDF search for charginos and neutralinos using $2 \mathrm{fb}^{-1}$ of data 113 .

As a guideline, $\mathrm{D} \varnothing$ results are interpreted in this model with chargino $\chi_{1}^{ \pm}$and neutralino $\left(\chi_{2}^{0}, \chi_{1}^{0}\right)$ masses following the relation $m_{\chi_{1}^{ \pm}} \simeq m_{\chi_{2}^{0}} \simeq 2 m_{\chi_{1}^{0}}$. The leptonic branching fraction of chargino and neutralino depends on the relative contribution from the sleptonand $\mathrm{W} / \mathrm{Z}$-exchange graphs, which varies as a function of the slepton masses. Three mSUGRA inspired scenarios were used for the interpretation as shown in Fig. 30. Two of them are with enhanced leptonic branching fractions ("heavy squarks" and "3l-max" scenarios). For the $3 l$-max scenario, the slepton mass is just above the neutralino mass $\left(m_{\chi_{2}^{0}}\right)$, leading to maximum branching fraction into leptons. The heavy squark scenario is characterized by maximal production cross section. Finally, the large universal scalar mass parameter $\left(m_{0}\right)$ scenario is not yet sensitive because the $W / Z$ exchange dominates. The new $\mathrm{D} \varnothing$ result 112 in the eel channel using $1.7 \mathrm{fb}^{-1}$ of data observes no events after final selection, with $1.0 \pm 0.3$ events expected from the SM background and between 0.5-0.2 events for the signal. In the dataset corresponding to $1 \mathrm{fb}^{-1}$, no candidates have been found in the $e \mu \ell$ channel with an expected background of $0.9_{-0.1}^{+0.4}$ events, while two candidates are found in the $\mu \mu \ell$ channel consistent with the background expectation of $0.3_{-0.1}^{+0.7}$ events. In the eel channel, no candidates have been found, with an expected background of $0.8 \pm 0.7$ events. The observation of one event in the data is consistent with the $1.1 \pm 0.4$ events expected from the background in the $\mu^{ \pm} \mu^{ \pm}$channel. Since no evidence for SUSY is reported, all results are combined to extract limits on the total cross section, taking into account systematic and statistical uncertainties including their correlations. The $\mathrm{D} \varnothing$ combination excludes chargino masses below $145 \mathrm{GeV}$ at $95 \%$ C.L. for the $3 l$-max scenario.

Similar analyses have been performed by CDF but interpreted with slightly different scenarios and with

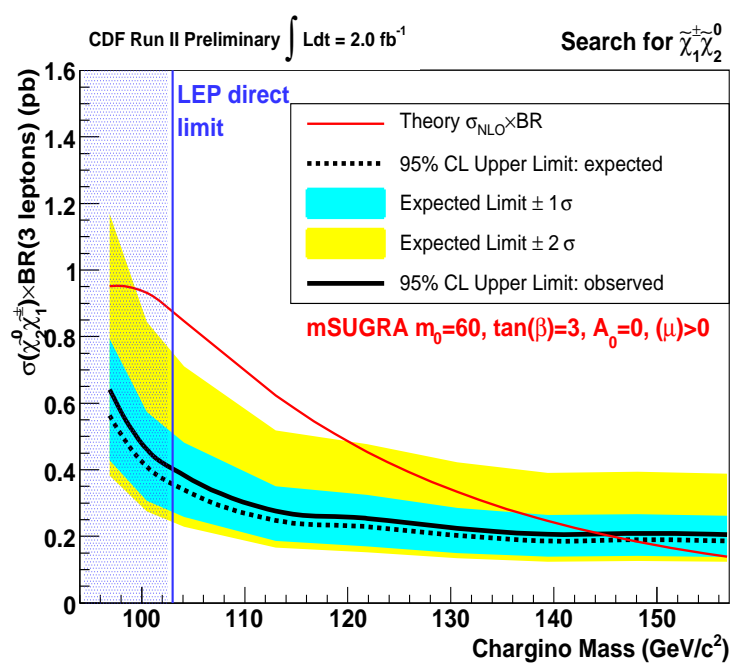

Fig. 32. CDF 95\% C.L. limits on the total cross section for associated chargino and neutralino production with leptonic final states using $2 \mathrm{fb}^{-1}$ of data 113 . The expected limit corresponds to the dashed line, with $\pm 1 \sigma$ and $\pm 2 \sigma$ uncertainty bands shown. The next-to-leading order (NLO) production cross section corresponds to an mSUGRA model with the universal scalar mass parameter fixed to $m_{0}=60 \mathrm{GeV}$.

a total integrated luminosity corresponding to $2 \mathrm{fb}^{-1}$ of data [113]. After selecting dilepton events, a control region with $76 \mathrm{GeV}<m_{\ell \ell}<106 \mathrm{GeV}$ is used to establish a good understanding of the data and to test the SM predictions. The $\mathbb{E}_{T}$ distribution for this control region is displayed in Fig. 31, The low and high invariant mass regions are also explored. The search is splitted into five exclusive channels and optimized for a benchmark signal point in the minimal SUGRA scenario, corresponding to $m_{0}=60 \mathrm{GeV}, m_{1 / 2}=190 \mathrm{GeV}$, $\tan \beta=3, A_{0}=0$, and $\mu>0$. In this case, the masses $m_{\chi_{1}^{ \pm}}=119.6 \mathrm{GeV}, m_{\chi_{1}^{0}}=122 \mathrm{GeV}, m_{\chi_{2}^{0}}=67 \mathrm{GeV}$ have been computed with ISAJET [115] and the corresponding $\chi_{1}^{ \pm} \chi_{2}^{0}$ production cross section $0.327 \mathrm{pb}$ with PROSPINO- 2 [116]. A total of $0.9 \pm 0.1$ background events in the trilepton channels are expected for $4.5 \pm$ 0.4 events from the signal, and $5.5 \pm 1.1$ background events for the dilepton+track channels for $6.9 \pm 0.6$ signal events. CDF observes 1 event in the trilepton channel and 6 events in the dilepton+track channels. No excess is therefore observed and the resulting cross section limit shown in Fig. 32 is given as a function of the chargino mass for the benchmark mSUGRA scenario defined above but varying $m_{1 / 2}$. This scenario enhances the branching fraction of chargino and neutralino into leptons, and excludes chargino masses below $140 \mathrm{GeV}$ for a sensitivity (expected limit) of $142 \mathrm{GeV}$ at $95 \%$ C.L. Other models are being investigated for upcoming analyses with increased luminosity.

The results between the two experiments cannot be directly compared since the fixed low $m_{0}$ value leads to a two-body decay for the CDF analysis, while for the $\mathrm{D} \varnothing$ analysis a sliding window of $m_{0}$ is used to 


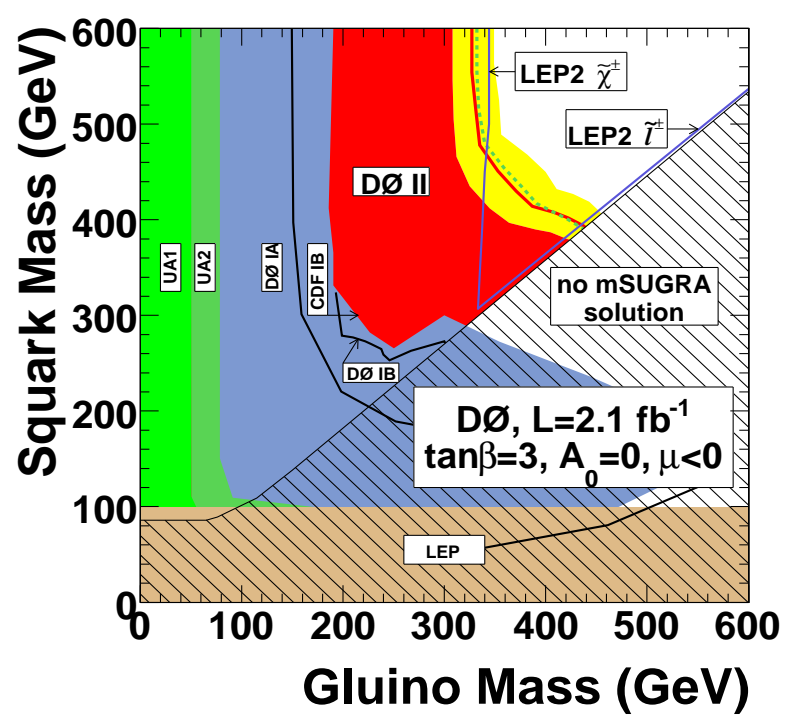

Fig. 33. DØ Run II exclusion plane for squark and gluino masses at $95 \%$ C.L. using $2.1 \mathrm{fb}^{-1}$ of Run II data [17], in the mSUGRA framework. The region excluded by previous results and this analysis is shown as the "DØ II" shaded area. The thick (dotted) line is the limit of the observed (expected) excluded region for the nominal theoretical cross section. The band around these limits shows the effect of the PDF choice and of the variation of renormalisation/factorisation scale by a factor of two.

keep the slepton mass slightly above the $\chi_{2}^{0}$ mass which corresponds to a three-body decay.

\section{Squarks and gluinos}

\subsection{Generic $\tilde{q}$ and $\tilde{g}$ searches}

In $p \bar{p}$ collisions, squarks $(\tilde{q})$ and gluinos $(\tilde{g})$, the superpartners of quarks and gluons, are expected first to be abundantly produced if they are sufficiently light, and second to largely exceed the mass reach achieved at LEP. However, these searches have large background at the Tevatron. The final states are studied within the framework of mSUGRA assuming $R$-parity conservation. All SUSY particles, except the lightest neutralino, are unstable and will therefore decay into their SM counterparts right after being produced, leading to a cascade decay with a final state consisting of several jets from the squarks and the gluinos, plus missing transverse energy coming from the neutralinos. Note that to interpret the results of this search, the ten SUSY partners of the five light quarks flavors were considered to be degenerate in mass by $\mathrm{D} \varnothing$. In the following, the squark mass is therefore defined as the average mass of all squarks other than the superpartners of the top. The $\mathrm{CDF}$ analysis assumes that only the first and second generation masses are degenerated.

The most constraining direct limits on squark and gluino masses are published by the $\mathrm{D} \varnothing$ Collaboration

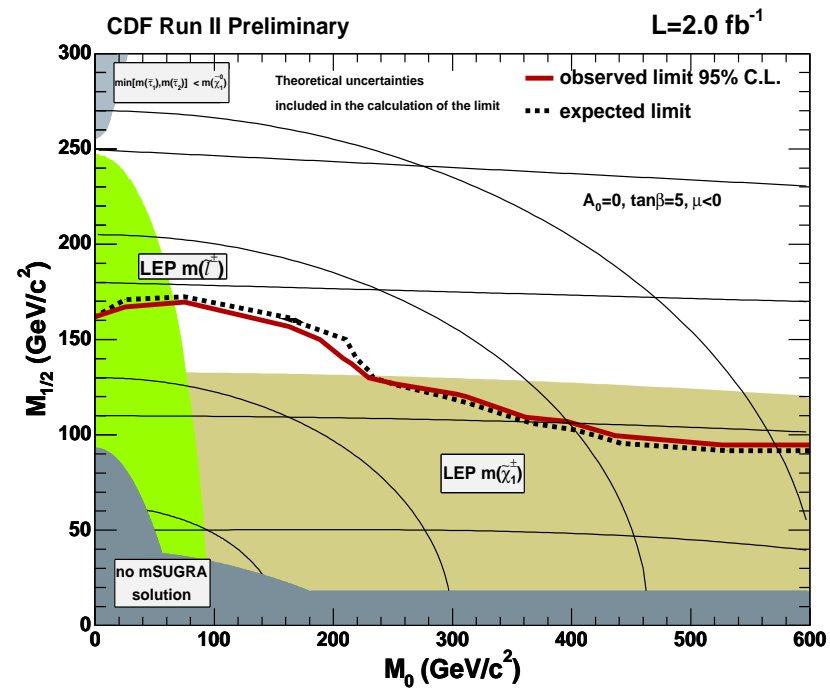

Fig. 34. Regions excluded by the squark and gluino analyses at the $95 \%$ C.L. in the $\left(m_{0}, m_{1 / 2}\right)$ plane, in the framework of mSUGRA assuming $R$-parity conservation, using $2 \mathrm{fb}^{-1}$ of CDF Run II data 118. The regions excluded by LEP chargino and slepton searches are shown. The nearly horizontal black lines are the iso-mass curves for gluinos corresponding to masses of 150, 300, 450 and $600 \mathrm{GeV}$. The other lines are iso-mass curves for squarks, corresponding to masses of 150, 300, 450 and $600 \mathrm{GeV}$.

[117, based on an analysis of $2.1 \mathrm{fb}^{-1}$ of data. A preliminary result with similar sensitivity was shown by CDF [118 during the winter 2008 conferences with $2 \mathrm{fb}^{-1}$ of data.

Three different scenarios have been probed by both $\mathrm{CDF}$ and $\mathrm{D} \varnothing$ experiments. The first one corresponds to pair production of squarks, each decaying into a quark and a neutralino $\left(\tilde{q} \rightarrow q \chi_{1}^{0}\right)$, leading to a two jets $+\mathscr{E}_{T}$ final state. This decay channel is dominant if the gluino is heavier than the squark $\left(m_{\tilde{q}} \ll m_{\tilde{g}}\right)$. The second scenario applies when the squark is heavier than the gluino, leading to a final state with 4 jets and $\not_{T}$ from $\tilde{g} \rightarrow \tilde{q}^{*} \bar{q} \rightarrow q \bar{q} \chi_{1}^{0}$. The third one addresses similar squark and gluino masses, with a final state of three or more jets arising from $\tilde{q} \tilde{g}$ associated production.

Table 3 from DØ illustrates the selection criteria used for these searches. The data show good agreement with SM expectations after requiring dedicated multijet $+E_{T}$ triggers and tight cuts on $\mathscr{E}_{T}$ and the scalar $p_{T} \operatorname{sum}\left(H_{T}\right)$. No signal is seen and cross section upper limits at $95 \%$ C.L. have been obtained for the sets of minimal SUGRA parameters considered $(\tan \beta=$ $5(3), A_{0}=0\left(-2 m_{0}\right), \mu<0$ for $\left.\mathrm{CDF}(\mathrm{D} \varnothing)\right)$. The two Collaborations show the results translated into the excluded regions in the $\left(m_{\tilde{g}}, m_{\tilde{q}}\right)$ and $\left(m_{0}, m_{1 / 2}\right)$ planes.

The observed and expected mass limits derived for $\mathrm{D} \varnothing$ using $2.1 \mathrm{fb}^{-1}$ are given in Fig. 33 as functions of the squark and gluino masses, improving on previous published limits. Lower limits at 95\% C.L. of $379 \mathrm{GeV}$ and $308 \mathrm{GeV}$ on the squark and gluino masses, respectively, are derived in the most conservative hy- 


\begin{tabular}{|c|c|c|c|}
\hline Preselection Cut & \multicolumn{3}{|c|}{ All Analyses } \\
\hline$E_{T}$ & \multicolumn{3}{|c|}{$\geq 40$} \\
\hline |Vertex $z$ pos.| & \multicolumn{3}{|c|}{$<\overline{6} 0 \mathrm{~cm}$} \\
\hline Acoplanarity & \multicolumn{3}{|c|}{$<165^{\circ}$} \\
\hline Selection Cut & $\tilde{q} \tilde{q} \rightarrow q \chi_{1}^{0} q \chi_{1}^{0}$ & $\tilde{q} \tilde{g} \rightarrow q \chi_{1}^{0} q \bar{q} \chi_{1}^{0}$ & $\tilde{g} \tilde{g} \rightarrow q \bar{q} \chi_{1}^{0} q \bar{q} \chi_{1}^{0}$ \\
\hline $\begin{array}{l}\text { Trigger } \\
\text { jet } p_{T}\end{array}$ & $\begin{array}{l}\text { dijet } \\
\geq 35\end{array}$ & $\begin{array}{c}\text { multijet } \\
\geq 35\end{array}$ & $\begin{array}{c}\text { multijet } \\
\geq 35\end{array}$ \\
\hline Electron veto & yes & $\overline{\text { yes }}$ & yes \\
\hline Muon veto & yes & yes & yes \\
\hline$\Delta \phi\left(\mathscr{E}_{T}\right.$, ,et $\left._{1}\right)$ & $\geq 90^{\circ}$ & $\geq 90^{\circ}$ & $\geq 90^{\circ}$ \\
\hline$\Delta \phi\left(\mathbb{E}_{T}\right.$, jet $\left._{2}\right)$ & $\geq 50^{\circ}$ & $\geq 50^{\circ}$ & $\geq 50^{\circ}$ \\
\hline$\Delta \phi_{\min }\left(\not \mathbb{E}_{T}\right.$, any jet $)$ & $\geq 40^{\circ}$ & - & - \\
\hline$H_{T}$ & $\geq 325$ & $\geq 375$ & $\geq 400$ \\
\hline $\mathscr{E}_{T}$ & $\geq 225$ & $\geq 175$ & $\geq 100$ \\
\hline$N_{\text {backgrd. }}$ & $11.1 \pm 1.2_{-2.3}^{+2.9}$ & $10.7 \pm 0.9_{-2.1}^{+3.1}$ & $17.7 \pm 1.1_{-3.3}^{+5.5}$ \\
\hline$N_{\text {sig. }}$ & $10.4 \pm 0.6_{-1.8}^{+1.8}$ & $12.0 \pm 0.7_{-2.3}^{+2.5}$ & $17.0 \pm 1.2_{-2.9}^{+3.3}$ \\
\hline$N_{\text {obs. }}$ & 11 & 9 & 20 \\
\hline
\end{tabular}

Table 3. Selection criteria for the three squark and gluino analyses published by the $\mathrm{D} \varnothing$ Collaboration 117 . with $2.1 \mathrm{fb}^{-1}$ of data (all energies and momenta in $\mathrm{GeV})$. |Vertex $z$ pos. $\mid$ is the longitudinal position of the interaction collision with respect to the detector center. The acoplanarity is defined as the azimuthal angle between the two leading jets. First and second (third and fourth) jets are also required to be central $|\eta|<0.8(|\eta|<2.5)$ with $p_{T} \geq 35 \mathrm{GeV}\left(p_{T} \geq 20 \mathrm{GeV}\right.$ for the fourth jet). The missing transverse energy and scalar $p_{T}$ sum are denoted $\mathbb{E}_{T}$ and $H_{T}$, respectively. The numbers of events observed and expected from SM backgrounds and from signal are given for each analysis (the first uncertainty is statistical and the second is systematic).

pothesis by $\mathrm{D} \emptyset$. The corresponding expected limits are $377 \mathrm{GeV}$ and $312 \mathrm{GeV}$. For the particular case $m_{\tilde{q}} \simeq m_{\tilde{g}}$, squark and gluino masses below $390 \mathrm{GeV}$ are excluded. The observed limit becomes $408 \mathrm{GeV}$ for the NLO nominal signal cross section computed with the CTEQ6.1M PDF [119] and for the renormalization and factorization scale $\mu_{r, f}=Q$, where $Q$ is taken to be equal to $m_{\tilde{g}}$ for $\tilde{g} \tilde{g}$ production, $m_{\tilde{q}}$ for $\tilde{q} \tilde{q}$, and $\left(m_{\tilde{g}}+m_{\tilde{q}}\right) / 2$ for $\tilde{q} \tilde{g}$ production. The factor of two on the renormalization and factorization scale reduces or increases the nominal signal cross sections by $15-20 \%$. The PDF and $\mu_{r, f}$ effects were added in quadrature to compute minimum and maximum signal cross sections. If one considers the less conservative scenario (maximum signal cross section), the observed lower mass limit for $m_{\tilde{q}} \simeq m_{\tilde{g}}$ is $427 \mathrm{GeV}$ at $95 \%$ C.L.

The CDF search with $2 \mathrm{fb}^{-1}$ excludes masses up to $392 \mathrm{GeV}$ in the region where gluino and squark masses are similar, gluino masses up to $280 \mathrm{GeV}$ for every squark mass, and gluino masses up to $423 \mathrm{GeV}$ for squark masses below $378 \mathrm{GeV}$. Figure 34 shows the results of this analysis translated into the excluded regions in the mSUGRA $\left(m_{0}, m_{1 / 2}\right)$ plane. This search improves on the limit from indirect LEP searches for $m_{0}$ values between 75 and $250 \mathrm{GeV}$ and for $m_{1 / 2}$ values between 130 and $170 \mathrm{GeV}$. However, the LEP Higgs search limits remain more constraining in a purely mSUGRA scenario [120.

The D $\varnothing$ and CDF limits use slightly different model parameters and methods to compute the excluded masses. Thus, they are not directly comparable. However, it was verified that similar results hold for a large class of parameter sets.

A complementary search for squarks has been performed by $\mathrm{D} \emptyset$ in the topology of multijet events accompanied by large missing transverse energy and at least one tau lepton decaying hadronically using $1 \mathrm{fb}^{-1}$ of data 121. Lower limits on the squark mass up to

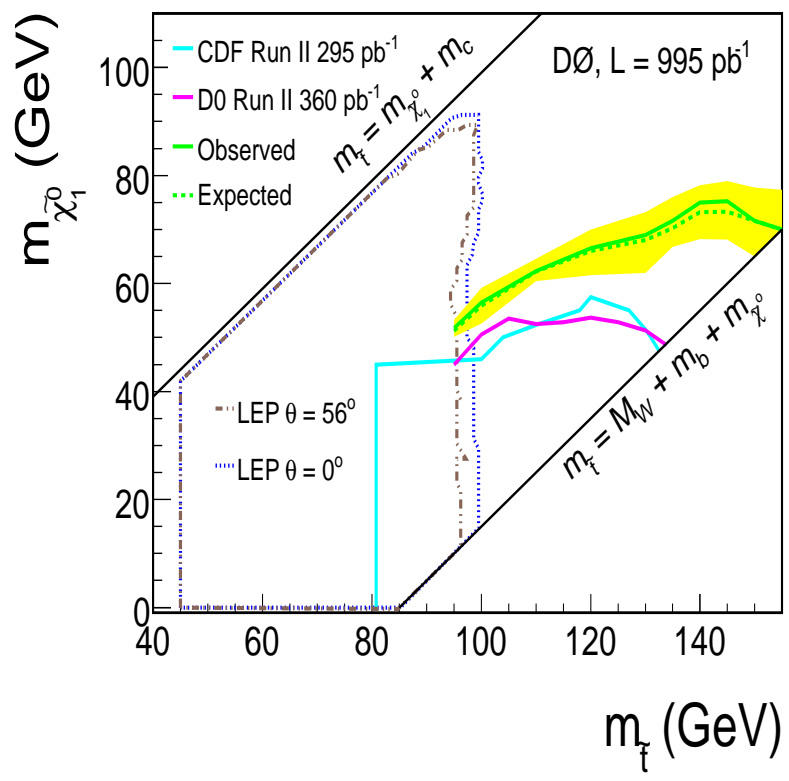

Fig. 35. DØ 95\% C.L. exclusion contours in the stop and neutralino mass plane, assuming a stop branching ratio of $100 \%$ into a charm quark and a neutralino. This search is based on $1 \mathrm{fb}^{-1}$ of data 122 .

$366 \mathrm{GeV}$ are derived in the framework of mSUGRA with parameters enhancing final states with taus. This analysis has the advantage of providing additional sensitivity for squark searches, mainly at large values of $\tan \beta$.

\subsection{Stop and sbottom searches}

For the third generation, mass unification is broken in many SUSY models due to potentially large mixing effects. This can result in a sbottom or stop with much 


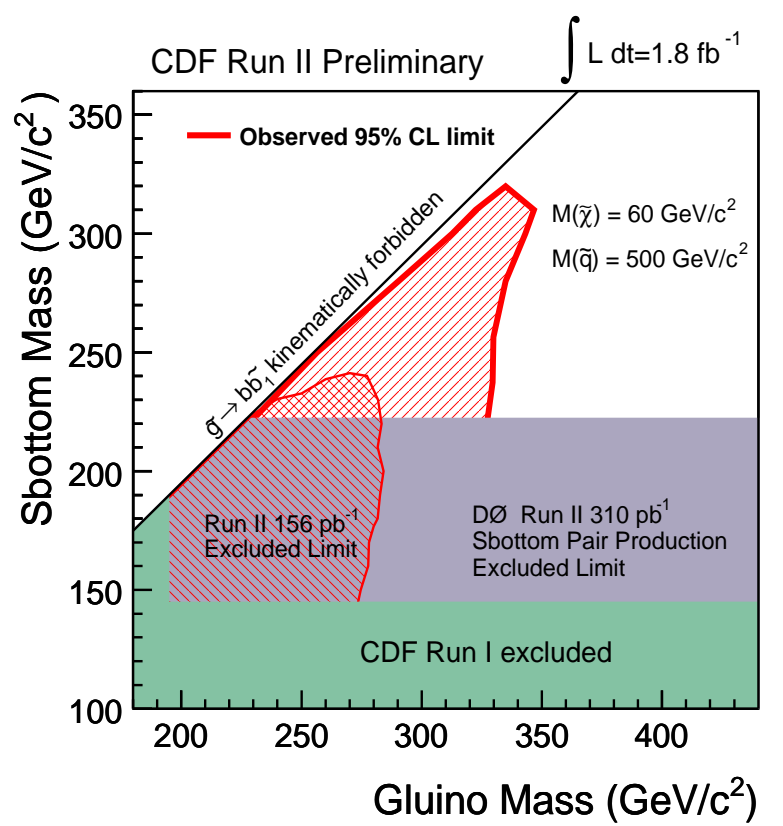

Fig. 36. CDF 95\% C.L. exclusion contours in the sbottom and neutralino mass plane, assuming gluino pair production where the gluino decays to $b \tilde{b}$ with subsequent sbottom decay to a $b$-quark and the lightest neutralino. This search is based on $1.8 \mathrm{fb}^{-1}$ of data 127 .

lower mass than the other squarks and gluinos. In addition, the lightest stop quark could well be the lightest of all quarks because of the impact of the large top Yukawa coupling on the renormalization group equations.

Dedicated searches are conducted in a general MSSM framework assuming the decays $\tilde{t} \rightarrow c \tilde{\chi}_{1}^{0}$ and $\tilde{b} \rightarrow$ $b \tilde{\chi}_{1}^{0}$ are the only ones kinematically allowed. Despite a much smaller cross section for $\tilde{t}$ and $\tilde{b}$ production compared to previous generic squark searches, heavy-flavor tagging can be used to reduce the important SM backgrounds. However, in the mass range of interest, the jets are much softer compared to the generic squark search, and therefore the QCD multijet background is much larger, reducing the stop and sbottom masses which can be excluded.

D $\varnothing$ has recently submitted for publication an update using $1 \mathrm{fb}^{-1}$ of data 122 compared to previous $\mathrm{D} \varnothing[123$ and CDF 124 published results with about $300 \mathrm{pb}^{-1}$ of the case where the stop decays with a branching ratio of $100 \%$ into a charm quark and a neutralino. Good agreement between the data and the SM prediction is obtained. The derived limits at $95 \%$ C.L. on the stop mass are shown in Fig. 35. With the theoretical uncertainty on the $\tilde{t}$ pair production cross section taken into account, the largest limit on $m_{\tilde{t}}$ is $150 \mathrm{GeV}$, for $m_{\chi_{1}^{0}}=65 \mathrm{GeV}$.

At large values of $\tan \beta$, the mixing can be enhanced in the sbottom sector. The analysis of the decay channel $\tilde{b} \rightarrow b \tilde{\chi}_{1}^{0}$ is similar to the one applied for

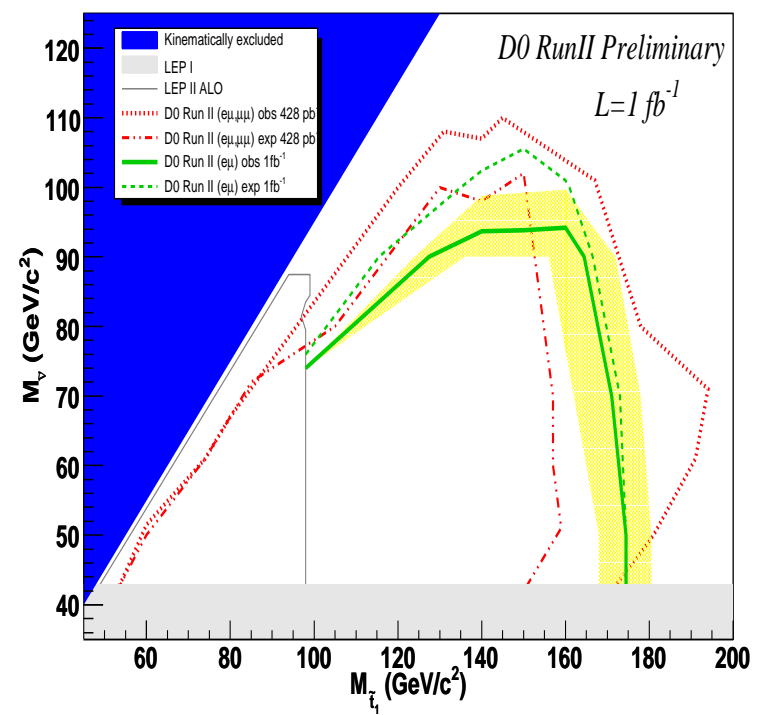

Fig. 37. DØ 95\% C.L. exclusion contours in the stop and sneutrino mass plane, assuming $\tilde{t} \rightarrow b \ell \tilde{\nu}$ and $\tilde{\nu} \rightarrow \nu \chi_{1}^{0}$ decay modes using $1 \mathrm{fb}^{-1}$ of data [130.

the stop except that higher masses can be excluded because heavy-flavor tagging is more efficient for $b$-jets than for $c$-jets. Supersymmetric bottom quark masses up to $193 \mathrm{GeV}$ for a neutralino mass of $40 \mathrm{GeV}$ are excluded by CDF with $295 \mathrm{pb}^{-1}$ of data [124. For the $\mathrm{D} \varnothing$ analysis [125] using $310 \mathrm{pb}^{-1}$, the maximum $m_{\tilde{b}}$ excluded is $222 \mathrm{GeV}$, which is the most restrictive limit on the sbottom mass to date from direct $\tilde{b}$ pair production.

The CDF Collaboration also considered the scenario where the sbottom could be produced through the decay of gluinos into bottom and sbottom quarks, yielding a signature consisting of four $b$-jets and two neutralinos from the sbottom decay $\tilde{b} \rightarrow b \tilde{\chi}_{1}^{0}$. Requiring inclusive double $b$-tagging, CDF observes 4 events where $2.6 \pm 0.7$ are expected in $156 \mathrm{pb}^{-1}$ of Run II data [126]. Exclusion lower limits have been published on the masses of the gluino and sbottom up to 280 and $240 \mathrm{GeV}$, respectively. This result has been recently updated with $1.8 \mathrm{fb}^{-1}$ of CDF data [127. At least one $b$-tagged jet was required and two different signal regions were optimized. In the small (large) $\Delta m=m_{\tilde{g}}-m_{\tilde{\chi}_{1}^{0}}$ region, 19 (25) events are observed for $22.0 \pm 3.6(22.7 \pm 4.6)$ events expected from SM processes. The $\left(m_{\tilde{b}}, m_{\tilde{g}}\right)$ exclusion contour plot at $95 \%$ C.L. is shown in Fig. 36. A lower gluino mass limit $m_{\tilde{g}}>340 \mathrm{GeV}$ is set for $m_{\tilde{b}}=300 \mathrm{GeV}, m_{\tilde{\chi}_{1}^{0}}=$ $60 \mathrm{GeV}$, and $m_{\tilde{q}}=500 \mathrm{GeV}$.

The D $\varnothing$ Collaboration has searched for a light stop in the lepton+jets channel using two scenarios. The first one uses the stop decay modes $\tilde{t}_{1} \rightarrow t \chi_{1}^{0} \rightarrow b W^{+} \chi_{1}^{0}$. In this analysis, kinematic differences between stop pair production and the dominant $t \bar{t}$ process are used to separate the two possible contributions. The preliminary results [128] with $1 \mathrm{fb}^{-1}$ set upper cross section 


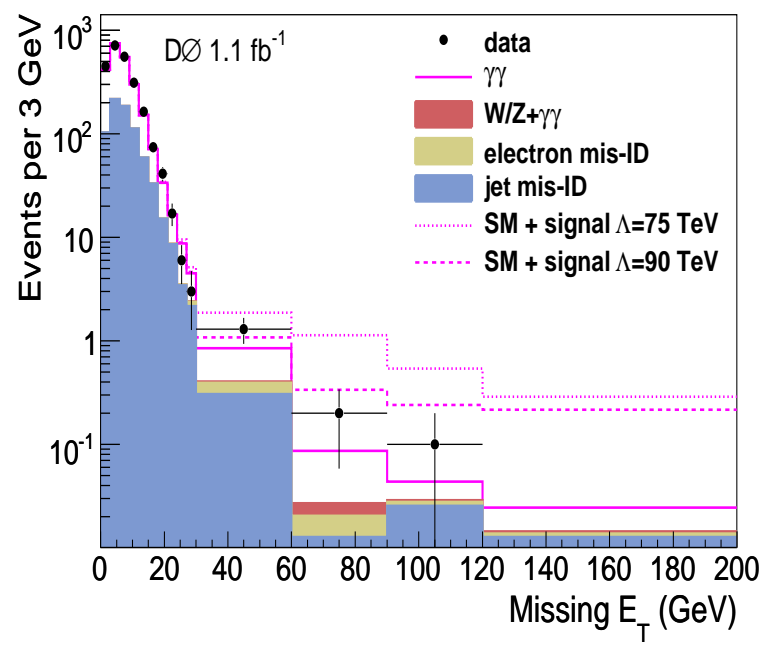

Fig. 38. $D \varnothing \mathbb{E}_{T}$ distribution in $1.1 \mathrm{fb}^{-1}$ of $\gamma \gamma$ data, along with expected background processes 139. The expected $\mathbb{E}_{T}$ distribution for GMSB SUSY signal with $\Lambda=75 \mathrm{TeV}$ and $90 \mathrm{TeV}$ are presented as dotted and dashed lines, respectively.

limits at $95 \%$ C.L. on $\tilde{t}_{1} \tilde{t}_{1}$ production that are a factor of about 7-12 higher than expected for the MSSM model for stop masses ranging between $145-175 \mathrm{GeV}$. The second scenario considers the pair production of the stop decaying into a $b$-quark and the supersymmetric partner of the neutrino,i.e., the sneutrino $(\tilde{\nu})$. This decay $\tilde{t} \rightarrow b \ell \tilde{\nu}$ is then followed by the $\tilde{\nu} \rightarrow \nu \chi_{1}^{0}$ involving only invisible particles. The ensuing final state consists of two leptons, two $b$-jets and missing transverse energy. The result combines $e \mu+$ jets and $\mu \mu+$ jets final states and limits are set in the plane $\left(m_{\tilde{t_{1}}}, m_{\tilde{\nu}}\right)$ as shown in Fig. [37. The published result [129] with $428 \mathrm{pb}^{-1}$ of data has been recently updated [130] with $1.1 \mathrm{fb}^{-1}$ and there is now good agreement between the expected and observed limits.

\section{Gauge mediated SUSY breaking}

In gauge mediated SUSY breaking models (GMSB), the gravitino, with a mass less than few $\mathrm{keV}$, is the LSP. The phenomenology of these models is therefore determined by the nature and the lifetime of the nextto-lightest supersymmetric particle (NLSP), which can be either a neutralino or the lightest stau, depending on the choice of model parameters 3 .

\subsection{Long-lived final state}

It is possible for a stau NLSP in these models to be longlived [131. Stau pair production has been searched for by DØ using $390 \mathrm{pb}^{-1}$ of data 132 . These long-lived particles loose energy principally by ionization and can traverse the entire detector, registering in the muon detectors. The search is not yet sensitive enough to set a stau lower limit. In anomaly mediated supersymmetry breaking (AMSB), or in models that do not have gaugino mass unification, the signature is the same for long-lived charginos that escape the detector 133 . The larger production cross section allows a preliminary lower limit of $140 \mathrm{GeV}$ on higgsino-like charginos and $174 \mathrm{GeV}$ on gaugino-like charginos, both at the $95 \%$ C.L.

\subsection{Diphoton final state}

Final states with two photons and $\mathbb{E}_{T}$ can be produced in GMSB models. In such a scenario, the lightest neutralino $\left(\chi_{1}^{0}\right)$ decays into a photon and a weakly interacting stable gravitino $(\tilde{G})$. Most of the searches assume the prompt decay $\chi_{1}^{0} \rightarrow \gamma \tilde{G}$. The CDF Collaboration, however, also searched in $570 \mathrm{pb}^{-1}$ of data for non-prompt decays and a $\chi_{1}^{0}$ with a lifetime that is on the order of nanoseconds or more [134. Two candidate events, consistent with the background estimate of $1.3 \pm 0.7$ events, are selected based on the arrival time of the photon at the calorimeter. This result allows for setting both quasi-model-independent cross section limits and for an exclusion region of GMSB models in the $\chi_{1}^{0}$ lifetime versus mass plane $\left(\tau_{\chi_{1}^{0}}, m_{\chi_{1}^{0}}\right)$, with a mass reach of $101 \mathrm{GeV}$ for $\tau_{\chi_{1}^{0}}=5 \mathrm{~ns}$.

As for the prompt decay $\chi_{1}^{0} \rightarrow \gamma \tilde{G}$, CDF [135] and $\mathrm{D} \varnothing$ [136] combined their published results based on 200-260 $\mathrm{pb}^{-1}$ of data. The combined limit [137] excludes a chargino mass of less than $209 \mathrm{GeV}$, for GMSB parameters following the "Snowmass benchmark scenario" [138]: messenger mass $m_{M}=2 \Lambda$ where $\Lambda$ is the effective SUSY-breaking scale, $\tan \beta=15, \mu>0$, and the number of messenger fields $N_{M}=1$. The DØ Collaboration recently published an update using $1.1 \mathrm{fb}^{-1}$ of data 139 . The $\mathbb{E}_{T}$ distribution for the $\gamma \gamma$ sample is given in Fig. 38 with the expected signal contribution for two different values of the effective energy scale $\Lambda$. After determination of all backgrounds from data, D $\varnothing$ observes no excess of such events and thus sets $95 \%$ C.L. limits: the masses of the lightest chargino and neutralino are found to be larger than $229 \mathrm{GeV}$ and $125 \mathrm{GeV}$, respectively. These results represent the most stringent limits to date on this particular GMSB SUSY model.

\section{$11 R$-parity violation}

The MSSM superpotential is minimal in the sense that it is sufficient to produce a phenomenologically viable model. However, the most general gauge-invariant and renormalizable superpotential would include additional terms like

$$
W_{\mathrm{RPV}}=\lambda_{i j k} L_{i} L_{j} \bar{E}_{k}+\lambda_{i j k}^{\prime} L_{i} Q_{j} \bar{D}_{k}+\lambda_{i j k}^{\prime \prime} \bar{U}_{i} \bar{D}_{j} \bar{D}_{k},
$$

where $i=1,2,3$ are the family indices. The lepton and quark doublet superfields (weak isospin singlet superfields) are denoted $L$ and $Q(E, U$ and $D)$, respectively. 


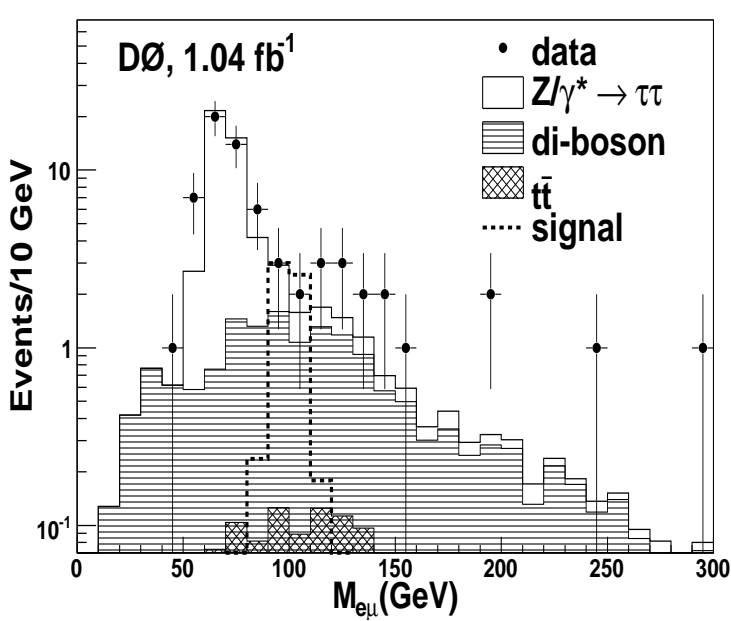

Fig. 39. Invariant mass of the electron-muon system in $1 \mathrm{fb}^{-1}$ of data collected by the D $\varnothing$ experiment 144 . The search is performed in the context of $R$-parity-violating production and decay. The dashed line indicates the signal hypothesis for a third-generation sneutrino $\left(\tilde{\nu}_{\tau}\right)$ with a mass of $100 \mathrm{GeV}$ and $\sigma \times B r$ of $0.057 \mathrm{pb}$.

These terms violate both baryon number (B) and lepton number $(\mathrm{L})$, which is in contradiction with experimental observations. The most obvious experimental constraint comes from the non-observation of proton decay, which would violate both B and L by 1 unit. Therefore, these new couplings in the trilinear terms, if present in nature, must be extremely small. In the MSSM, a new symmetry is thus introduced to eliminate the possibility of $\mathrm{B}$ and $\mathrm{L}$ violating terms in the superpotential. This new symmetry, called $R$-parity [3], is a multiplicatively conserved quantum number defined as $P=(-1)^{3(B-L)}$, which takes a value of +1 for SM particles and -1 for SUSY particles.

The CDF and D $\varnothing$ Collaborations have considered a number of scenarios under the hypothesis that $R$ parity violation (RPV) can occur. The experimental consequences are characterized by less missing transverse energy and more leptons and jets in the final states, due to the decay of the LSP into SM particles. In addition, sparticles may be resonantly produced by RPV couplings as single sparticles, by virtue of which the LSP cannot be a candidate for dark matter.

Searches for gaugino pair production via $\lambda_{121}$ and $\lambda_{122}$ with a signature of at least four charged leptons and two neutrinos are published by CDF 140 (DØ 141) using $346 \mathrm{pb}^{-1}\left(360 \mathrm{pb}^{-1}\right)$ of data. The case in which a $\tau$ appears in the final state via $\lambda_{133}$ coupling has been included in the $\mathrm{D} \varnothing$ analysis. Using the mSUGRA model with $m_{0}=1 \mathrm{TeV}, \tan \beta=5$, and $\mu>0$, D $\varnothing$ obtains $95 \%$ C.L. lower limits on the $\chi_{1}^{0}$ $\left(\chi_{1}^{ \pm}\right)$masses of $119,118,86 \mathrm{GeV}(231,229,166 \mathrm{GeV})$ for the corresponding $\lambda_{121}, \lambda_{122}$, and $\lambda_{133}$ couplings, respectively. For the $\mathrm{CDF}$ analysis, the $\chi_{1}^{0}$ mass limits range from 98 to $110 \mathrm{GeV}$, while the chargino mass limits range from 185 to $203 \mathrm{GeV}$ at $95 \%$ C.L., depending on the choice of model parameters.

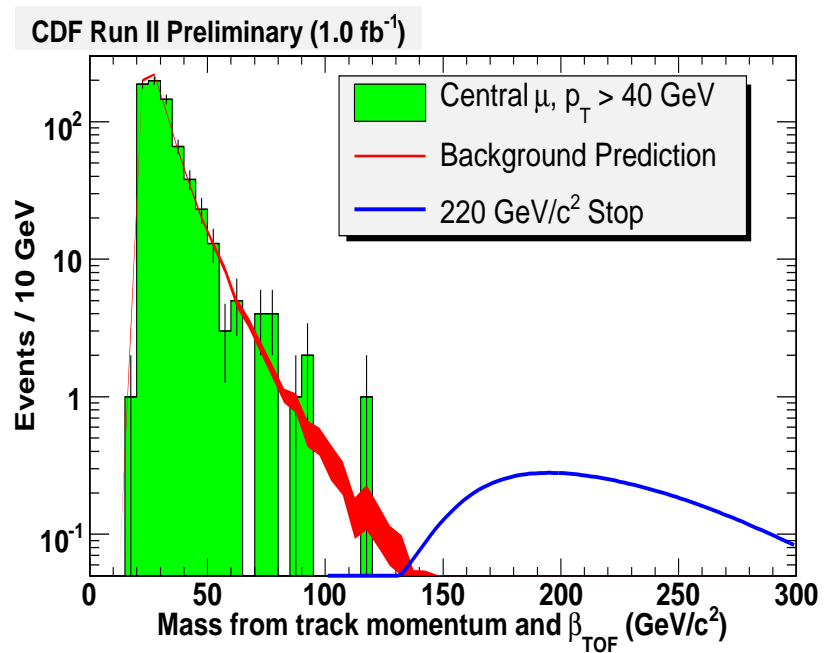

Fig. 40. Mass distribution measured in $1 \mathrm{fb}^{-1}$ of data by the time-flight flight and transverse momentum of tracks in events collected by the CDF experiment using the high transverse momentum muon trigger. The expected contribution from stable stop pair production is shown for a stop mass of $220 \mathrm{GeV} 153$.

A search for pair production of scalar top quarks decaying via the $R$-parity-violating $\lambda_{333}^{\prime}$ coupling to a $\tau$ lepton and a $b$ quark has been presented by the CDF Collaboration based on $322 \mathrm{pb}^{-1}$ of data 142 . A lower mass limit $m_{\tilde{t}_{1}}>151 \mathrm{GeV}$ has been set in the final state of either an electron or a muon from the first $\tau$ decay, a hadronic decay for the second $\tau$, and two or more jets.

Resonant slepton production has also been probed at the Tevatron. A single slepton could be produced in hadron collisions by $L Q D$ interactions followed by decays into SM di-lepton final states via $L L E$ interactions, leading to a high-mass di-lepton resonance. The CDF 143 and DØ 144 Collaborations have reported a search for resonant production of sneutrinos decaying into an electron and a muon using $344 \mathrm{pb}^{-1}$ and $1 \mathrm{fb}^{-1}$ of data, respectively. The invariant mass of the electron-muon system in the $\mathrm{D} \varnothing$ search is shown in Fig. 39. For a sneutrino with mass of $100 \mathrm{GeV}$, $\lambda_{311}^{\prime}>1.6 \times 10^{-3}$ is excluded by D $\varnothing$ at $95 \%$ C.L. when the $\lambda_{312}$ coupling constant is fixed at 0.01 . CDF excludes $\lambda_{311}^{\prime}$ values above 0.01 for a $\tilde{\nu}_{\tau}$ mass of $300 \mathrm{GeV}$ and $\lambda_{132}>0.02$. In addition, CDF published sneutrino mass limits depending on the $\lambda^{\prime}$ and $\lambda$ couplings considered in the other $e e, \mu \mu, \tau \tau$ final states based on $\approx 200 \mathrm{pb}^{-1}$ [145, 146].

Results from D $\varnothing$ have been published [147] based on $380 \mathrm{pb}^{-1}$ on the production and decay of resonant smuons and muon-sneutrinos in the channels $\tilde{\mu} \rightarrow$ $\tilde{\chi}_{1}^{0} \mu, \tilde{\mu} \rightarrow \tilde{\chi}_{2,3,4}^{0} \mu$, and $\tilde{\nu}_{\mu} \rightarrow \tilde{\chi}_{1,2}^{ \pm} \mu$. A lower limit on the slepton mass $m_{\tilde{l}} \leq 363 \mathrm{GeV}$ is set for $\lambda_{211}^{\prime} \geq 0.10$, $\tan \beta=5, A_{0}=0$ and $\mu<0$. 


\section{Long-lived particles}

Although cosmological considerations put strict limits on new particles that are absolutely stable, these restrictions do not apply to particles that live long enough to decay outside the detector [148. Several models, outlined below, predict charged or neutral longlived particles decaying inside or outside the detector.

\subsection{Neutral long-lived particles}

The existence of neutral long-lived particles decaying into two leptons that arise from a highly displaced vertex is expected in "hidden valley" theories [149] or SUSY models with $R$-parity violation [3. Motivated by the excess of di-muon events observed by the Fermilab neutrino experiment $\mathrm{NuTeV}$ [150, D $\varnothing$ has published [151] a search based on $380 \mathrm{pb}^{-1}$ of data assuming a benchmark model where the $\chi_{1}^{0}$ has traveled at least $5 \mathrm{~cm}$ and decays via RPV to $\mu^{+} \mu^{-} \nu$. The background has been estimated to be about one event and, since no candidates were observed, a 95\% C.L. upper cross section limit of $0.14 \mathrm{pb}$ is set on pair-production of neutral long-lived particles with a mass of $10 \mathrm{GeV}$ and a lifetime of $4 \times 10^{-11}$.

\subsection{Charged long-lived particles}

If a long-lived particle has a large mass and is charged (CHAMP) 152, it will appear in the detector as a slowly moving, highly ionizing particle with large transverse momentum that can be observed in the muon detectors. CDF has performed a model independent search by measuring the time-of-flight of particles from muon triggers in $1 \mathrm{fb}^{-1}$ of data [153. As shown in Fig. 40, the result is consistent with muon background expectation. Within the context of stable stop pair production, CDF infers an upper mass limit of $250 \mathrm{GeV}$ at $95 \%$ C.L.

The introduction of a fourth generation quark $b^{\prime}$ provides another possibility for such long-lived particles [154. The CDF Collaboration reported [155] a result, based on $193 \mathrm{pb}^{-1}$ of data, for such particles using $Z$ boson decays to muons and reconstructing dimuon vertices in the tracker. $\mathrm{D} \varnothing$ also performed a $b^{\prime}$ search, using the capability of its detector to reconstruct the direction of electromagnetic showers, and thus enhancing its sensitivity to long-lived particles. Although limits have been set within the framework of the $b^{\prime}$ model, loose requirements are imposed to limit potentially model-dependent selection. No evidence of such excess is found in $1 \mathrm{fb}^{-1}$ of data by $\mathrm{D} \varnothing[156$. Limits are set on the production cross section and lifetime of such long-lived particles that decay into a $Z$ boson or any final state with a pair of electrons or photons with mass above $75 \mathrm{GeV}$ at $95 \%$ C.L.

In a variant of SUSY known as split supersymmetry [157], gluino decays into squarks and the neutralino LSP are suppressed, leading to a long lived gluino. At the Tevatron, such colorless bound states (R-hadrons)

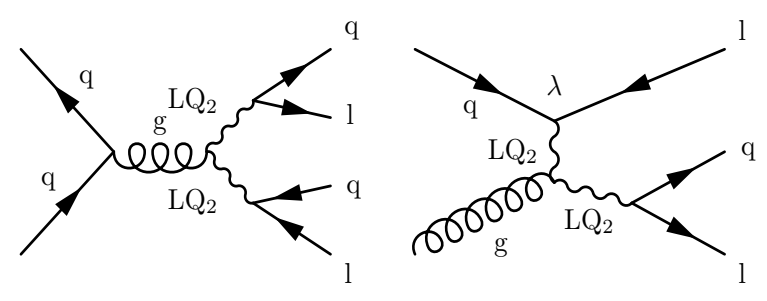

Fig. 41. Examples of leading-order Feynman graphs for pair-production (left) and single production (right) of leptoquarks.

of a gluino and other quarks or gluons could be pair produced through strong interactions. As studied in Ref. [158, some charged R-hadrons have the potential to become "stopped gluinos", by losing all of their momentum through ionization and come to rest in the calorimeter. No excess is observed above the primary source of background coming from cosmic muons in $410 \mathrm{pb}^{-1}$ of $\mathrm{D} \varnothing$ data $[159$. Their main decay mode is expected to be $\tilde{g} \rightarrow g \chi_{1}^{0}$ with a lifetime assumed to be long enough such that the decay of the gluino occurs during a bunch-crossing adequately later than the one which has produced it (about $30 \mu \mathrm{s}$ ). Limits are therefore placed on the gluino cross section times the stopping probability as a function of the gluino and $\chi_{1}^{0}$ masses, for gluino lifetimes from $30 \mu$ s to 100 hours. This analysis excludes $m_{\tilde{g}}<270 \mathrm{GeV}$ for a $\chi_{1}^{0}$ mass of $50 \mathrm{GeV}$, assuming a $100 \%$ branching fraction for $\tilde{g} \rightarrow g \chi_{1}^{0}$, a gluino lifetime less than 3 hours, and a neutral to charged R-hadron conversion cross section of $3 \mathrm{mb}$.

\section{Leptoquarks}

Leptoquarks $(L Q)$ are colored bosons that were postulated to explain the parallels between the families of quarks and leptons [160. They are predicted in many extensions of the standard model, such as $\mathrm{SU}(5)$ grand unification [161, superstring [162], and compositeness models 163. Figure 41 shows mechanisms for leptoquark production and decay in $p \bar{p}$ collisions, where leptoquarks can be pair produced via the strong interaction. Single leptoquark production can also occur in association with a lepton.

\subsection{LQ pair production}

At the Tevatron, $L Q$ states would be predominately pair produced with larger cross sections predicted for vector (spin 1) than for scalar (spin 0) leptoquarks. They are expected to decay into a quark and a charged lepton with a branching fraction $\beta$, or into a quark and a neutrino with a branching fraction $(1-\beta)$. Experimental limits on lepton number violation, on flavorchanging neutral currents, and on proton decay motivate the assumption that there would be three different generations of leptoquarks, where each leptoquark 
generation couples to only one generation of quarks and leptons.

Results have been published by CDF for first generation scalar leptoquarks $\left(L Q_{1}\right)$ using about $200 \mathrm{pb}^{-1}$ of data. No evidence is observed of such particles in the topologies arising from $L Q_{1} \overline{L Q}_{1} \rightarrow$ eqeq, $L Q_{1} \overline{L Q}_{1} \rightarrow$ $e q \nu q$, and $L Q_{1} \overline{L Q}_{1} \rightarrow q \nu q \nu$ [164,165]. Lower mass limits are derived: 236,205 and $145 \mathrm{GeV}$ for $\beta=1$, $\beta=0.5$ and $\beta=0.1$, respectively. The $L Q_{1}$ mass limits which are published in the eqeq and eqvq final states by $\mathrm{D} \varnothing$ with $252 \mathrm{pb}^{-1}$ of data are 256 and $234 \mathrm{GeV}$, for $\beta=1$ and 0.5 , respectively 166 . In $L Q_{1} \overline{L Q}_{1} \rightarrow q \nu q \nu, \mathrm{D} \varnothing$ has published [167] a result with $310 \mathrm{pb}^{-1}$ and a lower mass limit of $136 \mathrm{GeV}$ is set at the $95 \%$ C.L. Recently, CDF has released a search based on $2 \mathrm{fb}^{-1}$ of dijet $+\mathbb{E}_{T}$ data 168 . Two separate analyses are performed. The first one requires two jets with $p_{T}>30 \mathrm{GeV}$, no third jet with $p_{T}>15 \mathrm{GeV}$, $E_{T}>80 \mathrm{GeV}$, and $H_{T}>125 \mathrm{GeV}$. The second one search in the high kinematic region defined by $H_{T}>$ $225 \mathrm{GeV}$ and $E_{T}>100 \mathrm{GeV}$. In both regions, CDF compares the expected SM backgrounds with data and no excess is observed. A scalar leptoquark model is used to place a limit of $m \overline{L Q}_{1}>177 \mathrm{GeV}$, for $\beta=0$ at $95 \%$ C.L.

Second generation scalar leptoquarks $\left(L Q_{2}\right)$ have also been searched for at the Tevatron. The CDF Collaboration has published a result in the dimuons + jets and muon+missing energy + jets topologies using 198 $\mathrm{pb}^{-1}$ of data 169. Combining the results with those from the $\not_{T}+$ jets channel topology [165], CDF excludes $L Q_{2}$ with masses below $226 \mathrm{GeV}$ for $\beta=1$, $208 \mathrm{GeV}$ for $\beta=0.5$, and $143 \mathrm{GeV}$ for $\beta=0.1$ at $95 \%$ C.L. The D $\varnothing$ Collaboration has published limits in the channel $L Q_{2} \overline{L Q}_{2} \rightarrow \mu q \mu q$ using an integrated luminosity of $294 \mathrm{pb}^{-1}$ [170. In combination with previous D $\varnothing$ measurements, lower mass limits of $m_{L Q_{2}}>251 \mathrm{GeV}$ for $\beta=1$ and $m_{L Q_{2}}>204 \mathrm{GeV}$ for $\beta=0.5$ are set. The first D $\varnothing$ search performed in Run II in the channel $L Q_{2} \overline{L Q}_{2} \rightarrow \mu q \nu q$, which has maximal sensitivity for $\beta=0.5$, is based on $1 \mathrm{fb}^{-1}$ of data 171 . From this analysis alone, a lower mass limit for scalar second generation leptoquarks of $m_{L Q_{2}}>214 \mathrm{GeV}$ at $\beta=0.5$ is set at $95 \%$ C.L. Using $2 \mathrm{fb}^{-1}$ of dijet $+\mathbb{E}_{T}$ data, CDF excludes $L Q_{2}$ masses below $177 \mathrm{GeV}$ at $95 \%$ C.L [168].

A search for third generation scalar $L Q_{3}$ pair production has been performed in the $\tau b \tau b$ channel using $1 \mathrm{fb}^{-1}$ of data collected at D $\varnothing \sqrt{172}$. To increase the search sensitivity, advantage is taken of the presence of heavy-flavor jets in the signal. No evidence of signal has been observed, and limits are set on the production cross section as a function of the leptoquark mass. Assuming $\beta$, the branching fraction of the leptoquark into $\tau b$, equal to 1 , the limit on the mass is $180 \mathrm{GeV}$ at $95 \%$ C.L. With a smaller dataset of $0.4 \mathrm{fb}^{-1}$, assuming a decay into $b \nu$, the limit is $229 \mathrm{GeV}[173$. If leptoquark decays into a $\tau$ lepton and a top quark are taken into account, and if equal couplings are assumed, a mass limit of $m_{L Q_{3}}>221 \mathrm{GeV}$ is set by D $\varnothing$ at $95 \%$ C.L. 173 . The CDF Collaboration has performed a similar analysis with $322 \mathrm{pb}^{-1}$ of data

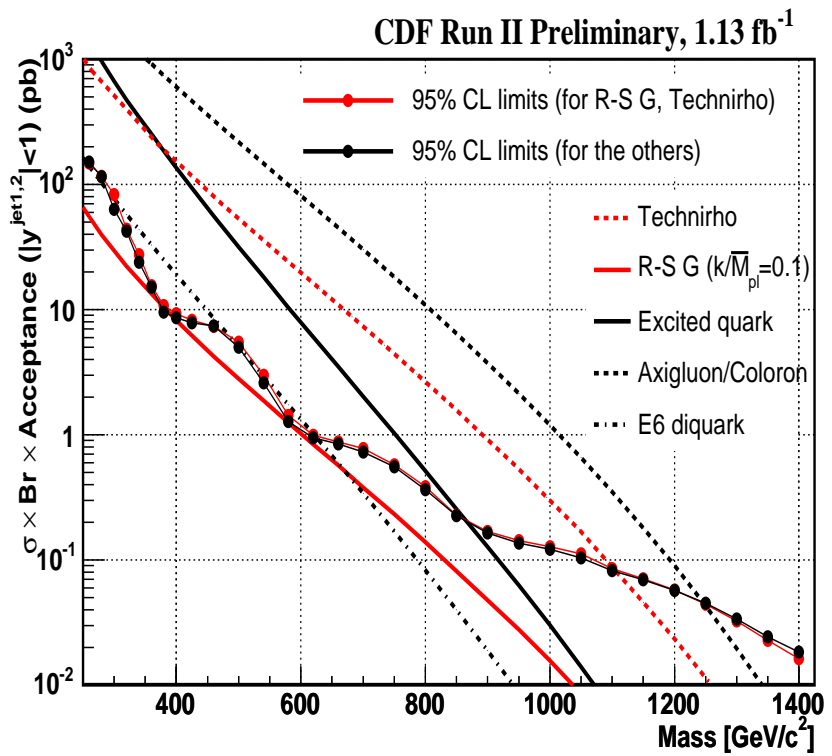

Fig. 42. CDF 95\% C.L. limits based on $1.1 \mathrm{fb}^{-1}$ of data 183 on the Randall-Sundrum graviton $(G \rightarrow q \bar{q}, g g \quad[184)$, color-octet techni-rho production $\left(\rho_{T} \rightarrow q \bar{q}, g g\right.$ [185]), excited quark $\left(q^{*} \rightarrow q g\right.$ [181]), axigluon and flavor-universal coloron $(A \rightarrow q \bar{q}$ [186]), and $E_{6}$ di-quark $\left(D\left(D^{c}\right) \rightarrow(q q) \bar{q} \bar{q}\right.$ 162] $)$, compared with the theoretical predictions for production of these particles.

but in the context of vector leptoquarks $\left(V L Q_{3}\right)$. Assuming Yang-Mills (minimal) couplings, CDF obtaines the most stringent upper limit on the $V L Q_{3}$ pair production cross section of $344 \mathrm{fb}(493 \mathrm{fb})$ and lower limit on the $V L Q_{3}$ mass of $317 \mathrm{GeV}(251 \mathrm{GeV})$ at $95 \%$ C.L in $\tau b$ decay 174 . Finally, a mass limit of $m \overline{L Q}_{3}>167 \mathrm{GeV}$ is set for third generation of scalar leptoquark using $2 \mathrm{fb}^{-1}$ of CDF dijet $+\mathbb{E}_{T}$ data [168]. In this case, the efficiency for third generation events to pass a dijet plus missing $E_{T}$ selection criteria is smaller due to lepton rejection requirements, and therefore the mass limits set are lower than those for the first and second generation.

\subsection{Single LQ production}

The production of single leptoquarks leads to final states consisting of two leptons and one jet. The D $\varnothing$ Collaboration has published a search in the $\mu \mu j$ final state using $300 \mathrm{pb}^{-1}$ of data 175 . Compared to the search for leptoquarks which considered only pairproduction, the mass limits are improved to $m_{L Q}>$ $274 \mathrm{GeV}$ for $\beta=1$ and $\lambda=1$, where $\lambda$ is the leptoquarklepton-quark coupling. For $\beta=0.5$, a lower limit on the mass of a second generation scalar leptoquark $m_{L Q}$ $>226 \mathrm{GeV}$ is set at $95 \%$ C.L.

\section{Compositeness}

In the SM, the quarks and leptons are treated as fundamental particles. However, one proposed explanation 
for the three generations is a compositeness model of the known leptons and quarks [176].

\subsection{Excited lepton}

Compositeness models comprise a large spectrum of excited states. The CDF and D $\varnothing$ Collaborations have searched for excited electron $\left(e^{*}\right)$ in the process $p \bar{p} \rightarrow e^{*} e$, with the $e^{*}$ subsequently decaying to an electron plus photon. The agreement observed by $\mathrm{D} \varnothing$ in $1 \mathrm{fb}^{-1}$ 177 and CDF in $202 \mathrm{pb}^{-1} 178$ of data with the SM backgrounds are interpreted in the context of a model that describes production by four-fermion contact interactions (CI) and excited electron decay via electroweak processes. Choosing the scale for CI to be $\Lambda=1 \mathrm{TeV}$, $e^{*}$ masses below $756 \mathrm{GeV}$ are excluded at $95 \%$ C.L. by the $\mathrm{D} \varnothing$ analysis. To make a comparison with LEP results, CDF also reinterprets its search in the gauge-mediated model and excludes $126 \mathrm{GeV}<m_{e^{*}}<430 \mathrm{GeV}$ at $95 \%$ C.L. for the phenomenological coupling $f / \Lambda \approx 10^{-2} \mathrm{GeV}$ [178.

Similarly, searches for excited muons $\left(\mu^{*}\right)$ subsequently decaying to a muon plus photon have been carried out in a data sample corresponding to an integrated luminosity of $371 \mathrm{pb}^{-1}$ for CDF 179 and 380 $\mathrm{pb}^{-1}$ for $\mathrm{D} \varnothing$ 180. CDF excludes in the contact interaction model $107 \mathrm{GeV}<m_{\mu^{*}}<853 \mathrm{GeV}$ for $\Lambda=m_{\mu^{*}}$ and in the gauge-mediated model $100 \mathrm{GeV}<m_{\mu^{*}}<$ $410 \mathrm{GeV}$ for $f / \Lambda \approx 10^{-2} \mathrm{GeV}$ at $95 \%$ C.L. Choosing the scale for contact interactions to be $\Lambda=1 \mathrm{TeV}$, masses below $618 \mathrm{GeV}$ are excluded by the $\mathrm{D} \varnothing$ search.

\subsection{Excited quark}

The $\mathrm{D} \varnothing$ Collaboration has published a search within the framework of a quark substructure model [181. In $370 \mathrm{pb}^{-1}$ of data, no indication for resonances in the $Z$ +jet channel has been observed, where the $Z$ boson is detected via its $Z \rightarrow e^{+} e^{-}$decay mode. This analysis leads to a mass limit $m_{q^{*}}>510 \mathrm{GeV}$ at 95\% C.L., assuming the decay mode $q^{*} \rightarrow q+Z$ for the excited quark [182. The CDF preliminary result based on $1.1 \mathrm{fb}^{-1}$ of data excludes the mass region $260 \mathrm{GeV}<m_{q^{*}}<870 \mathrm{GeV}$ at $95 \%$ C.L. assuming the decay mode $q^{*} \rightarrow q g$ [183]. The obtained limits are shown in Fig. 42 for various models corresponding to the production of new particles that decay into dijet.

In the same vein, CDF searched for new particles that lead to a $Z$ boson plus jets but, this time, in the context of a fourth generation model [154]. In a data sample of $1 \mathrm{fb}^{-1}$, the $Z$ boson decays to $e e$ and $\mu \mu$ are used to set a lower limit on $b^{\prime}$ quark masses below $268 \mathrm{GeV}$ at $95 \%$ C.L., assuming the decay mode $b^{\prime} \rightarrow b+Z$ [187].

In $1.9 \mathrm{fb}^{-1}$ of lepton+jet data, CDF also investigated the existence of a massive gluon and set limits on the coupling strength of this particle as function of its mass 188. A search for the heavy top $\left(t^{\prime}\right)$ quark pair production decaying to $W q$ final states in $2.3 \mathrm{fb}^{-1}$

\section{CDF Run II Preliminary}

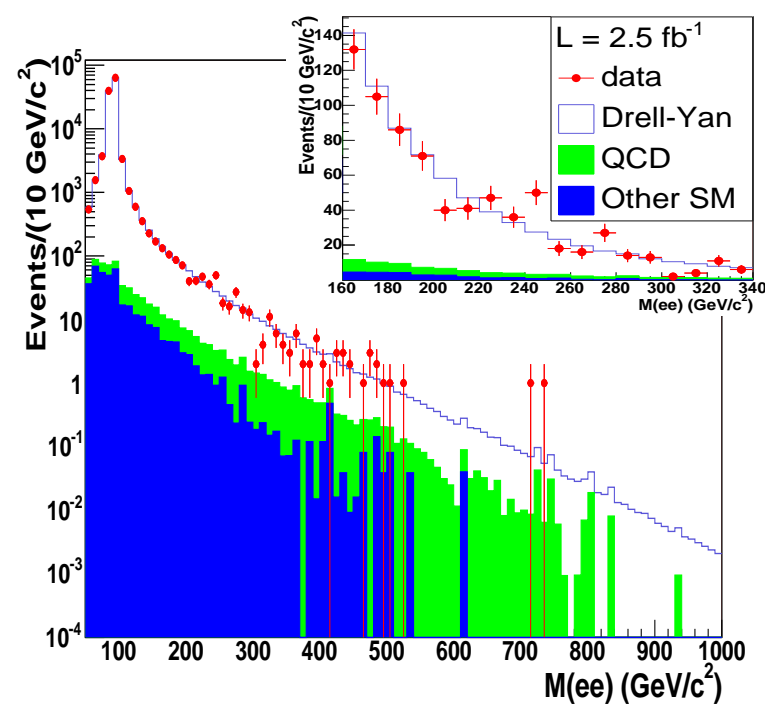

Fig. 43. The dielectron mass measured by CDF and the expected background in $2.5 \mathrm{fb}^{-1}$ of data 192. A slight excess in the data is observed in the region $m_{e^{+} e^{-}} \approx$ $240 \mathrm{GeV}$.

of CDF lepton+jets data excludes a fourth-generation $t^{\prime}$ quark with a mass below $284 \mathrm{GeV}$ at $95 \%$ C.L [189].

\section{Extra gauge bosons}

Multiple extensions of the SM predict extra gauge bosons. For instance, $Z^{\prime}$ are predicted in $E_{6}$ GUTs models [190, and $W^{\prime}$ bosons appear in models such as left-rightsymmetric theories [191. The new gauge group can comprise a new mixing angle and new couplings depending on the models considered.

\section{$15.1 Z^{\prime}$ bosons}

The CDF Collaboration has recently released a new preliminary result in the search for dielectron resonances using $2.5 \mathrm{fb}^{-1}$ of data 192 . The previous CDF searches have been published with $0.2-1.3 \mathrm{fb}^{-1}$ of data 145, 193 194. Resonance with dilepton in the final states have always been leading channels for early discovery searches due to low backgrounds. In addition, lepton energy and momentum can be measured precisely by combining calorimeter and tracking information. The searches are performed by reconstructing the dielectron mass, as shown in Fig. 43. The $Z$ mass peak and the Drell-Yan tail at high mass is well reproduced by the SM background prediction. However, in the region $m_{e^{+} e^{-}} \approx 240 \mathrm{GeV}$, an excess of data over background of $3.8 \sigma$ is observed, with a $0.6 \%$ probability that it is caused by the background fluctuation, given that the search probes the mass range $150 \mathrm{GeV}-1 \mathrm{TeV}$. A typical di-electron event is displayed in Fig. 44. 
By performing a scan for high-mass resonances, CDF sets limits depending on the model considered. For instance, a lower mass limit of $966 \mathrm{GeV}$ can be set assuming SM-like couplings of the $Z^{\prime}$, with a somewhat lower mass limit for $E_{6} Z^{\prime}$ bosons with masses below $737 / 933 \mathrm{GeV}$ (lightest/heaviest) GeV excluded at $95 \%$ C.L. The DØ Collaboration has presented a preliminary results 195] with $200 \mathrm{pb}^{-1}$ of data and excludes lower masses for $Z^{\prime}$ boson with SM-like couplings to fermions of $780 \mathrm{GeV}$ at $95 \%$ C.L.

The $e^{+} e^{-}$final state can also easily be reinterpreted in the context of technicolor models, which predict large amounts of techniparticle production at the Tevatron [196]. Degenerated technihadrons $\left(\rho_{T}, \omega_{T}\right)$ decaying into $e^{+} e^{-}$are excluded for certain model parameters with masses below $367 \mathrm{GeV}$, based on 200 $\mathrm{pb}^{-1}$ of DØ data [197.

Similar analyses in the dimuon channel have been carried out by CDF based on $200 \mathrm{pb}^{-1}$ of data 145 and by $\mathrm{D} \varnothing$ with $250 \mathrm{pb}^{-1}$ of data 198 . The signal process $Z^{\prime} \rightarrow \tau^{+} \tau^{-}$has been searched by CDF with $195 \mathrm{pb}^{-1}$ of data and a lower mass limit of $399 \mathrm{GeV}$ (SM couplings) has been set [146].

A general search for resonances decaying to a neutral $e \mu$ final state based on $344 \mathrm{pb}^{-1}$ of CDF data has been interpreted in the context of lepton family number violating (LFV) $Q_{12}^{l}$ couplings [199] of the $Z^{\prime}$ boson and $E_{6}$-like models of $U(1)^{\prime}$ symmetry [200].

A search for a narrow-width heavy resonances decaying into top quark pairs $X \rightarrow t \bar{t}$ based on $913 \mathrm{pb}^{-1}$ has been submitted for publication by D $\varnothing 201$. This result was recently updated with $2.1 \mathrm{fb}^{-1}$ of data for the winter 2008 conferences [202]. Within a topcolorassisted technicolor model 203, the existence of a leptophobic $Z^{\prime}$ boson with mass $m_{Z^{\prime}}<760 \mathrm{GeV}$ and width $\Gamma_{Z^{\prime}}=0.012 m_{Z^{\prime}}$ are excluded at $95 \%$ C.L. Similarly, CDF performed several searches for a $t \bar{t}$ resonance in the lepton+jets final state using $680 \mathrm{pb}^{-1}$ [204] and $1 \mathrm{fb}^{-1}$ 205] of data. A leptophobic $Z^{\prime}$ predicted by the topcolor theory is excluded below $725 \mathrm{GeV}$.

\section{$15.2 W^{\prime}$ bosons}

Both CDF 206] and DØ 207] have published results of searches for a heavy charged vector boson $\left(W^{\prime}\right)$ decaying to an electron-neutrino pair using $205 \mathrm{pb}^{-1}$ and $1 \mathrm{pb}^{-1}$ of data, respectively. In the context of a $W^{\prime}$ with SM coupling to fermions, and for these data samples, the lower mass limits are $1 \mathrm{TeV}$ for $\mathrm{D} \varnothing$ and $788 \mathrm{GeV}$ for $\mathrm{CDF}$, at $95 \%$ C.L. In a similar analysis that exploits $1.1 \mathrm{fb}^{-1}$ of dijet data, CDF excludes at $95 \%$ C.L. the existence of $W^{\prime}$ in the mass range $280<m_{W^{\prime}}<840 \mathrm{GeV}$ [183].

$W^{\prime}$ bosons that couple to right-handed fermions may not be able to decay to leptonic final states if the corresponding right-handed neutrinos $\left(\nu_{R}\right)$ are too massive. In this case, only hadronic decays are possible. To investigate the possibility of $W^{\prime}$ decaying into $t \bar{b}, \mathrm{D} \varnothing$ and CDF have used a similar approach as for

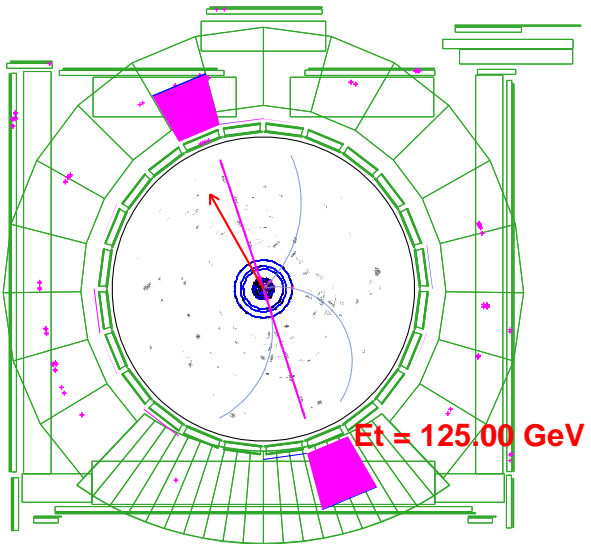

Fig. 44. Event display of a typical dielectron event, as measured by CDF in Run II 192 .

their single top searches. The $\mathrm{D} \emptyset$ Collaboration has published a result using $230 \mathrm{pb}^{-1}$ of data which excludes masses between $200 \mathrm{GeV}$ and $610 \mathrm{GeV}$ for a $W^{\prime}$ boson SM couplings 208. This search has been updated with approximately $0.9 \mathrm{fb}^{-1}$ of data 209 . For a lefthanded $W^{\prime}$ boson with SM couplings, DØ sets a lower mass limit of $731 \mathrm{GeV}$. For right-handed $W^{\prime}$ bosons, the lower mass limits on this hypothetical new particle at $95 \%$ C.L. are $739 \mathrm{GeV}$ assuming that the $W^{\prime}$ boson decays to both leptons and quarks, and $768 \mathrm{GeV}$ if it decays only to quarks. Assuming SM couplings to fermions for the $W^{\prime}$, CDF has used $1.9 \mathrm{fb}^{-1}$ of data to set limits of $m_{W^{\prime}}<800 \mathrm{GeV}$ when $m_{W^{\prime}}>m_{\nu_{R}}$ and $m_{W^{\prime}}<825 \mathrm{GeV}$ when $m_{W^{\prime}}<m_{\nu_{R}}$, at $95 \%$ C.L., on $W^{\prime}$ resonances in the $t \bar{b}$ decay channel 210].

\section{Large extra dimensions}

Models postulating the existence of large extra spatial dimensions have been proposed to solve the hierarchy problem posed by the large difference between the electroweak symmetry breaking scale at $1 \mathrm{TeV}$ and the Planck scale, at which gravity is expected to become strong.

In the original compactified large extra dimensions model of Arkani-Hamed, Dimopoulos and Dvali (ADD [211]), the effect of the extra spatial dimensions is visible as the presence of a series of quantized energy states referred to as graviton $(G)$ states Kaluza-Klein (KK) towers. However, the visible states are too close in mass to be distinguished individually and the coupling is small. Thus, it is only due to their very large number that the Kaluza-Klein gravitons could be observed. The direct production of gravitons, which immediately disappear into bulk space, gives rise to an excess of events with a high transverse energy jet (or photon) and large missing transverse energy. 


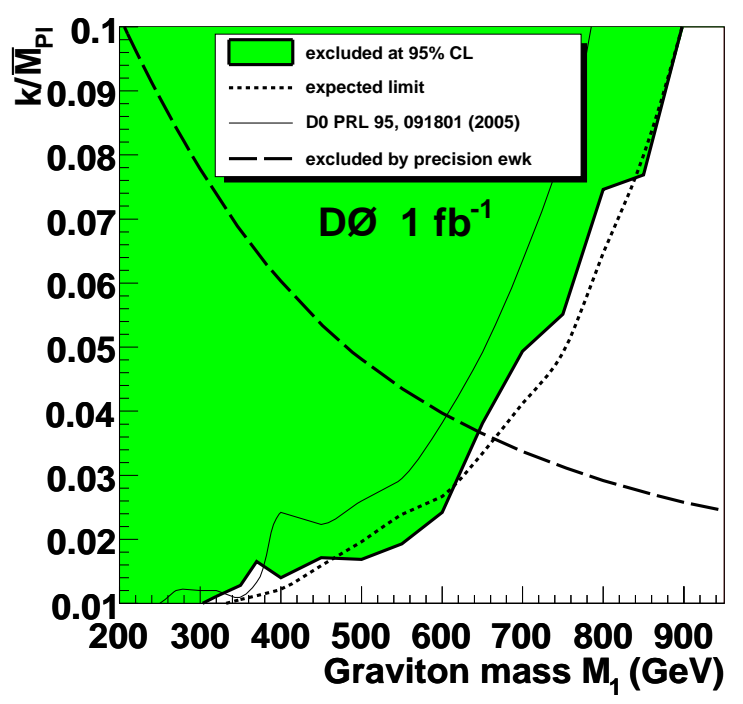

Fig. 45. Dø $95 \%$ C.L. upper limit on $k / M_{\mathrm{Pl}}$ versus graviton mass from $1 \mathrm{fb}^{-1}$ of data for the $e e+\gamma \gamma$ final states combined 214.

Another way to look for extra dimensions is to search for a resonance. The first excited graviton mode predicted by the Randall and Sundrum (RS) model [184 could be resonantly produced at the Tevatron. The graviton is then expected to decay to fermionanti-fermion or diboson pairs.

\subsection{Graviton resonances}

The CDF and D $\varnothing$ Collaborations have searched for resonances in their data in many different final states. Since the graviton has spin 2 , the branching fraction to the diphoton final state is expected to be twice that of $e^{+} e^{-}$final states. The diphoton background is estimated from misidentified electromagnetic objects and is extracted from the data. Results have been published by CDF 145] (DØ 212) based on $200 \mathrm{pb}^{-1}(260$ $\mathrm{pb}^{-1}$ ) of data. The combined $e e+\gamma \gamma$ final states have been recently published by both experiments based on 1.2-1.3 fb $\mathrm{fb}^{-1}$ of CDF 194 213] (DØ 214]) data. Limits obtained are as a function of the graviton mass and the coupling parameter $\left(k / M_{P l}\right)$, as represented in Fig. 45 for $\mathrm{D} \varnothing$. The CDF Collaboration derives a lower limit of $889 \mathrm{GeV}$ on the graviton mass at the $95 \%$ C.L. for $k / M_{P l}=0.1$. The $\mathrm{D} \varnothing$ combined result of both ee and $\gamma \gamma$ channels set lower masses limits of 300 (900) GeV at $95 \%$ C.L. for $k / M_{P l}=0.01(0.1)$ [214].

Recently, CDF has released a new result based on $1.1 \mathrm{fb}^{-1}$ of data in the search for a massive object decaying to a pair of $\mathrm{Z}$ bosons, both of which decay to $e e$ 215. The cross section times branching fraction for RS gravitons that decay to $Z$ bosons is small, leading to about one $G \rightarrow Z Z \rightarrow$ eeee expected event produced in $2 \mathrm{fb}^{-1}$ of data. For this analysis, CDF relaxed the lepton identification requirements to optimize the signal sensitivity. The relaxed selection ad-

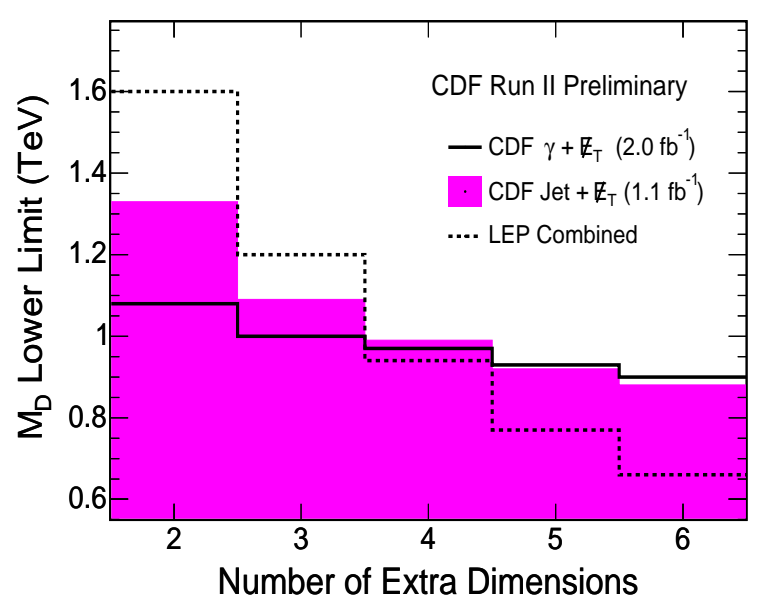

Fig. 46. Limits on the fundamental Planck scale $M_{D}$ for various numbers of extra dimensions from the CDF $q \bar{q} \rightarrow \gamma+G$ and $q \bar{q} \rightarrow$ jets $+G$ [219, along with the LEP limit.

mits more background, which is then rejected by imposing kinematic requirements on the invariant masses of the two $Z$ boson candidates. Finally, a sample in data that is kinematically similar to the signal has been used to estimate backgrounds in the signal region. No event are observed with $m_{\text {eeee }}>500 \mathrm{GeV}$ for an expected background of $0 \pm 0.02$ in $1.1 \mathrm{fb}^{-1}$ of data. The search is not yet sensitive to RS gravitons, so cross section limits of $\sigma \times B r(G \rightarrow Z Z \rightarrow$ eeee $) \lesssim 4 \mathrm{pb}$ for $500<m_{G}<800 \mathrm{GeV}$ are set on graviton production, assuming RS couplings.

\section{$16.2 \mathrm{Jet} / \gamma+\mathbb{E}_{T}$}

At the Tevatron, gravitons can be produced recoiling against a quark or a gluon jet 216, leading to an excess of events with a high $p_{T}$ jet and large $\mathbb{E}_{T}$. The resulting topology is a monojet. Similarly, gravitons can be produced directly in processes such as $q \bar{q} \rightarrow \gamma+G$.

The D $\varnothing$ Collaboration has investigated KK graviton production with a photon and missing transverse energy in $1 \mathrm{fb}^{-1}$ of data 217. At the $95 \%$ C.L., D sets limits on the fundamental Planck scale $\left(M_{D}\right)$ from $884 \mathrm{GeV}$ to $778 \mathrm{GeV}$ for 2 to 8 extra dimensions.

The CDF Collaboration published results based on $368 \mathrm{pb}^{-1}$ of monojet data 218 . Recently, the Collaboration has released a new result based on up to $2 \mathrm{fb}^{-1}$ of data which combines the jet $/ \gamma+\mathbb{E}_{T}$ final states 219 . The optimization for the ADD model yields a photon requirement of $E_{T}>90 \mathrm{GeV}$ with $E_{T}>50 \mathrm{GeV}$ and a jet requirement of $p_{T}>150 \mathrm{GeV}$ with $\mathbb{E}_{T}>150 \mathrm{GeV}$. The dominant SM background to the monojet search consists of $Z$ or $W$ boson plus jet production, with the $Z$ decaying to a pair of neutrinos, or the lepton from $W$ decay escaping detection. From the absence of an excess in the data, limits on $M_{D}>1.42(0.95)$ 


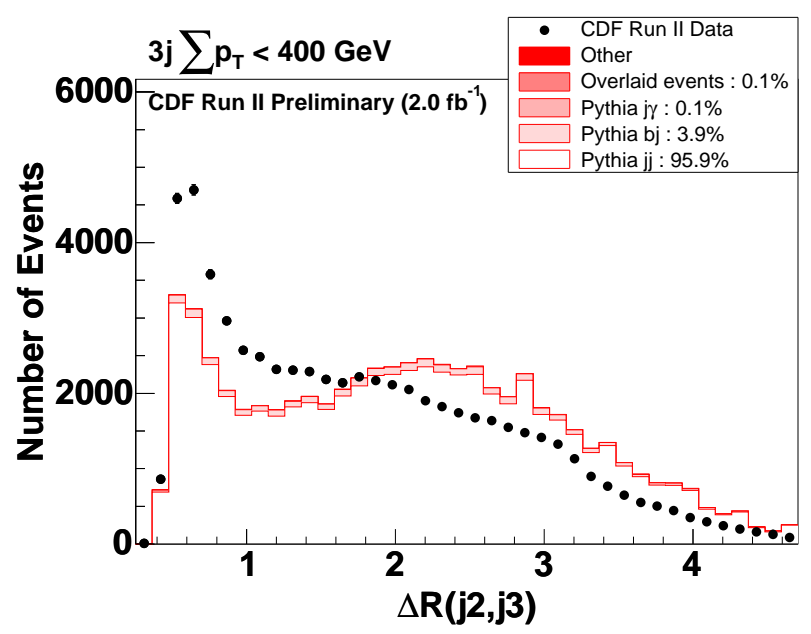

Fig. 47. CDF signature-based search using $2 \mathrm{fb}^{-1}$ of data. The distribution illustrates an example of a shape discrepancy found by VISTA 229 in the final state consisting of exactly three jets with $|\eta|<2.5$ and $p_{T}>17 \mathrm{GeV}$, and with one of the jets satisfying $|\eta|<1$ and $p_{T}>40 \mathrm{GeV}$. This distribution illustrates the effect underlying most of the VISTA shape discrepancies which were attributed to modeling parton radiation rather than to new physics.

are derived at $95 \%$ C.L. for the number of extra dimensions $n_{D}=2(6)$. The results are shown in Fig. 46 .

The D $\varnothing$ Collaboration has searched for monojet in $85 \mathrm{pb}^{-1}$ of data 220 . The most recent $\mathrm{D} \varnothing$ search is in the $q \bar{q} \rightarrow \gamma+G$ final state with $1 \mathrm{fb}^{-1}$ of data 221 . This analysis sets limits at $95 \%$ C.L. on $M_{D}$ from $884 \mathrm{GeV}$ to $778 \mathrm{GeV}$ for 2 to 8 extra dimensions.

\section{CDF signature-based searches}

Most of the searches presented so far have been optimized for signatures within a specific BSM model. However, it is also important to search for discrepancies with SM prediction in a model-independent approach, instead of focusing only on particular new physics scenarios.

Examples of such searches have been performed by the CDF Collaboration in the channels $\gamma \gamma+X$, where $X$ could be an electron, a muon, a photon, a tau, or missing transverse energy 222, 223], or $\ell \gamma+\mathscr{E}_{T}$,224, 225 . Other preliminary results have been presented for final state such as $\ell+\gamma+\mathbb{E}_{T}+b$ [226], or $Z$-boson $+X+$ $Y+$ anything, where $X$ and $Y$ can be leptons, photons, missing energy, or large total transverse energy 227 . These searches are based on $0.3-2.0 \mathrm{fb}^{-1}$ of data and nothing striking has been observed yet. In particular, the $\mathbb{E}_{T}$ +photon+lepton final state analysis [225] using $1 \mathrm{fb}^{-1}$ of Run II data has not confirmed the Run I event 228 .

An even more global analysis of CDF Run II data has also been carried out to search for indications of new phenomena in $2 \mathrm{fb}^{-1}$ of data 229]. First, a modelindependent approach (VISTA) focuses on obtaining a panoramic view of the entire data landscape, and is sensitive to new large-cross-section physics [230. It consists of a standard set of object identification criteria, which are used to identify isolated and energetic objects produced in the hard collision. All objects are required to have $p_{T}>17 \mathrm{GeV}$. Events are partitionated into exclusive final states labelled according to the objects $\left(e^{ \pm}, \mu^{ \pm}, \tau^{ \pm}, \gamma, j, b, \mathbb{E}_{T}\right)$ and compared to SM prediction. The SM prediction is obtained with PYTHIA 28 for the generation of inclusive $W, Z, \gamma \gamma$, $\gamma j, j j, W W, W Z$, and $Z Z$ production, while MADEVENT 231 provides events modeling for $W / Z+n$ jets and HERWIG [33 is used for top quark pair production. Detector response is modelled with the CDF simulation and a global fit for corrections factors (such as efficiencies and fake rates) is performed on the data. In the end, the number of events observed supports the standard model prediction with few discrepancies (see Fig. 47) attributed to modeling the parton radiation and underlying event in the data. A subset of the VISTA comparison is given in Table. 4.

A quasi-model-independent approach (SLEUTH) emphasizes the high- $p_{T}$ tails and is particularly sensitive to new electroweak-scale physics 232. SLEUTH is a quasi-model-independent search technique based on the assumption that new electroweak-scale physics will manifest itself as an excess of data over the SM expectation in a particular final state at large summed scalar transverse momentum $\left(\sum p_{T}\right)$. An algorithm has also been developed to search invariant mass distributions for "bumps" that could indicate resonant production of new particles. Here again, this global search for new physics in $2 \mathrm{fb}^{-1}$ of $p \bar{p}$ collisions reveals no indication of physics beyond the SM.

These global searches are complementary to targeted searches with specific signatures. However, it has been demonstrated that in order to exploit the data sample fully in term of sensitivity for a specific model with particularly distinct kinematic features, targeted searches out-perform these global approaches. For instance, a $115 \mathrm{GeV}$ SM Higgs boson decaying to two $b$-tagged jets in association with a heavy electroweak gauge boson is better treated using the $b \bar{b}$ invariant mass resonance rather than using the scalar transverse momentum sum.

\section{Conclusion}

The Tevatron Run II collider program is scheduled to run until October 2009 and possibly extend into 2010 to add an extra $25 \%$ of data, leading to an expected delivered integrated luminosity of about 8-9 $\mathrm{fb}^{-1}$. The search for the Higgs boson and physics beyond the standard model will greatly benefit from this additional integrated luminosity. The accelerator performance is excellent and provides a great opportunity for the $\mathrm{CDF}$ and $\mathrm{D} \varnothing$ experiments to meet or exceed their stated physics goals. Both CDF and DØ experiments have now surpassed the $4 \mathrm{fb}^{-1}$ in delivered luminosity.

While the ATLAS and CMS experiments at the LHC should be in good position to discover the Higgs boson on a time scale similar to the one considered at 
Table 4. A subset of the model-independent search (VISTA), which compares CDF Run II data with the SM prediction in $2 \mathrm{fb}^{-1}$ of data 229 . Events are partitioned into exclusive final states based on standard CDF particle identification criteria. Final states are labelled in this table according to the number and types of objects present, and are ordered according to decreasing discrepancy between the total number of events expected and the total number observed in the data. Only statistical uncertainties on the background prediction have been included in this Table.

CDF Run II Preliminary $\left(2.0 \mathrm{fb}^{-1}\right)$

The calculation of $\sigma$ accounts for the trials factor

\begin{tabular}{|c|c|c|c|c|c|c|c|c|c|c|c|}
\hline \multirow{3}{*}{ Final State } & \multirow{3}{*}{ Data } & \multirow{3}{*}{ Background } & \multirow[b]{3}{*}{$\sigma$} & \multirow[b]{2}{*}{ Final State } & \multirow[b]{2}{*}{ Data } & \multirow[b]{2}{*}{ Background } & \multirow[b]{2}{*}{$\sigma$} & \multicolumn{2}{|c|}{ Final State Data } & \multicolumn{2}{|l|}{ Background } \\
\hline & & & & & & & & & 32 & $32.2 \pm 10.9$ & \\
\hline & & & & $2 \mathrm{j} p$ high $-\Sigma p_{T}$ & 87 & $80.9 \pm 6.8$ & 0 & $\mathrm{j} \mu^{ \pm}$ & 14 & $11.5 \pm 2.6$ & \\
\hline$b e^{ \pm} p$ & 690 & $817.7 \pm 9.2$ & -2.7 & $2 \mathrm{j} p$ low $-\Sigma p_{T}$ & 114 & $79.5 \pm 100.8$ & 0 & $j \mu \pm \mu^{\mp}$ & 4852 & $4271.2 \pm 185.4$ & \\
\hline $\begin{array}{c}\gamma \tau^{ \pm} \\
+\end{array}$ & 1371 & $1217.6 \pm 13.3$ & +2.2 & $2 \mathrm{j} p \tau_{+}^{ \pm}$ & 18 & $13.2 \pm 2.2$ & 0 & $\mathrm{j} \mu \pm$ & 77689 & $76987.5 \pm 930.2$ & \\
\hline$\mu^{ \pm} \tau^{ \pm}$ & 63 & $\begin{array}{r}35.2 \pm 2.8 \\
327.80\end{array}$ & +1.7 & $2 \mathrm{j} \gamma \tau^{ \pm}$ & 142 & $144.6 \pm 5.7$ & 0 & $e^{ \pm} 4 \mathrm{j} p$ & 903 & $830.6 \pm 13.2$ & \\
\hline $\begin{array}{l}\text { b2jph high- } \Sigma p_{T} \\
2 \mathrm{j} \tau^{ \pm} \text {low }-\Sigma p_{T}\end{array}$ & 255 & $\begin{array}{l}327.2 \pm 8.9 \\
670.3+8.6\end{array}$ & $\begin{array}{l}-1.7 \\
-1.5\end{array}$ & $\begin{array}{l}2 \mathrm{j} \gamma \not p \\
2 \mathrm{j} \gamma\end{array}$ & $\begin{array}{r}908 \\
71364\end{array}$ & $\begin{array}{c}980.3 \pm 63.7 \\
73021.4 \pm 595.9\end{array}$ & $\begin{array}{l}0 \\
0\end{array}$ & $e^{ \pm} 4 \mathrm{j} \gamma$ & 25 & $29.2 \pm 3.6$ & \\
\hline & $\begin{array}{l}574 \\
148\end{array}$ & $\begin{array}{l}670.3 \pm 8.6 \\
199.8 \pm 5.2\end{array}$ & $\begin{array}{l}-1.0 \\
-1.4\end{array}$ & ${ }_{2 \mathrm{j} \mu}^{2 \mathrm{j} \gamma} \pm_{\tau} \mp$ & 16 & $19.3 \pm 2.2$ & 0 & $e^{ \pm} 4 \mathrm{j}$ & 15750 & $16740.4 \pm 390.5$ & \\
\hline $2 \mathrm{j} \tau^{ \pm} \tau^{\mp}$ & 33 & $62.1 \pm 4.3$ & -1.3 & $2 \mathrm{j} \mu^{ \pm} \gamma p$ & 31 & $27.7 \pm 7.7$ & 0 & $e^{ \pm} 3 \mathrm{j} p$ & 4054 & $4077.2 \pm 63.6$ & J \\
\hline$e^{ \pm} j$ & 741710 & $764832 \pm 6447.2$ & -1.3 & $2 \mathrm{j} \mu^{ \pm} \gamma$ & 57 & $58.2 \pm 13$ & 0 & $e^{ \pm}$ & 108 & $79.3 \pm 5$ & \\
\hline $\mathrm{j} 2 \tau^{ \pm}$ & 105 & $150.8 \pm 6.3$ & -1.2 & $2 \mathrm{j} \mu^{ \pm} \mu_{\not p}$ & 11 & $7.8 \pm 2.7$ & 0 & $e^{ \pm}$ & 60725 & $60409.3 \pm 723.3$ & \\
\hline$e^{ \pm} 2 j$ & 256946 & $249148 \pm 2201.5$ & +1.2 & $2 \mathrm{j} \mu^{ \pm} \mu^{\mp}$ & 956 & $924.9 \pm 61.2$ & 0 & $e^{ \pm}$ & 41 & $34.2 \pm 2.6$ & J \\
\hline $2 \mathrm{bj}$ low $-\Sigma p_{T}$ & 279 & $352.5 \pm 11.9$ & -1.1 & $2 \mathrm{j} \mu^{ \pm}$ & 22461 & $23111.4 \pm 366.6$ & 0 & & 37 & $47.2 \pm 2.2$ & J \\
\hline $\mathrm{j} \tau^{ \pm}$low- $\Sigma p_{T}$ & 1385 & $1525.8 \pm 15$ & -1.1 & $2 e^{ \pm} \mathrm{j}$ & 14 & $13.8 \pm 2.3$ & 0 & $e^{ \pm}$ & 109 & $95.9 \pm 6.8$ & U \\
\hline $2 \mathrm{~b} 2 \mathrm{j} \mathrm{low}-\Sigma p_{T}$ & 108 & $153.5 \pm 6.8$ & -1 & $2 e^{ \pm} e^{\mp}$ & 20 & $17.5 \pm 1.7$ & 0 & $e^{ \pm}$ & 25725 & $25403.1 \pm 209.4$ & U \\
\hline$\mu^{ \pm} \gamma \not p$ & 523 & $611 \pm 12.1$ & -0.8 & $2 \mathrm{~b}$ high- $\Sigma p_{T}$ & 666 & $689 \pm 9.4$ & 0 & $e^{ \pm}$ & 398 & $342.8 \pm 15.7$ & 0 \\
\hline $2 \mathrm{~b} \gamma$ & 108 & $70.5 \pm 7.9$ & +0.1 & $2 \mathrm{~b} \mathrm{low}-\Sigma p_{T}$ & 323 & $313.2 \pm 10.3$ & 0 & $e^{ \pm} 2 \mathrm{j} \mu \mp \not p$ & 22 & $14.8 \pm 1.9$ & 0 \\
\hline $8 \mathrm{j}$ & 14 & $13.1 \pm 4.4$ & 0 & $2 \mathrm{~b} 3 \mathrm{j} 1 \mathrm{ow}-\Sigma p_{T}$ & 53 & $57.4 \pm 6.5$ & 0 & $e^{ \pm}{ }_{2 j \mu} \mp$ & 23 & $15.8 \pm 2$ & 0 \\
\hline $7 \mathrm{j}$ & 103 & $97.8 \pm 12.2$ & 0 & $2 \mathrm{~b} 2 \mathrm{j}$ high $-\Sigma p_{T}$ & 718 & $803.3 \pm 12.7$ & 0 & $e^{ \pm}$ & 437 & $387 \pm 5.3$ & 0 \\
\hline $6 \mathrm{j}$ & 653 & $659.7 \pm 37.3$ & 0 & $2 \mathrm{~b} 2 \mathrm{j} p$ high $-\Sigma p_{T}$ & 15 & $21.8 \pm 2.8$ & 0 & $e^{ \pm}$ & 1333 & $1266 \pm 12.3$ & 0 \\
\hline $5 \mathrm{j}$ & 3157 & $3178.7 \pm 67.1$ & 0 & $2 \mathrm{~b} 2 \mathrm{j} \gamma$ & 32 & $39.7 \pm 6.2$ & 0 & $e^{ \pm}$ & 109 & $106.1 \pm 2.7$ & 0 \\
\hline $\begin{array}{l}4 \mathrm{j} \text { high }-\Sigma p_{T} \\
4 \mathrm{j} \text { low }-\Sigma p_{T}\end{array}$ & $\begin{array}{l}88546 \\
14872\end{array}$ & $\begin{array}{l}89096.6 \pm 935.2 \\
14809.6 \pm 186.3\end{array}$ & $\begin{array}{l}0 \\
0\end{array}$ & $2 \mathrm{~b} 2 \mathrm{j} \mu^{ \pm} p$ & 14 & $17.3 \pm 1.9$ & 0 & $e^{ \pm}$ & 960826 & $956579 \pm 3077.7$ & 0 \\
\hline $4 \mathrm{j} 2 \gamma$ & 46 & $46.4 \pm 3.9$ & 0 & & 22 & $21.8 \pm 2$ & 0 & $e^{ \pm} \gamma p$ & 497 & $496.8 \pm 10.3$ & 0 \\
\hline $4 \mathrm{j} \tau^{ \pm}$high $-\Sigma p_{T}$ & 29 & $26.6 \pm 1.7$ & 0 & $\begin{array}{l}2 \mathrm{~b} \mu^{\perp} \not \\
2 \mathrm{bj} \text { high- } \Sigma p_{T}\end{array}$ & $\begin{array}{r}11 \\
891\end{array}$ & $\begin{array}{c}14.4 \pm 2.1 \\
967.1 \pm 13.2\end{array}$ & $\begin{array}{l}0 \\
0\end{array}$ & $e^{ \pm}$ & 3578 & $3589.9 \pm 24.1$ & 0 \\
\hline $4 \mathrm{j} \tau^{ \pm} 1 \mathrm{ow}-\Sigma p_{T}$ & 43 & $63.1 \pm 3.3$ & 0 & $2 \mathrm{bj} p$ high $-\Sigma p_{T}$ & $\begin{array}{r}091 \\
25\end{array}$ & $31.3 \pm 3.1$ & 0 & $e^{ \pm} \mu^{ \pm} \not p$ & 31 & $29.9 \pm 1.6$ & 0 \\
\hline $4 \mathrm{j} p$ high $-\Sigma p_{T}$ & 1064 & $1012 \pm 62.9$ & 0 & $2 \mathrm{bj} \gamma$ & 71 & $54.5 \pm 7.1$ & 0 & $e^{ \pm} \mu^{\mp} \not p$ & 109 & $99.4 \pm 2.4$ & 0 \\
\hline $4 \mathrm{j} \gamma \tau^{ \pm}$ & 19 & $10.8 \pm 2$ & 0 & $2 \mathrm{bj} \mu^{ \pm} \not p$ & 12 & $10.7 \pm 1.9$ & 0 & $e^{ \pm} \mu^{ \pm}$ & 45 & $28.5 \pm 1.8$ & 0 \\
\hline $4 \mathrm{j} \gamma \not p$ & 62 & $104.2 \pm 22.4$ & 0 & $2 \mathrm{~b} e^{ \pm} 2 \mathrm{j} p$ & 30 & $27.3 \pm 2.2$ & 0 & $e^{ \pm} \mu^{\mp}$ & 350 & $313 \pm 5.4$ & 0 \\
\hline $4 \mathrm{j} \gamma$ & 7962 & $8271.2 \pm 245.1$ & 0 & $2 \mathrm{be} e^{ \pm j}$ & 72 & $66.5 \pm 2.9$ & 0 & $e^{ \pm} \mathrm{j} 2 \gamma$ & 13 & $16.1 \pm 3.9$ & 0 \\
\hline $4 \mathrm{j} \mu^{ \pm} p$ & 574 & $590.5 \pm 13.6$ & 0 & $2 \mathrm{be} e^{ \pm} p$ & 22 & $19.1 \pm 2.2$ & 0 & $e^{ \pm}{ }_{\mathrm{j} \tau} \mp$ & 386 & $418 \pm 18.9$ & 0 \\
\hline $4 \mathrm{j} \mu^{ \pm} \mu^{\mp}$ & 38 & $48.4 \pm 6.2$ & 0 & $2 \mathrm{be} e_{\mathrm{j} p}$ & 19 & $19.4 \pm 2.2$ & 0 & $e^{ \pm} \mathrm{j} \tau^{ \pm}$ & 160 & $162.8 \pm 3.5$ & 0 \\
\hline $4 \mathrm{j} \mu^{ \pm}$ & 1363 & $1350.1 \pm 37.7$ & 0 & $2 \mathrm{be} e_{\mathrm{j}}^{ \pm}$ & 63 & $63 \pm 3.4$ & 0 & $e^{ \pm} \mathrm{j} p \tau^{\mp}$ & 48 & $44.6 \pm 3.3$ & 0 \\
\hline $3 \mathrm{j}$ high $-\Sigma p_{T}$ & 159926 & $159143 \pm 1061.9$ & $\begin{array}{l}0 \\
0\end{array}$ & $2 \mathrm{be} e^{ \pm}$ & 96 & $92.1 \pm 4.1$ & 0 & $e^{ \pm} \mathrm{j} p \tau^{ \pm}$ & 11 & $8.3 \pm 1.5$ & 0 \\
\hline $\begin{array}{l}3 \mathrm{j} l o w-\Sigma p_{T} \\
3 \mathrm{j} 2 \gamma\end{array}$ & $\begin{array}{r}62681 \\
151\end{array}$ & $\begin{array}{r}64213.1 \pm 496 \\
177.5 \pm 7.1\end{array}$ & $\begin{array}{l}0 \\
0\end{array}$ & $\tau^{ \pm} \tau^{\mp}$ & 856 & $872.5 \pm 19$ & 0 & $e^{ \pm} \mathrm{j} p$ & 121431 & $121023 \pm 747.6$ & 0 \\
\hline $3 \mathrm{j} \tau^{ \pm}$high $-\Sigma p_{T}$ & 68 & $76.9 \pm 3$ & 0 & $\gamma \not p$ & 3793 & $3770.7 \pm 127.3$ & 0 & $e^{ \pm} \mathrm{j} \gamma p$ & 159 & $192.6 \pm 10.9$ & 0 \\
\hline $3 \mathrm{j} p$ high $-\Sigma p_{T}$ & 1706 & $1899.4 \pm 77.6$ & 0 & $\mu^{ \pm}$ & 381 & $440.9 \pm 7.3$ & 0 & $e^{ \pm} \mathrm{j} \gamma$ & 1389 & $1368.9 \pm 38.9$ & 0 \\
\hline $3 \mathrm{j} p$ low- $\Sigma p_{T}$ & 42 & $36.2 \pm 5.7$ & 0 & $\mu^{ \pm}$ & 60 & $75.7 \pm 3.4$ & 0 & $e^{ \pm} \mathrm{j} \mu^{\mp} \not p$ & 42 & $33 \pm 2.9$ & 0 \\
\hline $3 \mathrm{j} \gamma \tau^{ \pm}$ & 39 & $37.8 \pm 3.6$ & 0 & $\mu^{ \pm}$ & 15 & $12 \pm 2$ & 0 & $e^{ \pm} \mu^{ \pm} \not p$ & 16 & $9.2 \pm 1.9$ & 0 \\
\hline $3 \mathrm{j} \gamma p$ & 204 & $249.8 \pm 24.4$ & 0 & $\mu^{ \pm} \not p$ & 734290 & $734296 \pm 4897.8$ & 0 & $e^{ \pm} \mathrm{j} \mu^{\mp}$ & 62 & $63.8 \pm 3.2$ & 0 \\
\hline $3 \mathrm{j} \gamma$ & 24639 & $24899.4 \pm 372.4$ & 0 & $\mu^{ \pm}$ & 475 & $469.8 \pm 12.5$ & 0 & $e^{ \pm} \mathrm{j} \mu^{ \pm}$ & 13 & $8.2 \pm 2$ & 0 \\
\hline $3 \mathrm{j} \mu^{ \pm} p$ & 2884 & $2971.5 \pm 52.1$ & 0 & $\mu^{ \pm}$ & 169 & $198.5 \pm 8.2$ & 0 & $e^{ \pm} e^{\mp} 4 \mathrm{j}$ & 148 & $159.1 \pm 7$ & 0 \\
\hline $3 \mathrm{j} \mu^{ \pm} \gamma p$ & 10 & $3.6 \pm 1.9$ & 0 & $\mu^{ \pm}$ & 83 & $60 \pm 3.1$ & 0 & $e^{ \pm} e^{\mp} 3 \mathrm{j}$ & 717 & $743.6 \pm 24.4$ & 0 \\
\hline $3 \mathrm{j} \mu^{ \pm} \gamma$ & 15 & $7.9 \pm 2.9$ & 0 & $\mu^{ \pm} \mu^{\prime}$ & 25283 & $25178.5 \pm 86.5$ & 0 & $e^{ \pm} e^{\mp} 2 \mathrm{j} p$ & 32 & $41.4 \pm 5.6$ & 0 \\
\hline $3 \mathrm{j} \mu^{ \pm} \mu^{\mp}$ & 175 & $177.8 \pm 16.2$ & 0 & $\mathrm{j} 2 \gamma \not p$ & 36 & $30.4 \pm 4.2$ & 0 & $e^{ \pm} e^{\mp} 2 \mathrm{j} \gamma$ & 10 & $11.4 \pm 2.9$ & 0 \\
\hline $3 \mathrm{j} \mu^{ \pm}$ & 5032 & $4989.5 \pm 108.9$ & 0 & $\mathrm{j} 2 \gamma$ & 1822 & $1813.2 \pm 27.4$ & 0 & $e^{ \pm} e^{\mp} 2 \mathrm{j}$ & 3638 & $3566.8 \pm 72$ & 0 \\
\hline $3 \mathrm{~b} 2 \mathrm{j}$ & 23 & $28.9 \pm 4.7$ & 0 & $\mathrm{j} \tau^{ \pm}$high- $\Sigma p_{T}$ & 52 & $56.2 \pm 2.5$ & 0 & $e^{ \pm} e^{\mp} \tau^{ \pm}$ & 18 & $16.1 \pm 1.7$ & 0 \\
\hline $3 \mathrm{bj}$ & 82 & $82.6 \pm 5.7$ & 0 & $\mathrm{j} \tau^{ \pm} \tau^{\mp}$ & 203 & $252.2 \pm 8.7$ & 0 & $e^{ \pm} e^{\mp \not p}$ & 822 & $831.8 \pm 13.6$ & 0 \\
\hline $3 b_{+}$ & 67 & $85.6 \pm 7.7$ & 0 & $\mathrm{j} p p$ high $-\Sigma p_{T}$ & 4432 & $4431.7 \pm 45.2$ & 0 & $e^{ \pm} e^{\mp} \gamma$ & 191 & $221.9 \pm 5.1$ & 0 \\
\hline $2 \tau^{ \pm}$ & 498 & $512.7 \pm 14.2$ & 0 & $\mathrm{j} \gamma \tau^{ \pm}$ & 526 & $476 \pm 9.3$ & 0 & $e^{ \pm} e^{\mp} \mathrm{j} p$ & 155 & $170.8 \pm 12.4$ & \\
\hline $\begin{array}{l}2 \gamma \not p \\
2 \gamma\end{array}$ & $\begin{array}{r}128 \\
5548\end{array}$ & $\begin{array}{c}107.2 \pm 6.9 \\
5562.8 \pm 40.5\end{array}$ & $\begin{array}{l}0 \\
0\end{array}$ & $\mathrm{j} \gamma \not p$ & $\begin{array}{r}1882 \\
103319\end{array}$ & $\begin{array}{c}1791.9 \pm 72.3 \\
102124+570.6\end{array}$ & $\begin{array}{l}0 \\
0\end{array}$ & $e^{ \pm} e^{\mp} \mathrm{j} \gamma$ & 48 & $45 \pm 3.9$ & \\
\hline $2 \mathrm{j}$ high $-\Sigma p_{T}$ & 190773 & $190842 \pm 781.2$ & 0 & $\mathrm{j}_{\mu} \pm \tau_{\tau} \mp$ & $\begin{array}{r}103319 \\
71\end{array}$ & $\begin{array}{c}102124 \pm 570.6 \\
98+3.9\end{array}$ & $\begin{array}{l}0 \\
0\end{array}$ & $e^{ \pm} e^{\mp} \mathrm{j}$ & 17903 & $18258.2 \pm 204.4$ & \\
\hline $2 \mathrm{j}$ low- $-\Sigma p_{T}$ & 165984 & $162530 \pm 1581$ & 0 & $j \mu \pm \tau$ & $\begin{array}{l}11 \\
15\end{array}$ & $\begin{array}{l}90 \pm 3.9 \\
12 \pm 2\end{array}$ & 0 & $e^{ \pm} e^{\mp}$ & 98901 & $99086.9 \pm 147.8$ & \\
\hline $2 \mathrm{j} 2 \tau^{ \pm}$ & 22 & $40.6 \pm 3.2$ & 0 & $\begin{array}{l}\mathrm{j} \mu \\
\mathrm{i} \mu \tau^{-} \mp\end{array}$ & 15 & $\begin{array}{c}12 \pm 2 \\
308+26\end{array}$ & 0 & $\mathrm{~b} 6 \mathrm{j}$ & 51 & $42.3 \pm 3.8$ & \\
\hline $2 \mathrm{j} 2 \gamma \not p$ & 11 & $8 \pm 2.4$ & 0 & & 26 & $30.8 \pm 2.6$ & U & b5j & 237 & $192.5 \pm 7.1$ & 0 \\
\hline $2 \mathrm{j} 2 \gamma$ & 580 & $581 \pm 13.7$ & 0 & & 109081 & $108323 \pm 707.7$ & 0 & b4j high- $\Sigma p_{T}$ & 26 & $23.4 \pm 2.6$ & \\
\hline $2 \mathrm{j} \tau^{ \pm}$high $-\Sigma p_{T}$ & 96 & $114.6 \pm 3.3$ & 0 & $\mathrm{j} \mu^{ \pm} \gamma p$ & 171 & $171.1 \pm 31$ & 0 & b4j low $-\Sigma p_{T}$ & 836 & $821.7 \pm 15.9$ & \\
\hline & & & & $\mathrm{j} \mu^{ \pm} \gamma$ & 152 & $190 \pm 39.3$ & 0 & b3j high- $\Sigma p_{T}$ & 12081 & $12071 \pm 84.1$ & \\
\hline & & & & & & & & b3j 1 & 2974 & $2873 \pm 31$ & \\
\hline
\end{tabular}


the Tevatron, observation in the $H \rightarrow b \bar{b}$ decay channel will be extremely difficult at the LHC. Given the importance of observing the Higgs boson in its main decay mode, searches at the Tevatron have thus to be viewed as complementary to, rather than in competition with, the Higgs boson search at the LHC. Failure to observe the Higgs boson in the mass range considered would also be a very important result, as it would indicate a breakdown of the standard model and give directions for alternative theories.

Current electroweak data point to the existence of a light Higgs, which means that the so-far elusive Higgs particle is within reach of Tevatron. Soon the LHC will also exploit its potential for discovery of new particles. However, a light Higgs boson corresponds to the less favorable scenario at LHC for an early discovery. In addition, it may take some time to operate the ATLAS and CMS detectors and to understand the new data. At the Tevatron, a Higgs boson in this mass range can only be convincingly observed if the integrated luminosity delivered is sufficiently large $\left(8-9 \mathrm{fb}^{-1}\right)$ and the current analyses continue to improve their sensitivity. Furthermore, if its mass is heavier than $\approx 140 \mathrm{GeV}$, the MSSM will be ruled out, a conclusion which applies to the majority of supersymmetric models.

This review has summarized the searches for Higgs bosons and beyond-the-standard-model physics at the Tevatron that have been conducted until May 2008. However, despite all efforts, no significant deviations from the standard model predictions have been found to date based on data samples corresponding to integrated luminosities of up to $2.5 \mathrm{fb}^{-1}$. Of course, this should not be taken to mean absence of new physics in these data, for there are still a number of $\mathrm{fb}^{-1}$ of frontier physics ahead of us at the Tevatron.

\section{Acknowledgments}

I would like to thank my colleagues both at Fermilab and at other collaborating institutions, especially those who operate the Tevatron accelerator and construct, maintain, and calibrate the CDF and D $\varnothing$ detectors, essential for any physics analysis reported here.

I wish to thank all the colleagues for providing valuable input and/or proofreading this document, in particular I would like to address special thanks to Chris Hays, Gavin Davies, Gregorio Bernardi, and Matthew Herndon. I am also grateful to Todd Adams, Volker Büscher, Dmitri Denisov, Monica D'onofrio, Wade Fisher, Aurelio Juste, Stefan Söldner-Rembold, Patrice Verdier, and Darien Wood for their suggestions and comments during the preparation of this review.

\section{References}

1. P. W. Higgs, Phys. Lett. 12, 132 (1964).

2. C. Muñoz, Int. J. Mod. Phys. A19, 3093 (2004).

3. For reviews on supersymmetric theories, see J. Wess and B. Zumino, Nucl. Phys. B70, 39 (1974); P. Fayet and S. Ferrara, Phys. Rep. 32, 249 (1977); H.P. Nilles, Phys. Rep. 110, 1 (1984).
4. H.E. Haber and G.L. Kane, Phys. Rep. 117, 75 (1985).

5. H.P. Nilles, Phys. Rep. 110, 1 (1984).

6. CDF Collaboration, CDF physics results page: http://www-cdf.fnal.gov/physics/physics.html

7. $\mathrm{D} \varnothing$ Collaboration, $D \varnothing$ physics results page: http://www-d0.fnal.gov/Run2Physics/WWW/results.htm

8. CDF Collaboration, Phys. Rev. D71, 032001 (2005); J. Phys. G34, 2457 (2007).

9. DØ Collaboration, Nucl. Instrum. Methods A565, 463 (2006).

10. CDF Collaboration, Nucl. Instrum. Methods A447, 1 (2000); ibid., 453, 84 (2000).

11. CDF Collaboration, Nucl. Instrum. Methods A526, 249 (2004).

12. CDF Collaboration, Nucl. Instrum. Methods A566, 375 (2006), and references therein.

13. G.C. Blazey et al., in Proceedings of the Workshop: "QCD and Weak Boson Physics in Run II," edited by U. Baur, R.K. Ellis, and D.Zeppenfeld (Fermilab, Batavia, IL, 2000), p. 47; see Sec. 3.5 for details.

14. A. Djouadi, The anatomy of electro-weak symmetry breaking. I: The Higgs boson in the standard model, arXiv:hep-ph/0503172v2 (2005).

15. C. F. Kolda, H. Murayama, J. High Energy Phys. 0007, 035 (2000).

16. $\mathrm{CDF}$ and $\mathrm{D} \varnothing$ Collaborations, Combination of $C D F$ and $D \varnothing$ results on the mass of the top quark, CDF Note 9225, DØ Note 5626 (2008).

17. LEP Collaborations and LEP Electroweak Working Group, Precision electroweak measurements and constraints on the standard model, arXiv:hepex/0712.0929v2 (2007).

18. CDF Collaboration, Phys. Rev. Lett. 99, 151801 (2007) and CDF Collaboration, First Run II measurement of the $W$ boson mass, submitted to Phys. Rev. D, arXiv:hep-ex/0708.3642v1 (2007).

19. LEP Electroweak Working Group, http://lepewwg.web.cern.ch/LEPEWWG/

20. LEP Collaborations, Phys. Lett. B565, 61 (2003).

21. U. Aglietti et al., Tevatron-for-LHC Report: Higgs, arXiv:hep-ph/0612172v2 (2007).

22. T. Han, G. Valencia, S. Willenbrock, Phys. Rev. Lett. 69, 3274 (1992); E.L. Berger and J. Campbell, Phys. Rev. D70, 073011 (2004); T. Figy, C. Oleari, D. Zeppenfeld, Phys. Rev. D68, 073005 (2003).

23. W. Beenakker et al., Phys. Rev. Lett. 87, 201805 (2001); L. Reina and S. Dawson, Phys. Rev. Lett. 87, 201804 (2001); S. Dawson, L.H. Orr, L. Reina, D. Wackeroth, Phys. Rev. D67, 071503 (2003).

24. R.V. Harlander and W.B. Kilgore, Phys. Rev. D68, 013001 (2003); J. Campbell, R.K. Ellis, F. Maltoni, S. Willenbrock, Phys. Rev. D67, 095002 (2003); S. Dawson, C.B. Jackson, L. Reina, D. Wackeroth, Phys. Rev. Lett. 94, 031802 (2005); S. Dittmaier, M. Krämer, M. Spira, Phys. Rev. D70, 074010 (2004); S. Dawson, C.B. Jackson, L. Reina, D. Wackeroth, Phys. Rev. D69, 074027 (2004).

25. K.A. Assamagan et al., The Higgs Working Group: Summary Report 2003, arXiv:hep-ph/0406152v1 (2004).

26. O. Brein, A. Djouadi, R. Harlander, Phys. Lett. B579, 149 (2004); M.L. Ciccolini, S. Dittmaier, M. Krämer, Phys. Rev. D68, 073003 (2003). 
27. D. Graudenz, M. Spira, P.M. Zerwas, Phys. Rev. Lett. 70, 1372 (1993); S. Catani, D. de Florian, M. Grazzini, P. Nason, J. High Energy Phys. 0307, 028 (2003); R.V. Harlander and W.B. Kilgore, Phys. Rev. Lett. 88, 201801 (2002); C. Anastasiou and K. Melnikov, Nucl. Phys. B646, 220 (2002); C. Anastasiou, K. Melnikov, F. Petriello, Phys. Rev. Lett. 93, 262002 (2004); U. Aglietti, R. Bonciani, G. Degrassi, A. Vicini, Phys. Lett. B595, 432 (2004); G. Degrassi and F. Maltoni, Phys. Lett. B600, 255 (2004).

28. T. Sjöstrand et al., Comput. Phys. Commun. 135, 238 (2001); T. Sjöstrand, L. Lonnblad, S. Mrenna, PYTHIA 6.2: Physics and manual, arXiv:hepph/0108264v1 (2001).

29. H.L. Lai et al., Eur. Phys. J. C12, 375 (2000); Phys. Rev. D55, 1280 (1997).

30. A. Djouadi, J. Kalinowski, M. Spira, Comput. Phys. Commun. 108, 56 (1998); arXiv:hep-ph/9704448 (1997).

31. M.L. Mangano, M. Moretti, F. Piccinini, R. Pittau, A.D. Polosa, J. High Energy Phys. 0307, 001 (2003); arXiv:hep-ph/0206293v2 (2003).

32. S. Frixione and B.R. Webber, J. High Energy Phys. 06, 029 (2002); arXiv:hep-ph/0204244v2 (2002).

33. G. Corcella, I.G. Knowles, G. Marchesini, S. Moretti, K. Odagiri, P. Richardson, M.H. Seymour, B.R. Webber, J. High Energy Phys. 0101, 010 (2001); arXiv:hep-ph/0011363v3 (2002).

34. A. Pukhov, E. Boos, M. Dubinin, V. Edneral, V. Ilyin, D. Kovalenko, A. Kryukov, V. Savrin, S. Shichanin, A. Semenov, CompHEP: A package for evaluation of Feynman diagrams and integration over multi-particle phase space. User's manual for version 33, arXiv:hepph/9908288v2 (2000).

35. J. Campbell and R.K. Ellis, Phys. Rev. D60, 113006 (1999), arXiv:hep-ph/9905386v2 (1999).

36. T. Scanlon, FERMILAB-THESIS-2006-43.

37. L. Breiman et al., Classification and Regression Trees, Wadsworth (1984); Y. Freund and R.E. Shapire, Experiments with a new boosting algorithm, in Machine Learning: Proceedings of the Thirteenth International Conference, pp. 148-156 (1996); DØ Collaboration, Phys. Rev. Lett. 98, 181802 (2007).

38. CDF Collaboration, Strong evidence of $Z Z$ production in $p \bar{p}$ collisions at $\sqrt{s}=1.96 \mathrm{Te} V$, accepted to Phys. Rev. Lett., arXiv:hep-ex/0801.4806v1 (2008).

39. DØ Note $5620, Z Z \rightarrow \ell \ell \nu \bar{\nu}$ production in $p \bar{p}$ collisions at $\sqrt{s}=1.96 \mathrm{TeV},(2008)$.

40. CDF Collaboration, Phys. Rev. Lett. 100, 041801 (2008).

41. D $\varnothing$ Collaboration, A combined search for the standard model Higgs boson at $\sqrt{s}=1.96 \mathrm{TeV}$, Phys. Lett. B663, 26 (2008).

42. CDF Note 9219, Search for standard model Higgs boson production in association with $W^{ \pm}$boson at $C D F$ with $1.9 \mathrm{fb}^{-1},(2008)$.

43. DØ Note 5472, Search for WH Production using a neural network approach in $p \bar{p}$ Collisions at $\sqrt{s}=$ 1.96 TeV with $1.7 \mathrm{fb}^{-1}$ of Data, (2007).

44. DØ Collaboration, Phys. Rev. Lett. 97, 161803 (2006).

45. CDF Collaboration, Search for the Higgs boson in events with missing transverse energy and $b$ quark jets produced in proton-antiproton collisions at $\sqrt{s}=$ $1.96 \mathrm{TeV}$, submitted to Phys. Rev. Lett., arXiv:hepex/0802.0432v1 (2008).
46. DØ Note 5586, Search for the standard model Higgs boson in the $\mathrm{HZ} \rightarrow b b \nu \nu$ channel in $2.1 \mathrm{fb}^{-1}$ of $p \bar{p}$ collisions at $\sqrt{s}=1.96 \mathrm{TeV},(2008)$.

47. CDF Note 9166, Neural network search for standard model Higgs boson in $\not_{T}$ plus jets channel with $1.7 \mathrm{fb}^{-1},(2008)$.

48. CDF Note 8742, Search for $Z H \rightarrow \ell \ell b \bar{b}$ in $1 \mathrm{fb}^{-1}$ of CDF Run 2 data, (2007).

49. DØ Note 5482, Search for $Z H \rightarrow \ell \ell b \bar{b}$ in $p \bar{p}$ collisions at $\sqrt{s}=1960 \mathrm{GeV},(2007)$.

50. DØ Collaboration, Phys. Lett. B655, 209 (2007).

51. H.E. Haber, G.L. Kane, T. Sterling, Nucl. Phys. B161, 493 (1979); J.F. Gunion, R. Vega, J. Wudka, Phys. Rev. D42, 1673 (1990); J.L. Basdevant, E.L. Berger, D. Dicus, C. Kao, S. Willenbrock, Phys. Lett. B313, 40 (1993); V. Barger, N.G. Deshpande, J.L. Hewett, T.G. Rizzo,arXiv:hepph/9211234 (1992); P. Bamert and Z. Kunszt, Phys. Lett. B306, 335 (1993); A.G. Akeroyd, Phys. Lett. B368, 89 (1996); M.C. Gonzalez-Garcia, S.M. Lietti, S.F. Novaes, Phys. Rev. D57, 7045 (1998); A. Barroso, L. Brucher, R. Santos, Phys. Rev. D60, 035005 (1999); L. Brucher and R. Santos, Eur. Phys. J. C12, 87 (2000).

52. B. Dobrescu, Phys. Rev. D63, 015004 (2001); B. Dobrescu, G. Landsberg, K. Matchev, FERMILABPUB99/324-T.

53. L. Hall and C. Kolda, Phys. Lett. B459, 213 (1999); H. Cheng, B.A. Dobrescu, C.T. Hill, Nucl. Phys. B589, 249 (2000).

54. D $\varnothing$ Collaboration, Phys. Rev. Lett. 97, 151804 (2006).

55. CDF Note 7307, Search for the Wh production using high-p ${ }_{T}$ isolated like-sign dilepton events in Run II with $1.9 \mathrm{fb}^{-1}$, (2008).

56. DØ Note 5485, Search for the Associated Higgs Boson Production $p \bar{p} \rightarrow W H \rightarrow W W W^{(*)} \rightarrow \ell^{ \pm} \ell^{ \pm},(2007)$.

57. CDF Collaboration, Phys. Rev. Lett. 97, 081802 (2006).

58. DØ Collaboration, Phys. Rev. Lett. 96, 011801 (2006).

59. CDF Note 9236 , Search for Higgs to $W W^{*}$ production using a combined matrix element and neural network technique at Tevatron using $2.4 \mathrm{fb}^{-1}$ of data, (2008).

60. DØ Note 5537, Search for the Higgs Boson in $H \rightarrow W W^{*} \rightarrow l l^{\prime}\left(l, l^{\prime}=e, m u\right)$ decays with $1.7 \mathrm{fb}^{-1}$ of Data at DØ, (2007).

61. DØ Note 5624, Search for the Higgs boson in $H \rightarrow W W^{*} \rightarrow l l^{\prime}\left(l, l^{\prime}=e, m u\right)$ decays with 1.2 $\mathrm{fb}^{-1}$ at DØ in Run IIb, (2008).

62. DØ Collaboration, Search for $h_{f} \rightarrow \gamma \gamma$ with the DØ detector at $\sqrt{s}=1.96 \mathrm{TeV}$, submitted to Phys. Rev. Lett., arXiv:hep-ex/0803.1514v12008.

63. DØ Note 5601, Search for a light Higgs boson in $\gamma \gamma$ final states at DØ, (2008).

64. CDF Note 9248, Search for the SM Higgs Boson using $\tau$ lepton. Simultaneous Search for $W H / Z H / V B F / g g H$ in 2t's+2jets event, (2008).

65. Tevatron New Phenomena \& Higgs Working Group (TEVNPHWG), Combined CDF and DØ upper limits on standard model Higgs boson production, CDF Note 8961, DØ Note 5536, FERMILAB-PUB-07-656E (2007), arXiv:hep-ex/0712.2383v1.

66. Tevatron New Phenomena \& Higgs Working Group (TEVNPHWG), Combined CDF and DO upper limits on standard model Higgs boson production with 
up to $2.4 \mathrm{fb}^{-1}$ of data, CDF Note 9290, DØ Note 5645, FERMILAB-PUB-08-069-E (2008); arXiv:hepex/0804.3423v1 (21 April 2008).

67. CDF Note 8941, Combined upper limits on standard model Higgs boson production, (2007).

68. DØ Note 5625, Combined upper limits on standard model Higgs boson production from the DØ experiment in 1.0-2.3 $\mathrm{fb}^{-1},(2008)$.

69. T. Junk, Nucl. Instrum. Methods A434, 435 (1999); A. Read, in "First Workshop on Confidence Limits", CERN Report No. CERN-2000-005 (2000).

70. W. Fisher, FERMILAB-TM-2386-E (2007).

71. J.R Espinosa and M. Quiros, Phys. Lett. B266, 389 (1991); R. Hempfling and A. Hoang, Phys. Lett. B331, 99 (1994); J.A Casa, J. Espinosa, M. Quiros and A. Riotto, Nucl. Phys. B436, 3 (1995); (E) B439, 466 (1995); M. Carena, J. Espinosa, M. Quiros, C.E.M. Wagner, Phys. Lett. B355, 209 (1995); M. Carena, M. Quiros, C.E.M. Wagner, Nucl. Phys. B461, 407 (1996); S. Heinemeyer, W. Hollik, G. Weiglein, Phys. Rev. D58, 091701 (1998), Phys. Lett. B440, 296 (1998), J. High Energy Phys. 0006, 009 (2000); J.R Espinosa and R.J. Zhang, Nucl. Phys. B586, 3 (2000); A. Brignole, G. Degrassi, P. Slavich, F. Zwirner, Nucl. Phys. B631, 195 (2002), Nucl. Phys. B643, 79 (2002); S. Heinemeyer, W. Hollik, H. Rzehak, G. Weiglein, Eur. Phys. J. C39, 465 (2005).

72. S. Heinemeyer, W. Hollik, G. Weiglein, Eur. Phys. J. C9, 343 (1999), Comput. Phys. Commun. 124, 76 (2000); G. Degrassi, S. Heinemeyer, W. Hollik, P. Slavich, G. Weiglein, Eur. Phys. J. C28, 133 (2003); M. Frank et al., J. High Energy Phys. 0702, 047 (2007).

73. M. Carena and H.E. Haber,Prog. Part. Nucl. Phys. 50, 63 (2003).

74. S. Bertolini, F. Borzumati, A. Masiero, G. Ridolfi, Nucl. Phys. B353, 591 (1991); K.S. Babu and C. F. Kolda, Phys. Rev. Lett. 84, 228 (2000); C. Hamzaoui, M. Pospelov, M. Toharia, Phys. Rev. D59, 095005 (1999); G. Isidori and A. Retico, J. High Energy Phys. 0111, 001 (2001); A.J. Buras, P.H. Chankowski, J. Rosiek, L. Slawianowska, Phys. Lett. B546, 96 (2002); A.J. Buras, P.H. Chankowski, J. Rosiek, L. Slawianowska, Nucl. Phys. B659, 3 (2003); A. Dedes and A. Pilaftsis, Phys. Rev. D67, 015012 (2003); G. D'Ambrosio, G.F. Giudice, G. Isidori, A. Strumia, Nucl. Phys. B645, 155 (2002); M. Dugan, B. Grinstein, L.J. Hall, Nucl. Phys. B255, 413 (1985); J. Foster, K.I. Okumura, L. Roszkowski, J. High Energy Phys. 0508, 094 (2005).

75. M. Carena, S. Heinemeyer, C. E. M. Wagner, and G. Weiglein, Eur. Phys. J. C26, 601-607 (2003).

76. J.R. Ellis, S. Heinemeyer, K.A. Olive, A.M. Weber, G. Weiglein, J. High Energy Phys. 0708, 083 (2007).

77. ALEPH, DELPHI, L3, and OPAL Collaborations, The LEP Working Group for Higgs Bosons Searches, Eur. Phys. J. C47, 547 (2006).

78. O. Buchmueller et al., Phys. Lett. B657, 87 (2007).

79. J. Campbell, R. K. Ellis, F. Maltoni, S. Willenbrock, Phys. Rev. D67, 095002 (2003).

80. S. Dawson, C. B. Jackson, L. Reina, D. Wackeroth, Mod. Phys. Lett. A21, 89 (2006).

81. DØ Collaboration, Phys. Rev. Lett. 95, 151801 (2005).
82. DØ Note 5503, Search for neutral Higgs bosons at high $\tan \beta$ in multijet events, (2006).

83. CDF Note 8954, Search for Higgs bosons produced in association with b-quarks, (2007).

84. CDF Note 9284, Search for Higgs bosons produced in association with b-quarks, (2008).

85. DØ Collaboration, Search for neutral Higgs bosons in multi-b-jet events in $p \bar{p}$ collisions at $\sqrt{s}=$ $1.96 \mathrm{TeV}$, submitted to Phys. Rev. Lett., arXiv:hepex/0805.3556v1 (2008).

86. M. Carena, S. Heinemeyer, C.E.M. Wagner, G. Weiglein, Eur. Phys. J. C45, 797 (2006).

87. M. Spira, HIGLU: A program for the calculation of the total Higgs production cross section at hadron colliders via gluon fusion including QCD corrections, arXiv:hep-ph/9510347v1 (1995).

88. CDF Collaboration, Phys. Rev. Lett. 96, 011802 (2006).

89. D $\varnothing$ Collaboration, Phys. Rev. Lett. 97, 121802 (2006).

90. CDF Note 9071, Search for neutral MSSM Higgs bosons decaying to tau pairs with $1.8 \mathrm{fb}^{-1}$ of data, (2007).

91. DØ Collaboration, Search for Higgs bosons decaying to tau pairs in $p \bar{p}$ collisions with the DØ detector, submitted to Phys. Rev. Lett., arXiv:hepex/0805.2491v1 (2008).

92. LEP Higgs Working Group, Note 2004-01 (2004).

93. CDF Collaboration, Phys. Rev. Lett. 96, 042003 (2006).

94. CDF Note 8353, Search for anomalous tau production in b-tagged top quark events, (2006).

95. G. Senjanovic and R.N. Mohapatra, Phys. Rev. D12, 1502 (1975).

96. G. B. Gelmini and M. Roncadelli, Phys. Lett. B99, 411 (1981).

97. N. Arkani-Hamed, A.G. Cohen, E. Katz, A.E. Nelson, J. High Energy Phys. 0207, 034 (2002).

98. CDF Collaboration, Phys. Rev. Lett. 93, 221802 (2004).

99. DØ Collaboration, Phys. Rev. Lett. 93, 141801 (2004).

100. CDF Note 8050, Search for $p \bar{p} \rightarrow H^{++} H^{--} \rightarrow \ell^{+} \tau^{+} \ell^{-} \tau^{-}$, (2006).

101. D $\varnothing$ Collaboration, Search for pair production of doubly-charged Higgs bosons in the $H^{++} H^{--} \rightarrow \mu^{+} \mu^{+} \mu^{-} \mu^{-}$final state at $D \varnothing$, submitted to Phys. Rev. Lett., arXiv:hep-ex/0803.1534v1 (2008).

102. OPAL Collaboration, Phys. Lett. B526, 221 (2002); OPAL Collaboration, Phys. Lett. B295, 347 (1992); L3 Collaboration, Phys. Lett. B576, 18 (2003); DELPHI Collaboration, Phys. Lett. B552, 127 (2003).

103. CDF Collaboration, Phys. Rev. Lett. 95, 071801 (2005).

104. DØ Note 5067, Search for fermiophobic Higgs boson in $3 \gamma+X$ events, (2007).

105. S. Weinberg, Phys. Rev. D13, 974 (1976); L. Susskind, Phys. Rev. D20, 2619 (1979).

106. D $\varnothing$ Collaboration, Phys. Rev. Lett. 98, 221801 (2007).

107. CDF Note 9302, Search for technicolor particles produced in association with $W^{ \pm}$boson with $1.9 \mathrm{fb}^{-1}$ at CDF, (2008). 
108. DØ Collaboration, Phys. Rev. Lett. 95, 151805 (2005).

109. CDF Collaboration, Phys. Rev. D77, 052002 (2008).

110. CDF Collaboration, Phys. Rev. Lett. 98, 221803 (2007).

111. CDF Collaboration, Phys. Rev. Lett. 99, 191806 (2007).

112. D $\varnothing$ Note 5464, Search for the associated production of chargino and neutralino in final states with two electrons and an additional lepton, (2007).

113. CDF Note 9176, A unified search for associated production of chargino-neutralino at CDF using leptons, (2008).

114. LEP SUSY Working Group, ibid., note LEPSUSYWG/01-03.1.

115. H. Baer, F.E. Paige, S.D. Protopescu, X. Tata, ISAJET : A monte carlo event generator for $p p$, $\bar{p} p$, and $e^{+} e^{-}$reactions, see for instance arXiv:hepex/0312045v1.

116. W. Beenakker, M. Krämer, T. Plehn, M. Spira, P.M. Zerwas, Nucl. Phys. B515, 3 (1998).

117. DØ Collaboration, Phys. Lett. B660, 449 (2008).

118. CDF Note 9229, Search for gluino and squark production in multijets plus missing $E_{T}$ final State, (2008).

119. J. Pumplin, D.R. Stump, J. Huston, H.L. Lai, P. Nadolsky, W.K. Tung, J. High Energy Phys. 0207, 012 (2002); D. Stump, J. Huston, J. Pumplin, W.K. Tung, H.L. Lai, S. Kuhlmann, J.F. Owens, J. High Energy Phys. 0310, 046 (2003).

120. LEP SUSY Working Group, ibid., note LEPSUSYWG/02-06.2.

121. DØ Note 5468, Search for squark production in events with jets, hadronically decaying taus and missing transverse energy with the DØ detector at $\sqrt{s}=$ $1.96 \mathrm{TeV}$ in the Run IIa data, (2007).

122. DØ Collaboration, Search for scalar top quarks in the acoplanar charm jets and missing transverse energy final state in $p \bar{p}$ collisions at $\sqrt{s}=1.96 \mathrm{TeV}$, accepted by Phys. Lett. B, arXiv:hep-ex/0803.2263v1 (2008).

123. DØ Collaboration, Phys. Lett. B645, 119 (2007).

124. CDF Collaboration, Phys. Rev. D76, 072010 (2007).

125. DØ Collaboration, Phys. Rev. Lett. 97, 171806 (2006).

126. CDF Collaboration, Phys. Rev. Lett. 96, 171802 (2006).

127. CDF Note 9332, Search of gluino-mediated sbottom production in the MET+b-jet sample, (2008).

128. D $\varnothing$ Note 5438, Search for scalar top admixture in the $\bar{t}$ lepton+jets final state at $\sqrt{s}=1.96 \mathrm{TeV}$ in $1 \mathrm{fb}^{-1}$ of DØ Data, (2007).

129. DØ Collaboration, Phys. Lett. B659, 500 (2008).

130. DØ Note 5598, Search for pair production of the supersymmetric partner of the top quark in the $\tilde{t}_{1} \tilde{t}_{1} \rightarrow b \bar{b} e^{ \pm} \mu^{ \pm} \tilde{\nu} \overline{\tilde{\nu}}$ decay channel at DØ, (2008).

131. J. Feng and T. Moroi, Phys. Rev. D58, 035001 (1998).

132. DØ Note 4746, A search for charged massive stable particles at $D \varnothing,(2005)$.

133. J. Gunion and S. Mrenna, Phys. Rev. D62, 015002 (2000).

134. CDF Collaboration, Phys. Rev. Lett. 99, 121801 (2007); Search for heavy, long-lived neutralinos that decay to photons at CDF II using photon timing, submitted to Phys. Rev. D, arXiv:hep-ex/0804.1043v1 (2008).
135. CDF Collaboration, Phys. Rev. D71, 031104 (2005).

136. D $\varnothing$ Collaboration, Phys. Rev. Lett. 94, 041801 (2005).

137. V. Buescher et al., for the CDF and DØ Collaborations, Combination of CDF and DØ limits on a gauge mediated SUSY model using diphoton and missing transverse energy channel, arXiv:hepex/0504004v12005.

138. B.C. Allanach et al., Eur. Phys. J. C25, 113 (2002).

139. DØ Collaboration, Phys. Lett. B659, 856 (2008).

140. CDF Collaboration, Phys. Rev. Lett. 98, 131804 (2007).

141. DØ Collaboration, Phys. Lett. B638, 441 (2006).

142. CDF Note 7835, Search for pair production of scalar top quarks decaying to a $\tau$ lepton and a $b$ quark, (2005).

143. CDF Collaboration, Phys. Rev. Lett. 96, 211802 (2006).

144. D $\varnothing$ Collaboration, Search for sneutrino particles in $e+m u$ final states in $p \bar{p}$ collisions at $\sqrt{s}=$ $1.96 \mathrm{TeV}$., submitted to Phys. Rev. Lett., arXiv:hepex/0711.3207v2 (2007).

145. CDF Collaboration, Phys. Rev. Lett. 95, 252001 (2005).

146. CDF Collaboration, Phys. Rev. Lett. 95, 131801 (2005).

147. DØ Collaboration, Phys. Rev. Lett. 97, 111801 (2006).

148. M. Byrne, C. Kolda, P. Regan, Phys. Rev. D66, 075007 (2002).

149. M.J. Strassler and K.M. Zurek, Echoes of a hidden valley at hadron colliders, arXiv:hep-ph/0604261 (2006).

150. NuTeV Collaboration, Phys. Rev. Lett. 87, 041801 (2001).

151. D $\varnothing$ Collaboration, Phys. Rev. Lett. 97, 161802 (2006).

152. A. De Rujula, S. L. Glashow, and U. Sarid, Nucl. Phys. B333, 173 (1990).

153. CDF Note 8701, Search for charged massive stable particles, (2007).

154. H. Frampton, P.Q. Hung, M. Sher, Phys. Rep. 330, 263 (2000).

155. CDF Note 7244, Search for long-lived parents of the $Z$ boson, (2004).

156. DØ Note 5454, Search for long-lived particles decaying into $Z$ bosons, (2007).

157. N. Arkani-Hamed, S. Dimopoulos, G.F. Giudice, A. Romanino, Nucl. Phys. B709, 3 (2005).

158. A. Arvanitaki, S. Dimopoulos, A. Pierce, S. Rajendran, J. Wacker, Stopping gluinos, arXiv:hep$\mathrm{ph} / 05062422005$.

159. DØ Collaboration, Phys. Rev. Lett. 99, 131801 (2007).

160. W. Buchmuller and D. Wyler, Phys. Lett. B177, 377 (1986).

161. H. Georgi and S.L. Glashow, Phys. Rev. Lett. 32, 438 (1974).

162. J.L. Hewett and T.G. Rizzo, Phys. Rep. 183, 193 (1989).

163. B. Schrempp and F. Schrempp, Phys. Lett. B153, 101 (1985).

164. CDF Collaboration, Phys. Rev. D72, 051107 (2005).

165. CDF Collaboration, Phys. Rev. D71, 112001 (2005), Erratum-ibid., D71, 119901 (2005). 
166. DØ Collaboration, Phys. Rev. D71, 071104 (2005).

167. DØ Collaboration, Phys. Lett. B640, 230 (2006).

168. CDF Note 9329, Search for new physics in the exclusive dijet plus missing $E_{T}$ event sample, (2008).

169. CDF Collaboration, Phys. Rev. D73, 051101 (2006).

170. DØ Collaboration, Phys. Lett. B636, 183 (2006).

171. DØ Note 5370, Search for second generation scalar leptoquarks in the $\mu \nu j j$ final state with the DØ detector, (2007).

172. DØ Note 5447, Search for third generation scalar leptoquarks using the $\tau b \tau b$ final state, (2007).

173. DØ Collaboration, Phys. Rev. Lett. 99, 061801 (2007).

174. CDF Collaboration, Search for third generation vector leptoquarks in $p \bar{p}$ collisions at $\sqrt{s}=$ $1.96 \mathrm{TeV}$, submitted to Phys. Rev. D, arXiv:hepex/0706.2832v1 (2007).

175. D $\varnothing$ Collaboration, Phys. Lett. B647, 74 (2007).

176. H. Terazawa, M. Yasue, K. Akama, M. Hayashi, Phys. Lett. B112, 387 (1982); F.M. Renard, Nuovo. Cimento. A77, 1 (1983); A. De Rujula, L. Maiani, R. Petronzio, Phys. Lett. B140, 253 (1984); E.J. Eichten, K.D. Lane, M.E. Peskin, Phys. Rev. Lett. 50, 811 (1983).

177. D $\varnothing$ Collaboration, Phys. Rev. D77, 091102(R) (2008).

178. CDF Collaboration, Phys. Rev. Lett. 94, 101802 (2005).

179. CDF Collaboration, Phys. Rev. Lett. 97, 191802 (2006).

180. DØ Collaboration, Phys. Rev. D73, 111102 (2006).

181. U. Baur, M. Spira, P. Zerwas, Phys. Rev. D42, 8158 (1990).

182. DØ Collaboration, Phys. Rev. D74, 011104 (2006).

183. CDF Note 9246, Search for new particles decaying to dijets in $p \bar{p}$ collisions at $\sqrt{s}=1.96 \mathrm{TeV},(2008)$.

184. L. Randall and R. Sundrum, Phys. Rev. Lett. 83, 3370 (1999); ibid., 83, 4690 (1999).

185. K.D. Lane and M.V. Ramana, Phys. Rev. D44, 2678 (1991); E. Eichten and K.D. Lane, Phys. Lett. B327, 129 (1994); K. Lane and S. Mrenna, Phys. Rev. D67, 115011 (2003).

186. J. Bagger, C. Schmidt, S. King, Phys. Rev. D37, 1188 (1988); P.H. Frampton and S.L. Glashow, Phys. Lett. B190, 157 (1987).

187. CDF Collaboration, Phys. Rev. D76, 072006 (2007).

188. CDF Note 9164, A search for massive gluon decaying to top pair in lepton+jet channel, (2008).

189. CDF Note 9234, Search for Heavy Top $t^{\prime} \rightarrow W q$ in lepton plus jets events, (2008).

190. P. Langacker, R. W. Robinett, J.L. Rosner, Phys. Rev. D30, 1470 (1984).

191. J.C. Pati and A. Salam, Phys. Rev. D10, 275 (1974); R.N. Mohapatra and J.C. Pati, Phys. Rev. D11, 566 (1975), 11, 2558 (1975); G. Senjanovic and R.N. Mohapatra, Phys. Rev. D12, 1502 (1975).

192. CDF Note 9160, High-mass di-electron resonance search in $p \bar{p}$ collisions at $\sqrt{s}=1.96 \mathrm{TeV},(2008)$.

193. CDF Collaboration, Phys. Rev. Lett. 96, 211801 (2006).

194. CDF Collaboration, Phys. Rev. Lett. 99, 171802 (2007).

195. DØ Note 4375, Search for heavy $Z^{\prime}$ bosons in the dielectron channel with $200 \mathrm{pb}^{-1}$ of data with the $D \varnothing$ detector, (2004).
196. E. Eichten, K. Lane, J. Womersley, Phys. Lett. B405, 305 (1997).

197. DØ Note 4561, Search for technicolor particles $\rho_{T}$ and $\omega_{T}$ in the dielectron channel with $200 \mathrm{pb}^{-1}$ of data with the DØ detector, (2004).

198. D $\varnothing$ Note 4577, Search for heavy $Z^{\prime}$ bosons in the dimuon channel with 250 pb-1 of data with the DØ detector, (2004).

199. B. Murakami, Phys. Rev. D65, 055003 (2002).

200. P. Langacker and J. Kang, Phys. Rev. D71, 035014 (2005).

201. DØ Collaboration, Search for $t \bar{t}$ resonances in the lepton+jets final state in $p \bar{p}$ collisions at $\sqrt{(s)}=$ $1.96 \mathrm{TeV}$, submitted to Phys. Lett. B, arXiv:hepex/0804.3664v1 (2008).

202. DØ Note 5600, Search for $t \bar{t}$ resonances in the lepton+jets final state in $p \bar{p}$ collisions at $\sqrt{(s)}=$ $1.96 \mathrm{TeV},(2008)$.

203. C.T. Hill and S. Parke, Phys. Rev. D49, 4454 (1994).

204. CDF Note 8087, Search for resonant $t \bar{t}$ production in $p \bar{p}$ collisions at $\sqrt{(s)}=1.96 \mathrm{TeV},(2008)$.

205. CDF Note 8675 , Limit on resonant $t \bar{t}$ production in $p \bar{p}$ collisions at $\sqrt{(s)}=1.96 \mathrm{TeV},(2008)$.

206. CDF Collaboration, Phys. Rev. D75, 091101 (2007).

207. D $\varnothing$ Collaboration, Phys. Rev. Lett. 100, 031804 (2008).

208. DØ Collaboration, Phys. Lett. B641, 423 (2006).

209. DØ Collaboration, Search for $W^{\prime}$ boson resonances decaying to a top quark and a bottom quark, accepted by Phys. Rev. Lett., arXiv:hepex/0803.3256v1 (2008).

210. CDF Note $9150, W^{\prime}$-like resonances in the $t \bar{b}$ decay channel with $1.9 \mathrm{fb}^{-1}$, (2008).

211. N. Arkani-Hamed, S. Dimopoulos, G. Dvali, Phys. Lett. B429, 263 (1998).

212. DØ Collaboration, Phys. Rev. Lett. 95, 091801 (2005).

213. CDF Collaboration, Phys. Rev. Lett. 99, 171801 (2007).

214. DØ Collaboration, Phys. Rev. Lett. 100, 091802 (2008).

215. CDF Collaboration, Search for new heavy particles decaying to $Z Z \rightarrow$ eeee in $p \bar{p}$ collisions at $\sqrt{s}=$ $1.96 \mathrm{TeV}$, submitted to Phys. Rev. D, arXiv:hepex/0801.1129v1 (2008).

216. G. Giudice, R. Rattazzi, J.D. Wells, Nucl. Phys. B544, 3 (1999).

217. D $\varnothing$ Collaboration, Search for large extra dimensions in the mono-photon final state at $\sqrt{s}=$ $1.96 \mathrm{TeV}$, submitted to Phys. Rev. Lett., arXiv:hepex/0803.2137v1 (2008).

218. CDF Collaboration, Phys. Rev. Lett. 97, 171802 (2006).

219. CDF Note 9308, Search for new physics in the exclusive jets/photon+MET channel in Run-II, (2008).

220. DØ Note 4400, Search for large extra spatial dimensions in jets + missing $E_{T}$ topologies, (2004).

221. D $\varnothing$ Collaboration, Search for large extra dimensions in the mono-photon final state at $\sqrt{s}=$ $1.96 \mathrm{TeV}$, submitted to Phys. Rev. Lett., arXiv:hepex/0803.2137v1 (2008).

222. CDF Note XXXX, Search for anomalous diphoton $+X$ production, (2007).

223. CDF Note 9339, Search for anomalous $\gamma \gamma+\not_{T}$ events in $2 \mathrm{fb}^{-1}$ of data, (2007). 
224. CDF Collaboration, Phys. Rev. Lett. 97, 031801 (2006).

225. CDF Collaboration, Phys. Rev. D75, 112001 (2007).

226. CDF Note 9335, Search for new physics in lepton + photon + missing transverse energy + b-jets events and $t \bar{t} \gamma$ cross section measurement, (2008).

227. CDF Note 8452, Searching for the anomalous production of $Z$-Bosons with high transverse momentum in $0.94 \mathrm{fb}^{-1}$ at the Tevatron, (2006).

228. CDF Collaboration, Phys. Rev. Lett. 89, 041802 (2002).

229. CDF preliminary result, Vista/Sleuth global search for new physics in $2 \mathrm{fb}^{-1}$ of $p \bar{p}$ collisions at $\sqrt{s}=$ $1.96 \mathrm{TeV}$ (2008); see C. Henderson, for the CDF Collaboration, Contribution to the proceedings of the XLIIIth Rencontres de Moriond: QCD and High-Energy Interactions, arXiv:hep-ex/0805.0742v1 (2008).

230. CDF Collaboration, Model-independent global search for new high-pT physics at CDF, submitted to Phys. Rev. Lett., arXiv:hep-ex/0712.2534v1 (2007).

231. F. Maltoni and T. Stelzer, J. High Energy Phys. 0302, 027 (2003)

232. CDF Collaboration, Model-independent and quasimodel-independent search for new physics at $C D F$, submitted to Phys. Rev. D, arXiv:hepex/0712.1311v1 (2007). 